\title{
Measurement of the Top Quark Pair Production Cross Section in the Dilepton Channel Using \\ Lepton+Track Selection
}

\author{
Robert Emil Wagner
}

\author{
A Dissertation Presented To The \\ Faculty Of Princeton University \\ In Candidacy For The Degree Of \\ Doctor of Philosophy
}

Recommended For Acceptance

By The Department Of Physics

Advisor: Chris Tully

November 2008 
(C) by Robert Emil Wagner, 2008. All rights reserved. 


\begin{abstract}
The production cross section for t $\bar{t}$ pairs decaying into two lepton final states was measured using data from the D $\varnothing$ detector at Fermilab. The measurement was made using a lepton+track selection, where one lepton is fully identified and the second lepton is observed as an isolated track. This analysis is designed to complement similar studies using two fully identified leptons [1]. The cross section for the lepton+track selection was found to be

$$
\sigma=5.2_{-1.4}^{+1.6}(\text { stat })_{-0.8}^{+0.9} \text { (syst) } \pm 0.3 \text { (lumi) pb }
$$
\end{abstract}

The combined cross section using both the lepton+track data and the data from the electron+electron, electron+muon, and muon+muon samples is:

$$
\sigma=6.4_{-0.9}^{+0.9}(\text { stat })_{-0.7}^{+0.8} \text { (syst) } \pm 0.4 \text { (lumi) } \mathrm{pb}
$$




\section{Acknowledgments}

I would first like to thank the entire $\mathrm{D} \varnothing$ collaboration, without whom none of this work would have been possible. I would also like to thank my advisor, Chris Tully, for his help and support, as well as the rest of the Princeton University physics faculty. I also want to acknowledge the many helpful conversations I have had with Elizaveta Shabalina, Christian Schwanenberger, Frederic Deliot, Peter Renkel, Michael Begel, and Joe Haley. 


\section{Contents}

Abstract $\quad$ iii

Acknowledgments $\quad$ iv

1 Introduction 1

1.1 Overview of Dilepton Cross Section Measurements . . . . . . . . . . 1

1.2 Motivation for a Lepton + Track Selection . . . . . . . . . . . . 2

2 Standard Model $\quad 6$

3 The Tevatron and the DØ Detector $\quad 11$

3.1 Fermilab and the Tevatron Accelerator . . . . . . . . . . . . . . 11

3.2 DØ Detector . . . . . . . . . . . . . . . . . . 14

3.2.1 Coordinates ........................ 15

3.3 Tracking . . . . . . . . . . . . . . . . 16

3.4 Preshower ............................ 20

3.4.1 Calorimeter ..................... . . 21

3.4 .2 Muon System . . . . . . . . . . . . . . . . . . 26

3.5 Trigger System . . . . . . . . . . . . . . . . . . . 29

3.5.1 Example: Electron trigger . . . . . . . . . . . . . 31

4 Object Identification $\quad 36$

4.1 Electrons and Photons . . . . . . . . . . . . . . . . 36

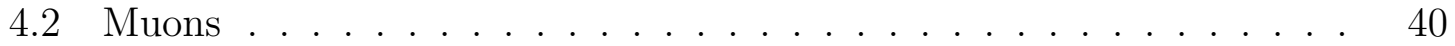

4.3 Tracks . . . . . . . . . . . . . . . . . . . 43

4.4 Jets . . . . . . . . . . . . . . . . . 46

4.4.1 Upgraded Level 1 Confirmation Algorithm . . . . . . . . . . . 48

4.5 Missing Transverse Momentum _. . . . . . . . . . . . . . 53 


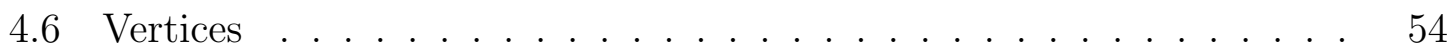

5 Event Selection $\quad 56$

5.1 Electron + Track Selection . . . . . . . . . . . . . 56

5.2 Muon + Track Selection . . . . . . . . . . . . . . . 57

5.3 Further Z background Rejection . . . . . . . . . . . . . . . . . . 59

5.4 Tagged samples . . . . . . . . . . . . . . . . . . . 59

6 Event Samples $\quad 63$

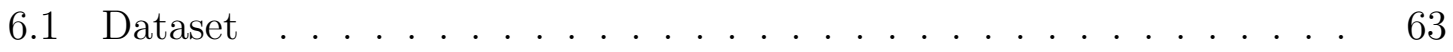

6.2 Signal Simulation . . . . . . . . . . . . . . . . 63

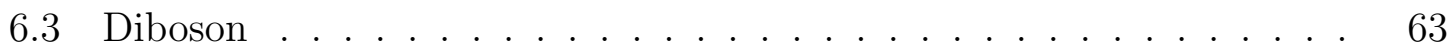

$6.4 \mathrm{Z}$ boson . . . . . . . . . . . . . . . . 63

$6.5 \mathrm{Z} \rightarrow \tau \tau \ldots \ldots \ldots \ldots \ldots \ldots$

6.6 Fake Lepton Background Estimation . . . . . . . . . . . . . . . 67

$\begin{array}{llr}7 & \text { Systematics } & 69\end{array}$

$\begin{array}{llr}8 & \text { Results } & 72\end{array}$

8.1 Lepton + Track Cross Section . . . . . . . . . . . . . . . . . 72

8.2 Combined Dilepton Cross Section . . . . . . . . . . . . 76

8.3 Conclusion and Outlook . . . . . . . . . . . . . . 78

$\begin{array}{ll}\text { A Track Identification Scale Factors } & 80\end{array}$

$\begin{array}{ll}\text { B Details of the Matrix Method } & 91\end{array}$

C Efficiencies for the Matrix Method Calculation $\quad 95$

$\begin{array}{ll}\text { D Control Plots } & 101\end{array}$

$\begin{array}{ll}\text { References } & 115\end{array}$ 


\section{List of Figures}

1 Diagram of the $\mathrm{D} \varnothing$ detector, showing the cryostat wall in the calorimeter and the reduced muon coverage on the bottom of the detector. . .

2 An event display for an example lepton+track event. Thee left-hand side shows an $\mathrm{r}-\mathrm{z}$ view while the right-hand side is an $\mathrm{r}-\phi$ view. Colored bars represent the energy deposited in the calorimeter, where red is electromagnetic energy and blue is hadronic energy, and tracks reconstructed by the central tracker are shown. Colored rectangles outside the calorimeter display represent hits in the muon system. The indicated electron is the electron which was identified by its isolated

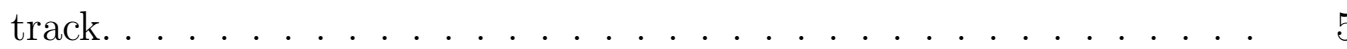

$3 \quad \mathrm{t} \overline{\mathrm{t}}$ pair production diagrams . . . . . . . . . . . . . . . . 7

4 Theoretical predicted cross section versus center-of-mass collision energy for various physical processes. The collision energies for the Tevatron and the LHC are indicated. . . . . . . . . . . . . . . . . . 10

5 Diagram of the Tevatron accelerator . . . . . . . . . . . . . . 12

6 Diagram of the $\mathrm{D} \emptyset$ detector. . . . . . . . . . . . . . 15

$7 \quad$ Diagram of the DØ Silicon Microstrip Tracker . . . . . . . . . . . 17

8 Layout of the readout cells in the DØ calorimeter. . . . . . . . . . . 22

9 Cutaway diagram of the $\mathrm{D} \varnothing$ calorimeter. . . . . . . . . . . 23

10 Diagram of a unit cell in the $\mathrm{D} \emptyset$ calorimeter. . . . . . . . . . . 24

11 Diagram of (a) the muon system scintillators and (b) the muon drift chambers. . . . . . . . . . . . . . . . . . . . . . 28

12 Trigger rates as a function of time for Store 3224 . . . . . . . . . . . 31

13 Definitions of the variables used for Level 1 electron trigger decisions. Each variable is defined as the black squares divided by the sum of the black and grey squares. . . . . . . . . . . . . . . . . 
14 Definitions of the variables used for the new Level 2 likelihood variable. Each variable is defined as the black squares divided by the sum of the black and grey squares, except for the variable NSratio which is defined as black divided by grey. . . . . . . . . . . . . . . .

15 Turn on curves for the E1 trigger suite for various cuts on the Level 2 likelihood variable. .................. . . . . 35

16 Turn on curves for the E2 trigger suite for various cuts on the Level 2 likelihood variable. . . . . . . . . . . . . . . .

17 Number of towers above threshold using the updated Level 1 confirmation algorithm. . . . . . . . . . . . . . . .

18 Comparison of the original and updated Level 1 confirmation algorithms. The original algorithm is the black curve and the updated algorithm is the blue curve. . . . . . . . . . . . . . 52

19 Comparison of various Level 1 confirmation algorithms. The original algorithm is the black curve and the implemented algorithm is the blue curve (these are the same as in Figure 18). The red curve shows the results of the additional corrections discussed in the text. . . . . . .

$20 \quad E_{T}^{Z-f i t}$ distribution (in $\mathrm{GeV}$ ) for $\mathrm{t} \overline{\mathrm{t}}$ and $\mathrm{Z} \rightarrow \mathrm{ll}$ samples. All selection cuts have been applied except for $\not_{T}, \quad Z_{T}^{Z-f i t}$ and b-tagging. Top row is the electron+track channel and bottom row is the muon+track channel. . . . . . . . . . . . . . . . . .

21 Distribution of the output variable from the DØ Neural Network tagger. (a) is the electron+track one jet bin, (b) is the electron+track two jet bin, (c) is the muon+track one jet bin, and (d) is the muon+track two jet bin. . . . . . . . . . . . . . . . . . . 
22 Electron+track mass distributions $\left(\left(E_{e}+E_{t}\right)^{2}-\left(\vec{p}_{e}+\vec{p}_{t}\right)^{2}\right.$, where $E_{e}$ and $\vec{p}_{e}$ are the energy and momentum of the electron and $E_{t}$ and $\vec{p}_{t}$ are the energy and momentum of the track) in $\mathrm{GeV}$ for the low $\not_{T}$ sample used for $\mathrm{Z}$ boson $\mathrm{K}$ factor determination. (a) is the one jet bin and (b) is the two jet bin. The $K_{Z}$ factor has been applied in these plots, such that the Monte Carlo distribution is normalized to the data. 65

23 Muon+track mass distributions $\left(\left(E_{\mu}+E_{t}\right)^{2}-\left(\vec{p}_{\mu}+\vec{p}_{t}\right)^{2}\right.$, where $E_{\mu}$ and $\vec{p}_{\mu}$ are the energy and momentum of the muon and $E_{t}$ and $\vec{p}_{t}$ are the energy and momentum of the track) in $\mathrm{GeV}$ for the low $\not_{T}$ sample used for $\mathrm{Z}$ boson $\mathrm{K}$ factor determination. (a) is the one jet bin and (b) is the two jet bin. The $K_{Z}$ factor has been applied in these plots, such that the Monte Carlo distribution is normalized to the data. . . . . .

24 Lepton+track mass distributions $\left(\left(E_{l e p}+E_{t}\right)^{2}-\left(\vec{p}_{l e p}+\vec{p}_{t}\right)^{2}\right.$, where $E_{l e p}$ and $\vec{p}_{l e p}$ are the energy and momentum of the lepton and $E_{t}$ and $\vec{p}_{t}$ are the energy and momentum of the track) in GeV for the low $E_{T}$ tagged sample used to determine the heavy flavor $\mathrm{K}$ factor. (a) is the electron+track channel one jet bin, (b) is the electron+track channel two jet bin, (c) is the muon+track one jet bin, and (d) is the muon+track channel two jet bin. All K factors have been applied for

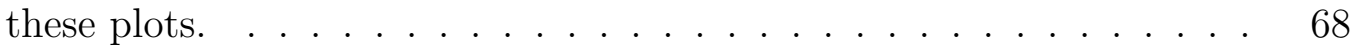

25 Measured cross section vs. assumed mass of the top quark. . . . . . 75

26 Cross sections for the individual dilepton channels, the dilepton combination, and the lepton+jets channel for an assumed top mass of $175 \mathrm{GeV}$. The inner error bars are the systematic error (including the luminosity systematic), and the outer error bars are the total error (statistical plus systematic). . . . . . . . . . . . . 
27 Monte Carlo to data scale factor of the track in the electron+track channel as a function of $p_{T}$ for (a) the $\Delta z$ (lepton vertex, track) cut, (b) the distance-from-vertex significance cut, (c) the $\chi^{2}$ cut, (d) the loose track isolation cut, (e) all loose cuts combined, and (f) the tight track isolation cut. . . . . . . . . . . . . . . . .

28 Monte Carlo to data scale factor of the track in the electron+track channel as a function of $\eta$ for (a) the $\Delta z$ (lepton vertex, track) cut, (b) the distance-from-vertex significance cut, (c) the $\chi^{2}$ cut, (d) the loose track isolation cut, (e) all loose cuts combined, and (f) the tight track isolation cut

29 Monte Carlo to data scale factor of the track in the electron+track channel as a function of number of jets for (a) all loose cuts combined, and (b) the tight track isolation cut. . . . . . . . . . . .

30 2D representation of Monte Carlo to data scale factor in the electron+track channel for (a) all loose cuts combined, and (b) the tight track isolation cut.

31 Monte Carlo to data scale factor of the track in the muon+track channel as a function of $p_{T}$ for (a) the $\Delta \mathrm{z}$ (lepton vertex, track) cut, (b) the distance-from-vertex significance cut, (c) the $\chi^{2}$ cut, (d) the loose track isolation cut, (e) all loose cuts combined, and (f) the tight track isolation cut. . . . . . . . . . . . . . . . .

32 Monte Carlo to data scale factor of the track in the muon+track channel as a function of $\eta$ for (a) the $\Delta$ z(lepton vertex, track) cut, (b) the distance-from-vertex significance cut, (c) the $\chi^{2}$ cut, (d) the loose track isolation cut, (e) all loose cuts combined, and (f) the tight track isolation cut. . . . . . . . . . . . . . . . . . 
33 Monte Carlo to data scale factor of the track in the muon+track channel as a function of number of jets for (a) all loose cuts combined, and (b) the tight track isolation cut. . . . . . . . . . . . . . .

34 2D representation of Monte Carlo to data scale factor in the muon+track channel for (a) all loose cuts combined, and (b) the tight track isolation

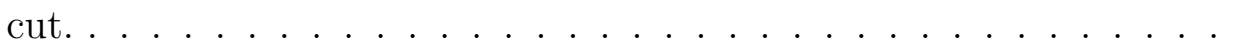

35 Closure test in the electron+track channel for (a) loose track scale factor as a function of $p_{T}$ (b) tight track scale factor as a function of $p_{T}$ (c) loose track scale factor as a function of $\eta(\mathrm{d})$ tight scale track factor as a function of $\eta \ldots \ldots \ldots \ldots$

36 Closure test in the muon+track channel for (a) loose track scale factor as a function of $p_{T}$ (b) tight track scale factor as a function of $p_{T}$ (c) loose track scale factor as a function of $\eta(d)$ tight track scale factor as a function of $\eta$. . . . . . . . . . . . . . . . . . . .

$37 \epsilon_{s i g}^{e}$ in the electron+track channel vs $\not_{T}$ for $(\mathrm{a})$ the 1 jet bin and (b) the 2 jet inclusive bin. . . . . . . . . . . . . . .

$38 \epsilon_{s i g}^{t r k}$ in the electron+track channel vs $\not_{T}$ for (a) the 1 jet bin and (b) the 2 jet inclusive bin. . . . . . . . . . . . . . . . . 96

$39 \epsilon_{s i g}^{\mu}$ in the muon+track channel vs $\not_{T}$ for (a) the 1 jet bin and (b) the 2 jet inclusive bin. . . . . . . . . . . . . . . . 96

$40 \epsilon_{s i g}^{t r k}$ in the muon+track channel vs $\#_{T}$ for (a) the 1 jet bin and (b) the 2 jet inclusive bin . . . . . . . . . . . . . . .

$41 \epsilon_{b k g}^{e}$ in the electron+track channel vs $\not_{T}$ for (a) the 1 jet bin and (b) the 2 jet inclusive bin. Only data points up to $\not_{T}$ of $15 \mathrm{GeV}$ are used for the efficiency calculation. The quadratic fit is used for systematic error estimation. 
$42 \epsilon_{b k g}^{t r k}$ in the electron+track channel vs $\not_{T}$ for (a) the 1 jet bin and (b) the 2 jet inclusive bin. Only data points up to $\not_{T}$ of $15 \mathrm{GeV}$ are used for the efficiency calculation. The quadratic fit is used for systematic

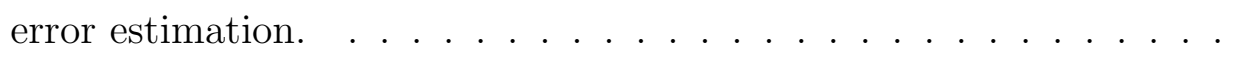

$43 \epsilon_{b k g}^{\mu}$ in the muon+track channel vs $E_{T}$ for (a) the 1 jet bin and (b) the 2 jet inclusive bin. Only data points up to $\not_{T}$ of $15 \mathrm{GeV}$ are used for the efficiency calculation. The quadratic fit is used for systematic error estimation. . . . . . . . . . . . . . .

$44 \epsilon_{b k g}^{t r k}$ in the muon+track channel vs $\#_{T}$ for (a) the 1 jet bin and (b) the 2 jet inclusive bin. Only data points up to $\not_{T}$ of $15 \mathrm{GeV}$ are used for the efficiency calculation. The quadratic fit is used for systematic error estimation. . . . . . . . . . . . . . . .

$45 \epsilon_{b k g}^{t r k}$ in the electron+track channel vs instantaneous luminosity for (a) the 1 jet bin and (b) the 2 jet inclusive bin. The linear fit is used for systematic error estimation. . . . . . . . . . . . . . . . 100

$46 \epsilon_{b k g}^{t r k}$ in the muon+track channel vs instantaneous luminosity for (a) the 1 jet bin and (b) the 2 jet inclusive bin. The linear fit is used for systematic error estimation. . . . . . . . . . . . . . . . . . 100

47 Mass (in GeV) of electron-track pair in electron+track. . . . . . . . . 101

$48 \quad E_{T}($ in $\mathrm{GeV})$ in electron+track. . . . . . . . . . . . . . 102

$49 \quad E_{T}^{Z-f i t}($ in $\mathrm{GeV})$ in electron+track. . . . . . . . . . . . . . . 102

50 Transverse mass (in $\mathrm{GeV}$ ) of electron and $Z_{T}$ in electron+track. . . 102

51 Transverse mass (in GeV) of track and $Z_{T}$ in electron+track. . . . 103

$52 \Delta \phi$ of electron and $E_{T}$ in electron+track. . . . . . . . . 103

$53 \Delta \phi$ of track and $Z_{T}$ in electron+track. . . . . . . . . 103

$54 \mathrm{Z} p_{T}$ (vector sum of electron $p_{T}$ and track $p_{T}$, in $\mathrm{GeV}$ ) in electron+track.104

55 Electron $p_{T}($ in $\mathrm{GeV})$ in electron+track. . . . . . . . . . . . . . 104 
Electron $\eta$ in electron+track. . . . . . . . . . . . . . . . . 104

57 Electron $\phi$ in electron+track. . . . . . . . . . . . . . . . . . 105

58 Track $p_{T}($ in $\mathrm{GeV})$ in electron+track. . . . . . . . . . . . 105

59 Track $\eta$ in electron+track. . . . . . . . . . . . . . . . . . 105

60 Track $\phi$ in electron + track. . . . . . . . . . . . . . . . . . . 106

61 Leading jet $p_{T}$ (in $\mathrm{GeV}$ ) in electron+track. . . . . . . . . . . . 106

62 Leading jet $\eta$ in electron+track. . . . . . . . . . . . . . . . 106

63 Leading jet $\phi$ in electron+track. . . . . . . . . . . . . . . . . 107

64 Second leading jet $p_{T}$ (in $\mathrm{GeV}$ ) in electron+track. . . . . . . . . 107

65 Second leading jet $\eta$ in electron+track. . . . . . . . . . . . 107

66 Second leading jet $\phi$ in electron+track. . . . . . . . . . . . . . 108

67 Mass (in $\mathrm{GeV}$ ) of muon-track pair in muon+track. . . . . . . . . . . 108

$68 E_{T}($ in $\mathrm{GeV})$ in muon+track. . . . . . . . . . . . . . . 108

$69 \quad E_{T}^{Z-f i t}($ in $\mathrm{GeV})$ in muon+track. . . . . . . . . . . . . . 109

70 Transverse mass (in $\mathrm{GeV}$ ) of muon and $E_{T}$ in muon+track. . . . . 109

71 Transverse mass (in $\mathrm{GeV}$ ) of track and $\mathbb{E}_{T}$ in muon+track. . . . . 109

$72 \Delta \phi$ of muon and $\not_{T}$ in muon+track. . . . . . . . . . 110

$73 \Delta \phi$ of track and $z_{T}$ in muon+track. . . . . . . . . . . . 110

$74 \mathrm{Z} p_{T}$ (vector sum of muon $p_{T}$ and track $p_{T}$, in $\mathrm{GeV}$ ) in muon+track. 110

75 Muon $p_{T}($ in $\mathrm{GeV})$ in muon+track. . . . . . . . . . . . . . 111

76 Muon $\eta$ in muon+track. . . . . . . . . . . . . . . . . . . . . 111

77 Muon $\phi$ in muon+track. . . . . . . . . . . . . . . . . . . . . . 111

78 Track $p_{T}$ (in $\left.\mathrm{GeV}\right)$ in muon+track. . . . . . . . . . . . . . 112

79 Track $\eta$ in muon+track. . . . . . . . . . . . . . . . . . . . . . . . . 112

80 Track $\phi$ in muon+track. . . . . . . . . . . . . . . . . . . . . . . . 112

81 Leading jet $p_{T}$ (in $\mathrm{GeV}$ ) in muon+track. . . . . . . . . . . 113

82 Leading jet $\eta$ in muon+track. . . . . . . . . . . . . . . . 113 
83 Leading jet $\phi$ in muon+track. . . . . . . . . . . . . . . . . 113

84 Second leading jet $p_{T}($ in $\mathrm{GeV})$ in muon+track. . . . . . . . . . . . . 114

85 Second leading jet $\eta$ in muon+track. . . . . . . . . . . . . . . 114

86 Second leading jet $\phi$ in muon+track. . . . . . . . . . . . . . . . 114 


\section{List of Tables}

1 Parameters of the Tevatron. . . . . . . . . . . . . . . . 14

2 Trigger requirements at Level 1 and Level 2 for the E1 and E2 trigger suites. The Level 1 isolation fraction and EM fraction variables are defined in Figure 13. The Level 2 isolation fraction is defined as the EM energy of the seed tower plus the highest neighboring tower, divided by the total energy (EM plus hadronic) in a 3x3 grid around the seed

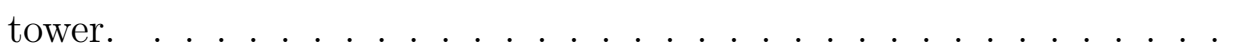

3 Expected trigger rate for the E1 and E2 trigger suites for various cuts on the Level 2 likelihood variable. . . . . . . . . . . . . . . . . 34

4 Threshold setting used for the updated Level 1 Confirmation algorithm. 49

5 Efficiency to pass $E_{T}^{Z-f i t}$ cut in the electron+track channel. All numbers are before b-tagging has been applied. Number of events in the first column is the number of events after $E_{T}$ has been applied but before $E_{T}^{Z-f i t} \ldots \ldots \ldots \ldots \ldots \ldots \ldots \ldots \ldots \ldots \ldots \ldots \ldots \ldots$

6 Efficiency to pass $\#_{T}^{Z-f i t}$ cut in the muon+track channel. All numbers are before b-tagging has been applied. Number of events in the first column is the number of events after $\not_{T}$ has been applied but before $E_{T}^{Z-f i t} \ldots \ldots \ldots \ldots \ldots \ldots \ldots \ldots \ldots \ldots \ldots \ldots \ldots \ldots$

7 Number of expected and observed events in the $\mathrm{Z}$ sample in the electron+track channel, used to calculate $K_{Z}$. . . . . . . . . . . . 64

8 Number of expected and observed events in the $\mathrm{Z}$ sample in the muon+track channel, used to calculate $K_{Z} \ldots \ldots \ldots \ldots \ldots \ldots$

9 Number of expected and observed events in the b-tagged Z sample in the electron+track channel. These results are used to calculate a relative heavy flavor to light flavor $\mathrm{K}$ factor. $\mathrm{Z}+$ heavy flavor and $\mathrm{Z}+$ light flavor include contributions from both $\mathrm{Z} \rightarrow$ ee and $\mathrm{Z} \rightarrow \tau \tau$. . 
10 Number of expected and observed events in the b-tagged Z sample in the muon+track channel. These results are used to calculate a relative heavy flavor to light flavor $\mathrm{K}$ factor. $\mathrm{Z}+$ heavy flavor and $\mathrm{Z}+$ light flavor include contributions from both $\mathrm{Z} \rightarrow \mu \mu$ and $\mathrm{Z} \rightarrow \tau \tau$. . . . . . .

11 Table of systematic uncertainties in the electron+track, muon+track, and combined channels. . . . . . . . . . . . . . . . 73

12 Number of predicted and observed events. t $\bar{t}$ estimate based on a 5.0 pb cross section. . . . . . . . . . . . . . . . 73

13 Inputs to the cross section calculation. . . . . . . . . . . . . 74

14 Inputs to the cross section calculation from the lepton+track channels. The listed branching ratio and efficiency is given relative to t $\overline{\mathrm{t}}$ decays to all dilepton final states ee, $\mathrm{e} \mu, \mathrm{e} \tau, \mu \mu, \mu \tau$, and $\tau \tau$. . . . . . . . . 77

15 Inputs to the cross section calculation from the fully identified dilepton channels. The given branching fraction and efficiency is relative to $t \overline{\mathrm{t}}$ decay to the listed final state (ee, $\mathrm{e} \mu, \mu \mu)$, either by direct decay of the $\mathrm{W}(W \rightarrow l \nu)$ or through a $\tau$ intermediary $(W \rightarrow \tau \nu \rightarrow l \nu \nu) . \ldots$

16 Table of signal and fake rates used in the matrix method background estimate. . . . . . . . . . . . . . . . . . 93

17 Number of events in the loose and tight samples used for background estimation in electron+track and muon+track channels. . . . . . . . .

18 Number of real and fake events expected in the tight lepton, tight track sample with their statistical errors. . . . . . . . . . . . . . 


\section{Introduction}

The Tevatron machine is located at the Fermilab facility in Batavia, Illinois. It is presently the highest energy particle collider in the world and will remain so until the start-up of the Large Hadron Collider at the European Center for Nuclear Research (CERN) in Geneva in late 2008 or 2009.

The Tevatron is a circular accelerator which collides protons with their antimatter counterparts, the antiproton. Superconducting electromagnets are used to guide the particle beams around the ring. As they have opposite electric charge, the protons and antiprotons circulate in opposite directions. The proton beams and antiproton beams collide at two locations on the ring, the CDF and $\mathrm{D} \varnothing$ detectors. The data for this experiment was taken with the $\mathrm{D} \varnothing$ detector from April 2002 to March 2006.

Top quarks are typically produced in pairs in the Tevatron collider. The top quark nearly always decays into a bottom quark and a $\mathrm{W}$ boson. The $\mathrm{W}$ boson can decay either hadronically (two quarks) or leptonically (a charged lepton and a neutrino). Thus, the signature of a tét pair depends on the decay of the two W's: there is the all jets channel where both W's decay hadronically, the lepton+jets channel where one decays hadronically and the other leptonically, and finally there is the dilepton channel where both $\mathrm{W}$ bosons decay into leptons. This analysis studies the cross section of dilepton final states.

\subsection{Overview of Dilepton Cross Section Measurements}

This analysis focuses on the dilepton channel where the $\mathrm{W}$ bosons decayed leptonically. Final states which contain $\tau$ leptons can be very difficult to identify as the $\tau$ decays too rapidly to be directly observed and it must be seen through its decay products. The $\tau$ can either decay into hadrons, which can resemble a 
hadronic jet, or into a ligher lepton (either an electron or muon) plus neutrinos. As the $\tau$ particle can be difficult to distinguish from other particles, it is typical for dilepton analyses to focus only on final states without $\tau$ leptons, namely, the electron+electron, electron+muon, and muon+muon final states. Searches in these channels are sensitive to decays which involve intermediary $\tau$ particles, i.e., where the original $\mathrm{W}$ decays to a $\tau$, and this $\tau$ decays into a lighter charged lepton.

Of the three possible final states of interest (electron+electron, electron+muon, and muon+muon), the electron+electron and muon+muon final states suffer from substantial backgrounds from $\mathrm{Z} \rightarrow$ ee and $\mathrm{Z} \rightarrow \mu \mu$ decays. Cuts must be imposed to remove this background, which leads to substantial signal loss. Also, low lepton identification efficiency reduces the yield in dilepton analyses. In particular, the muon identification efficiency is quite low compared to electrons, and therefore the muon+muon final state has a very low yield at $\mathrm{D} \varnothing$.

\subsection{Motivation for a Lepton + Track Selection}

In this analysis, dilepton events were identified by searching for events where one lepton was correctly identified by the detector and the second lepton is identified as an isolated track in the tracking system. The goal of the analysis is to identify dilepton events which would be missed by more traditional analyses that require both leptons to be fully identified. High $p_{T}$ tracks provide a clean signature for a charged lepton so long as tracks contained within jets are identified and removed using isolation requirements. These so-called lepton+track events can then be combined with the fully reconstructed dilepton events as a way of increasing the overall sensitivity to top pair production.

Figure 1 shows the design of the $\mathrm{D} \varnothing$ detector. The detector will be discussed in greater detail below in Section 3, but for now it is important to note a few features. The $\mathrm{D} \varnothing$ calorimeter is designed using a central barrel with two endcaps, 


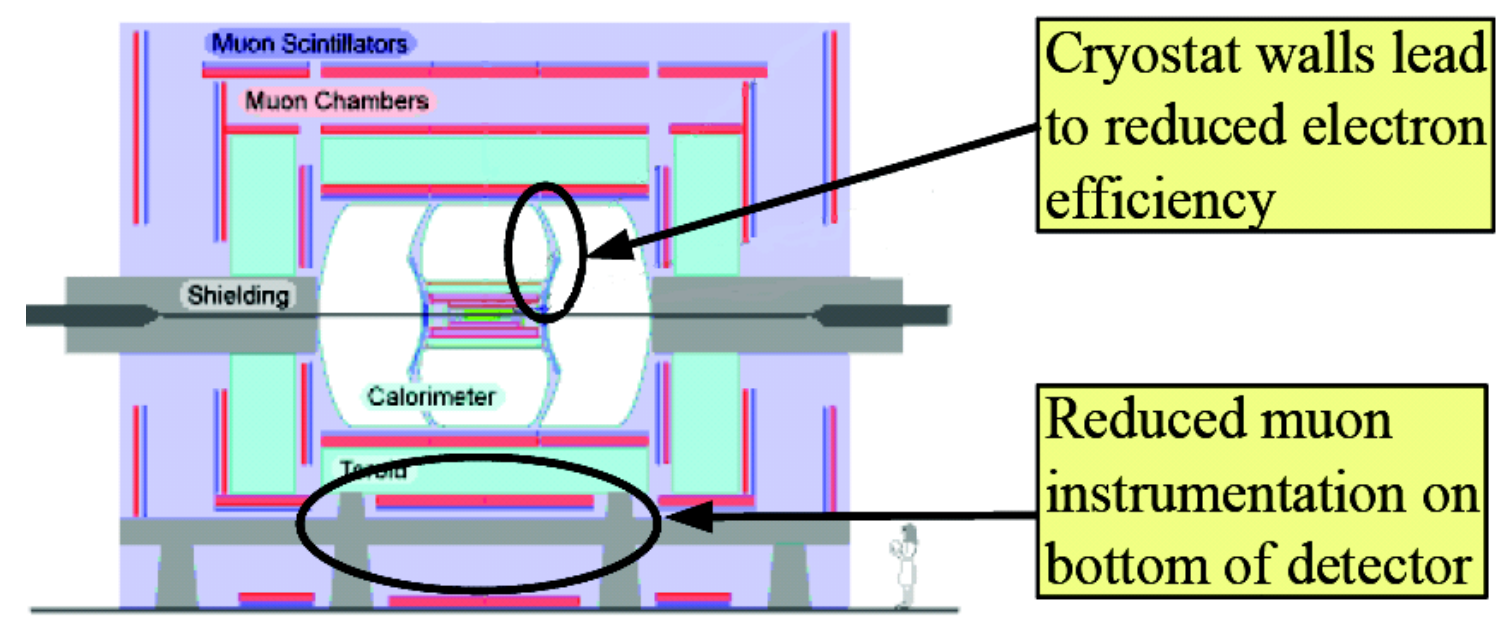

Figure 1: Diagram of the D $\varnothing$ detector, showing the cryostat wall in the calorimeter and the reduced muon coverage on the bottom of the detector.

and the presence of the cryostat walls surrounding each section leads to an angular region which has little instrumentation and therefore poor electron detection efficiency. Furthermore, on the underside of the detector is an area where, due to structural support and space limitations, muon chamber instrumentation is limited and the muon reconstruction efficiency is low. Other effects, such as a particle landing on an intermodular crack, can lead to poorly reconstructed leptons and lost efficiency. However, by loosening the requirements on one lepton to only require an isolated track, it is possible to regain much of this lost efficiency.

Electron bremsstrahlung in the tracking material is another source of decreased electron identification efficiency. Bremsstrahlung can change the shower shape of an electron sufficiently to cause the electron to be misidentified, or the bremsstrahlung photon can land outside the electron's calorimeter cluster entirely, which reduces the efficiency of the calorimeter energy/track momentum matching which is used in the electron identification criteria, as discussed in Section 4.1. The track-based lepton identification used in this analysis does not rely on calorimeter information, and therefore does not suffer from these inefficiencies. As the more 
massive muon is less likely to undergo bremsstrahlung than the electron, this is only a small effect on muon identification efficiency.

Looser lepton identification requirements can also help to reduce the total systematic error on the combined dilepton measurement. Identification cuts must be simulated in the Monte Carlo, and differences between the material description in the simulation and the actual detector leads to systematic errors. Looser identification requirements reduces the sensitivity to detector simulation, and generally leads to lower overall systematic errors.

Figure 2 shows an example of a lepton+track event. The event displayed is a dielectron event, but the marked electron failed one of the acceptance cuts. In this case, it failed a cut on the angle between the electron and the beamline - it is too far forward in the direction of the beam. Acceptance cuts on electrons are discussed fully in Section 4.1. The electron can still be identified, however, by the high momentum track. The remaining tracks in the event are either charged particles contained within the quark jets, or they are low momentum tracks associated with unimportant fragments of the proton-antiproton collision. Sources for high momentum tracks isolated from other tracks include electrons, muons, and taus which decay into a single charged hadron. However, due to calorimeter isolation cuts designed to reject tracks associated with jets, hadronically decaying taus are typically rejected.

A brief outline of this thesis is as follows. Chapter 2 provides an overview of the Standard Model and in particular top quark phenomenology. The DØ detector will be discussed in Chapter 3, and the methods used to identify particles using

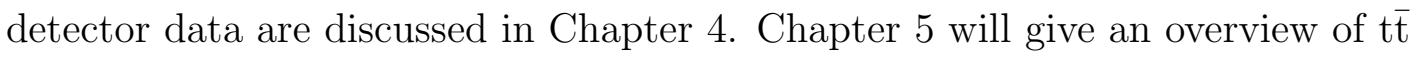
event identification using a lepton+track selection. Chapter 6 covers the data sample used and the background estimation. Chapter 7 goes over the systematic error estimation, and the final results are presented in Chapter 8. 


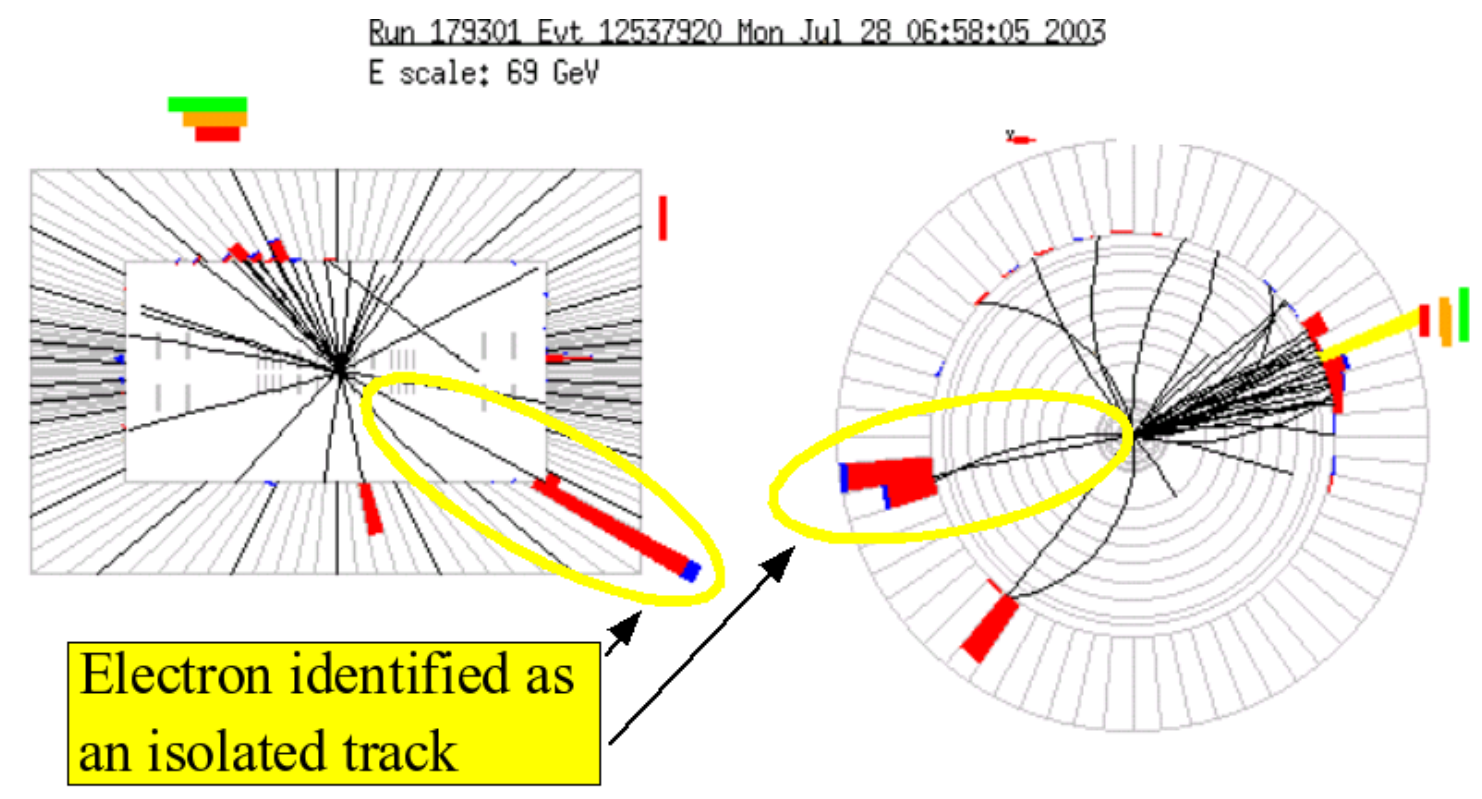

Figure 2: An event display for an example lepton+track event. Thee left-hand side shows an $\mathrm{r}-\mathrm{z}$ view while the right-hand side is an $\mathrm{r}-\phi$ view. Colored bars represent the energy deposited in the calorimeter, where red is electromagnetic energy and blue is hadronic energy, and tracks reconstructed by the central tracker are shown. Colored rectangles outside the calorimeter display represent hits in the muon system. The indicated electron is the electron which was identified by its isolated track. 


\section{Standard Model}

The Standard Model of particle physics provides the best known description of fundamental particles and their interactions, and has thus far never been clearly contradicted by experimental observation. There are twelve fermions in the Standard Model. Six of them are quarks, named up (u), down (d), charm (c), strange (s), top (t), and bottom (b). The up, charm, and top quarks all have an electric charge of $+2 / 3$ while the down, strange, and bottom quarks have charge $-1 / 3$. The remaining six fermions are the leptons: the electron, electron-neutrino, muon, muon-neutrino, tau, and tau-neutrino. The neutrinos are electrically neutral while the other three leptons have charge -1 . The fermions interact via the four known gauge forces: the strong force, mediated by the gluon, the electromagnetic force, mediated by the photon, the weak force, mediated by the charged $\mathrm{W}$ boson and neutral Z boson, and the gravitational force, mediated by the as-yet-unobserved graviton. Quarks and leptons can change their flavor only by emitting or absorbing the charged current $\mathrm{W}$ boson, which allows for the decay of heavier fermion species into lighter fermions.

The top quark was discovered in 1995 by the CDF and D $\varnothing$ collaborations at the Tevatron collider ([2], [3]). Most top quarks at the Tevatron are produced through the strong interaction, which conserves quark flavor, and are therefore produced as top quark-antitop quark pairs. As the valence quarks inside the proton have the highest momentum fraction, the dominant production mechanism for the top quark at the Tevatron is from quark-antiquark collisions, as shown in Figure 3. Some top quarks pairs (about 15\% of the total top pair production at the Tevatron, according to the Standard Model) are also produced through gluon-gluon processes. The expected next-to-next-to-leading order (NNLO) cross section for a top mass of $175 \mathrm{GeV}$ and a center-of-mass energy of $2 \mathrm{TeV}$ is $6.77 \pm 0.42 \mathrm{pb}[5]$. 


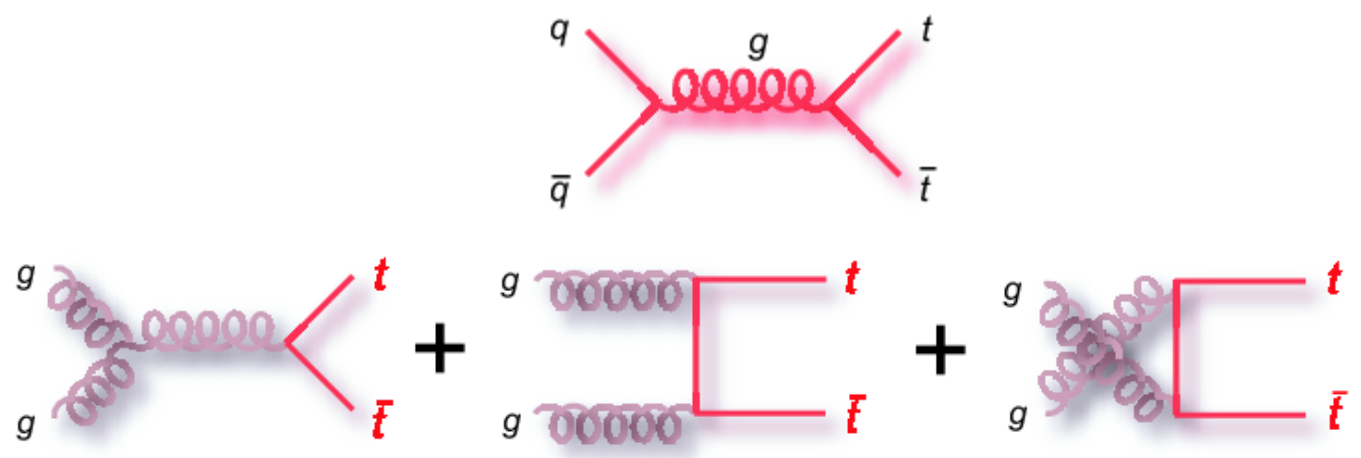

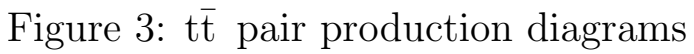

It is also possible to produce single top quarks through weak interaction processes. The cross section for single top production is expected to be $1.98_{-0.18}^{+0.23} \mathrm{pb}$ at the Tevatron for a top mass of $175 \mathrm{GeV}$ based on Standard Model predictions ([6], [7]). Even though the cross section is only slightly less than the top pair production, the fact that there is only one top decay in the event makes it is much more difficult to separate from background. This production mechanism for top quarks will not be of concern in this paper.

For a top quark mass greater than the $\mathrm{W}$ mass, the dominant decay mode for the top quark is into an on-shell $\mathrm{W}$ boson and a bottom quark $(t \rightarrow W s$ and $t \rightarrow W d$ decays are also allowed in the Standard Model, but these are expected to account for only on the order of $0.1 \%$ and $0.01 \%$ of all decays, respectively). The $\mathrm{W}$ boson can decay either into lepton pairs (an electron, muon, or tau together with their antineutrino counterparts) or into quark-antiquark pairs from the first and second generations of quarks (up, down, strange, and charm). Quarks come in three colors (red, green, blue), each of which appears with equal probability during W decay. Since the masses of these quarks and leptons are much less than the $\mathrm{W}$ mass, all weak interaction eigenstates occur at approximately the same rate (weak eigenstates in the quark sector are a rotation of the mass eigenstates $\mathrm{u}, \mathrm{d}, \mathrm{s}$, and $\mathrm{c}$ ). 
Since the top quark's discovery, many measurements have been taken of its properties at the CDF and D $\varnothing$ detectors. Intense efforts have been made to measure top properties for a number of reasons:

- Since the top quark is by far the most massive known fermion, top quark studies probe a higher energy scale than studies of any other fermion.

- Top quark loops and Higgs boson loops in the $\mathrm{W}$ boson propagator shift the mass of the $\mathrm{W}$ boson. Therefore, precision measurements of the top mass and W mass can be used to put constraints on the mass of a Standard Model Higgs boson.

- The top quark has an extremely short decay time, on the order of $10^{-25}$ seconds according to the Standard Model. This timescale is shorter than the typical timescale of strong interactions, which means that unlike the other five quarks the top quark does not have time to form a bound state with other quarks. Because there are not expected to be any bound t $\bar{t}$ states, any observed resonance phenomena would indicate the existence of an unexpected

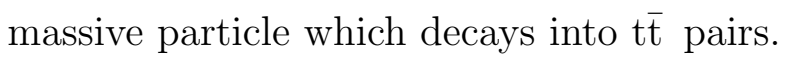

- Another result of the top's rapid decay is that it will typically decay before participating in strong interactions, which allows for the studying of the underlying dynamics of the decay process, such as spin correlations.

- Measurement of the branching fraction $B(t \rightarrow W b)$ allows one to probe the $V_{t b}$ element of the Cabibbo-Kobayashi-Maskawa (CKM) matrix.

- The top quark may have flavor changing neutral current (FCNC) decay channels into charm or up type quarks. FCNCs are highly suppressed loop processes in the Standard Model but are tree level processes in many beyond the Standard Model theories. 
- Since the top quark has the largest mass of all known fermions, it interacts the strongest with the Higgs boson and thus top measurements are sensitive to the Higgs sector. For instance, the existence of a charged Higgs decay channel $t \rightarrow H^{+} b$ would result in a measured cross section below the Standard Model expectations, while other models such as technicolor predict extra production mechanisms which will give a higher than expected cross section [4].

- Top quark pair production is well described by perturbative QCD and it is therefore one of the most precisely predicted QCD processes, making it an excellent testing grounds for Standard Model physics.

- The top's mass and cross section are interrelated. Measurements of the top's cross section can, when combined with Standard Model theory, be used to predict the top quark's mass. These predictions can then be compared with measurements of the top mass from direct measurements using event kinematics as a test of the Standard Model.

Figure 4 displays the predicted cross sections for a selection of physical processes observed at a hadron collider. At the Tevatron energy of $1.96 \mathrm{TeV}$, the cross section for top quark processes $\sigma_{t}$ is around 10 orders of magnitude below the total p $\overline{\mathrm{p}}$ cross section. Furthermore, it is far below the bottom quark production cross section $\sigma_{b}$, as well as the cross sections for weak interactions $\sigma_{W}$ and $\sigma_{Z}$. Heavy flavor bottom quark processes can be misidentified as top events, since top quarks are often identified by their decay into bottom quarks, and weak interaction processes involving heavy $\mathrm{W}$ and $\mathrm{Z}$ bosons can also be very similar to top quark events because the $\mathrm{W}$ and $\mathrm{Z}$ masses are on the same order as the top's mass, making these events kinematically similar. Thus, the top's comparatively small cross section makes rejection of these backgrounds extremely important. 


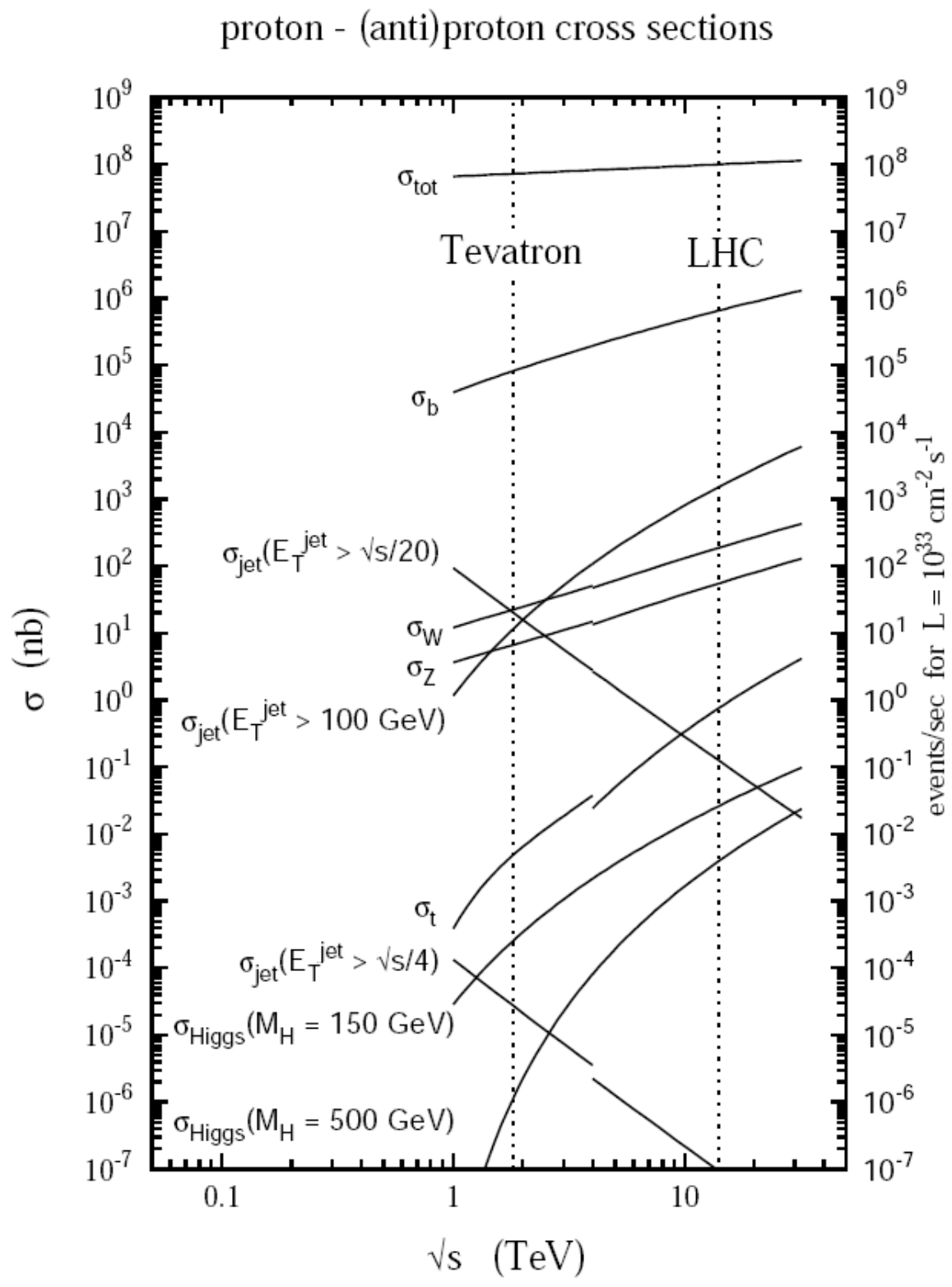

Figure 4: Theoretical predicted cross section versus center-of-mass collision energy for various physical processes. The collision energies for the Tevatron and the LHC are indicated. 


\section{The Tevatron and the DØ Detector}

This section is a brief description of the Tevatron accelerator and the $\mathrm{D} \varnothing$ detector. Further information can be found in $([8]-[13])$.

\subsection{Fermilab and the Tevatron Accelerator}

Due to its large mass, top quark production requires a high center-of-mass energy. Currently, the only accelerator capable of producing such high energy collisions is the Tevatron accelerator at Fermi National Labs. Fermilab is located in Batavia, Illinois, about 40 miles west of Chicago.

The Tevatron is a proton-antiproton collider ([8] - [13]). Protons are used because it is easier to accelerate the relatively massive proton to high energy and sustain that energy in a circular orbit, as a massive particle such as the proton will lose significantly less energy to synchrotron radiation than a low mass particle such as the electron. The tradeoff, though, is that the proton is not a fundamental particle but is composed of constituent quarks and gluons, complicating data analysis. In any given hard-scattering interaction, the colliding particles will only have a fraction of the total $\mathrm{p} \overline{\mathrm{p}}$ center-of-mass energy.

The data set used for this measurement is the "Run 2a" data set taken by the $\mathrm{D} \varnothing$ detector. This data was taken at a center-of-mass energy of $1.96 \mathrm{TeV}$ from August 2002 to March 2006.

A diagram of the layout of the Fermilab accelerator facility is shown in

Figure 5. The first stage of acceleration is the magnetron [14] which is used to create a beam of $\mathrm{H}^{-}$ions. The inner wall of the magnetron is a cathode while the outer wall is an anode, and it is placed within a uniform magnetic field. Hydrogen gas is injected into one end of the chamber, and an extractor plate on the opposite end collects and accelerates the $\mathrm{H}^{-}$ions to the next stage. The Cockroft-Walton 


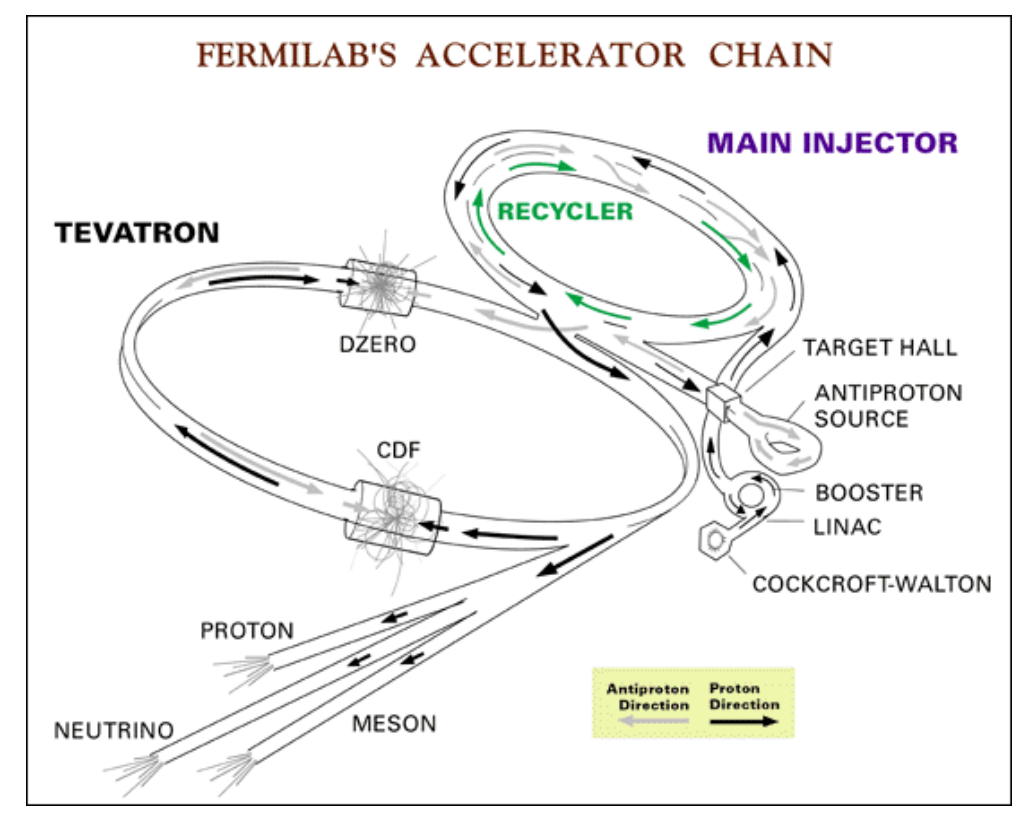

Figure 5: Diagram of the Tevatron accelerator

accelerator accelerates the $\mathrm{H}^{-}$ions to an energy of $750 \mathrm{keV}$, and injects them into the Linac [15]. The Linac is a linear accelerator $150 \mathrm{~m}$ long, which uses five radio frequency $(\mathrm{RF})$ cavities to accelerate the $H^{-}$ions to an energy of $400 \mathrm{MeV}$. From the Linac the ions are passed through a carbon foil, which strips off the electrons to create a proton beam.

The next stage of acceleration is the Booster [16], which is a synchrotron accelerator of radius $75 \mathrm{~m}$. Synchrotron accelerators use RF cavities to accelerate charged particles, and bending magnets spaced around the ring are ramped up as the particles accelerate to maintain the same radius of the particles' orbits. The Fermilab Booster contains 96 dipole and quadrapole magnets, as well as 17 RF cavities. The final acceleration energy of the Booster is $8 \mathrm{GeV}$, and protons are passed from the Booster into the Main Injector [17].

The Main Injector is also a synchrotron with a radius of about $1 \mathrm{~km}$ and it accelerates proton beams to an energy of $120 \mathrm{GeV}$ to $150 \mathrm{GeV}$. From here, $150 \mathrm{GeV}$ 
protons can be injected into the Tevatron itself for final collisions or $120 \mathrm{GeV}$ proton beams can be sent to the antiproton source.

Antiprotons are produced and stored while the Tevatron is colliding beams. In the antiproton source a $120 \mathrm{GeV}$ proton beam from the Main Injector collides with a nickel target. The subsequent nuclear interactions produce a large number of hadrons, some of which will be antiprotons. The shower of particles produced by the collision is passed through a cylindrical lithium "lens" with a $0.5 \mathrm{MA}$ current. The azimuthal magnetic field produced by the current serves to focus negatively charged particles. After the lens, a bending magnet is used to select negatively charged particles with an energy of around $8 \mathrm{GeV}$. From there the antiprotons are passed into the Debuncher, which converts the incoming "bunched" beam into a continuous beam with a smaller momentum width. The antiproton beam is then cooled through stochastic cooling, where a series of small kicks from an electric field on each revolution is used to eventually force the antiprotons into the desired orbit. From the Debuncher the antiprotons are sent into the Accumulator for storage and further stochastic cooling.

Once a sufficient number of antiprotons have been gathered in the Accumulator, they are sent to the Recycler for further cooling and storage. The Recycler is a fixed-energy storage ring located directly below the Main Injector. Antiproton beams in the Recycler are first cooled through stochastic cooling, and then further cooled through electron cooling. In electron cooling, a cold (low emittance) electron beam circulates with the antiproton beam at the same velocity. Through standard thermodynamic processes, heat is transfered from the hotter antiproton beam to the colder electron beam. The electron beam operates at an energy of chosen to be velocity-matched to the antiproton beam. Electron cooling was first achieved in the Fermilab Recycler on July 15, 2005 [18], and it was the first demonstration of electron cooling at these energies. It has been a part of standard 
Tevatron operations since August 2005, and it has resulted in a substantial increase in luminosity.

Before a run begins, antiproton beams are transferred from the Recycler to the Main Injector. The Main Injector accelerates the antiprotons to a final energy of $150 \mathrm{GeV}$ shortly before injection into the Tevatron.

The Tevatron itself is a synchrotron utilizing superconducting electromagnets - 775 dipole magnets and 216 quadrapole magnets in all. Proton and antiproton beams circulate in opposite directions in the Tevatron; a typical run uses around $10^{11}$ protons and $10^{10}$ antiprotons. The final collision energy is $0.98 \mathrm{TeV}$ per beam, or a total center-of-mass energy of $1.96 \mathrm{TeV}$. The beam is divided into three superbunches, each of which is composed of 12 bunches. The beams cross at two locations on the ring, designated $\mathrm{B} \varnothing$ (the location of the CDF detector), and DØ. Bunch crossings occur every 396 ns, while the superbunches themselves are separated by about $2.6 \mu \mathrm{s}$. Some of the key parameters for the Tevatron are summarized in Table 1.

\begin{tabular}{|l|r|}
\hline Center-of-Mass Energy & $1.96 \mathrm{TeV}$ \\
Radius & $1 \mathrm{~km}$ \\
Peak Luminosity & $\sim 300 \times 10^{30} \frac{1}{\mathrm{~cm}^{2} \mathrm{~s}}$ \\
Number of Bunches & $36 \mathrm{p}, 36 \mathrm{pbar}$ \\
Bunch Length & $50 \mathrm{~cm}$ \\
Transverse Beam Radius & $40 \mu \mathrm{m}$ \\
Anti-proton Stacking Rate & $6-10 \frac{\mathrm{mA}}{\mathrm{h}}$ \\
RF Frequency & $53 \mathrm{MHz}$ \\
Period between Beam Crossings & $396 \mathrm{~ns}$ \\
\hline
\end{tabular}

Table 1: Parameters of the Tevatron.

\subsection{DØ Detector}

The $\mathrm{D} \varnothing$ detector is a multipurpose detector in operation since 1992 [11]. From 1996 to 2001 the Tevatron as well as the D0 detector underwent a substantial upgrade 


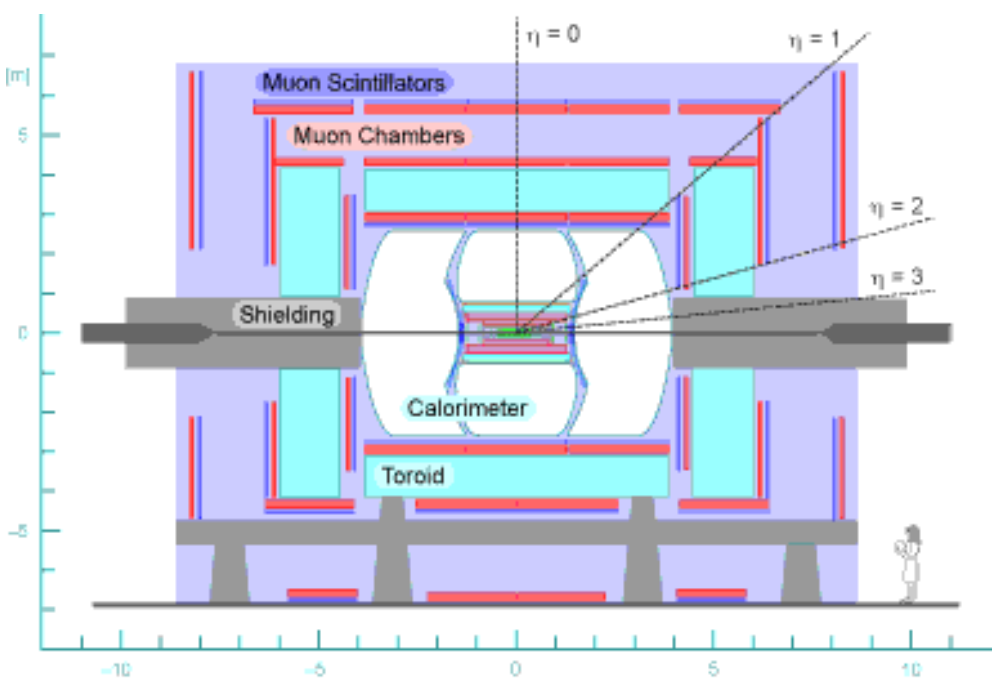

Figure 6: Diagram of the $\mathrm{D} \varnothing$ detector.

[19]. A diagram of the detector is shown in Figure 6. DØ was designed for the study of high mass final state particles, in particular the $\mathrm{W}$ and $\mathrm{Z}$ bosons as well as searches for the top quark and Higgs boson. It has nearly $4 \pi$ solid angle coverage, with uninstrumented regions in the forward and backwards directions due to the beampipe. The detector is composed of multiple subdetectors. The tracking system is the innermost subdetector closest to the collision region and is used to reconstruct the track of electrically charged particles passing through. A solenoid magnet also allows for a momentum measurement based on the curvature of the track. Outside the tracking system is the calorimeter, which measures the energy deposited from electromagnetic and hadronic showers. The outermost section of the detector is the muon system.

\subsubsection{Coordinates}

Before discussing the detector's design, it is useful to first define the coordinate system that we will be using. The z-axis is taken to be in the direction of the proton beam. The positive $\mathrm{y}$ direction is upward, and the $\mathrm{x}$ direction is determined by the 
right hand rule. The cylindrical radius $r$ is defined as $r=\sqrt{x^{2}+y^{2}}$, the azimuthal angle $\phi$ is defined relative to the $\mathrm{x}$ axis, and the pseudorapidity $\eta$ is defined as

$$
\eta=-\ln \left(\tan \left(\frac{\theta}{2}\right)\right)
$$

where $\theta$ is the polar angle defined relative to the z-axis. In the ultra relativistic limit $E>>m$, the pseudorapidity approaches the true rapidity, which is

$$
y=\frac{1}{2} \ln \left(\frac{E+p_{z}}{E-p_{z}}\right)
$$

Pseudorapidity is a convenient variable for use in experimental particle physics because intervals of true rapidity are Lorentz invariant, and also because particle multiplicity tends to be roughly constant in $\eta$. The latter fact suggests a detector design that is segmented into equal $\eta$ intervals rather than $\theta$ intervals.

\subsection{Tracking}

The DØ tracking system has three major components: the Silicon Microstrip Tracker (SMT), the Central Fiber Tracker (CFT), and a solenoid magnet with a field strength of $2 \mathrm{~T}$. The tracking system measures the momentum of electrically charged particles created in the $\mathrm{p} \overline{\mathrm{p}}$ collision. Particle energy is determined by measuring the radius of curvature of the particle's trajectory in the solenoid field. The tracking system is build using a low $\mathrm{Z}$ material to minimize the deflection of a particle passing through this subsystem.

The innermost section of the tracking system closest to the collision region is the Silicon Microstrip Tracker ([20] - [22]). The SMT provides tracking data out to around $|\eta|=3$. In an ideal tracking detector, the tracks would be roughly perpendicular to the detector planes, however this is not possible at D $\varnothing$ as the collision region is relatively long $(\sigma \approx 30 \mathrm{~cm})$. Therefore, the SMT has been 


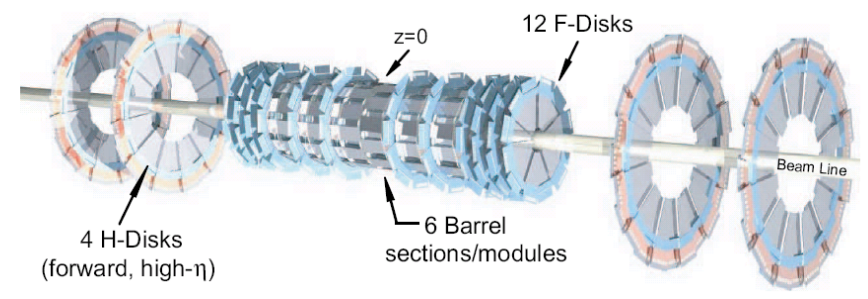

Figure 7: Diagram of the DØ Silicon Microstrip Tracker

designed using a set of barrels and disks to accomodate the long interaction region as shown in Figure 7. The disks will primarily detect particle tracks at high $\eta$, while the barrel is more useful for the lower $\eta$ tracks as these are nearly perpendicular to the beamline.

The SMT barrel is four layers thick, and is divided into six modules. Two of the layers are single-sided detectors and the remaining two are double sided. The active region on each of the silicon detectors is segmented into a set of parallel strips. On the double-sided detectors one side of the strip is an axial layer oriented along the beamline, while the other side is at a stereo angle of either 2 or 90 degrees. The silicon used in the SMT barrel is $300 \mu \mathrm{m}$ thick n-type wafers. $p^{+}$ implants, which are used to collect the charge, are separated from an aluminum coating by a resistive layer. The image charge on the aluminum is then read out.

On the high $|z|$ end of each module is a disk, called an F disk. F disks are composed of 12 double-sided wedge-shaped silicon detectors. The entire construction is capped by three $\mathrm{F}$ disks as well as two $\mathrm{H}$ disks on the north and south ends of the detector. $\mathrm{H}$ disks contain 24 wedges, and each wedge is made from two single-sided detectors. The $\mathrm{H}$ disks are positioned 110 and $121 \mathrm{~cm}$ from the center of the detector.

The Central Fiber Tracker [23] occupies the region from 20 to $52 \mathrm{~cm}$ from the beamline and provides tracking coverage out to $\eta \sim 2$. The CFT operates by using scintillating fibers each of which has a diameter of $835 \mu \mathrm{m}$. Each fiber is 
composed primarily of polystyrene doped with paraterphenyl ( $1 \%$ by weight), which is an organic florescent dye. Excitation from an electrically charged particle sets up a chain of dipole-dipole interactions which pass energy through the polystyrene to the paraterphenyl, which then floresces at a wavelength around $340 \mathrm{~nm}$ after a few ns. $340 \mathrm{~nm}$ radiation can only penetrate a few hundred microns through polystyrene, and thus the fibers are also doped (at $1500 \mathrm{ppm}$ ) with 3-hydroxyflavone. 3-hydroxyflavone shifts the $340 \mathrm{~nm}$ radiation to a wavelength of $530 \mathrm{~nm}$, which is transmitted easily through the fiber. The signal from each fiber is carried through a pure polystyrene fiber (not doped with paraterphenyl or 3-hydroxyflavone) to a solid state photodetector capable of detecting single photons.

The CFT detector is arranged as eight concentric cylinders. The two innermost cylinders are $1.66 \mathrm{~m}$ in length, while the outermost six are $2.52 \mathrm{~m}$ long. Each cylinder supports two double layers of scintillating fibers known as the axial layers and the stereo layers. Within each double layer of fibers, the outer layer is offset from the inner layer by half of a fiber diameter, thereby filling in gaps in the coverage of the inner layer. For the axial layers, each fiber is laid out parallel to the beam direction. The stereo layers, on the other hand, wind around the beamline at an azimuthal angle of $\pm 3^{\circ}$. For each of the eight cylinders, the innermost layers of fibers are the axial layers and the outer layers are the stereo layers. The innermost cylinder has its stereo layers oriented at $+3^{\circ}$, and the stereo layers alternate $-3^{\circ}$, $+3^{\circ},-3^{\circ}$, etc., after that.

The solenoid [24] is a superconducting magnet operating at a temperature of 4.7 K and lies outside of the SMT and CFT. The magnetic field allows for measurements of particle momentum within the tracking system. The solenoid has a $1.42 \mathrm{~m}$ diameter with a length of $2.73 \mathrm{~m}$. The field is created with a $4825 \mathrm{~A}$ current, and contains a total energy of 5.6 MJ. The solenoid is approximately one radiation length thick for a particle passing through it at low $\eta$. 
The resolution of the $\mathrm{D} \varnothing$ tracker is roughly a Gaussian in $1 / p_{T}$ with a width given by:

$$
\frac{\sigma_{1 / p_{T}}}{1 / p_{T}}=\sqrt{A^{2} \frac{p_{T}^{2}}{L^{4}}+\frac{B^{2}}{L \sin \theta}}
$$

The parameter $A$ corresponds to the effect of the hit resolution on the track's $p_{T}$. High $p_{T}$ tracks have a larger radius, which results in a greater relative error from hit position resolution effects, and thus the $A$ term determines the high $p_{T}$ behavior of the tracking resolution. The $B$ term is the effect of multiple scatterings on the track resolution, and this term dominates the low $p_{T}$ regime. $L$ is an approximation to the distance perpendicular to the solenoid's magnetic field where the track leaves the tracker:

$$
\begin{aligned}
L=1, & \text { if }\left|\eta_{\text {phys }}\right|<1.62 \\
L=\frac{\tan \theta_{\text {phys }}}{\tan \theta_{C F T}}, & \text { otherwise }
\end{aligned}
$$

Along with the Gaussian resolution described above, a further linear scaling is needed to describe the tracking resolution:

$$
p_{T}(\text { smear })=C p_{T}^{\prime}
$$

where $p_{T}$ is the final, measured transverse momentum and $p_{T}^{\prime}$ only includes the effects of the Gaussian resolution described above. The parameter $C$ accounts for two effects - energy lost as the particle travels through detector material, and imperfections in the simulation of the solenoid field.

The parameters $A, B$, and $C$ were determined by smearing the particles' momenta in Monte Carlo events in order to match the momentum distributions 
observed in data [26]. Two datasets were used for this process. The first was a sample of $\mathrm{Z} \rightarrow \mu \mu$ events. Muon tracks in $\mathrm{Z} \rightarrow \mu \mu$ decays will generally have high momentum, where the effects of the tracking resolution parameter $A$ dominates. The parameter $C$ was also determined from these events. These parameters were evaluated by matching the resonance peak in Monte Carlo to that observed in data. The Monte Carlo sample was first weighted to normalize it to the data, and then the $A$ and $C$ parameters were adjusted to minimize the $\chi^{2}$ :

$$
\chi^{2}=\sum_{i} \frac{\left(N_{i}^{\text {data }}-N_{i}^{M C}\right)^{2}}{\left(\sigma_{i}^{\text {data }}\right)^{2}+\left(\sigma_{i}^{M C}\right)^{2}}
$$

where the summation is taken over the mass bins $i$. A similar procedure was then performed for a sample of events at the $\mathrm{J} / \psi$ (a meson with quark composition $c \bar{c})$ resonance. The muons in these events have low momentum, which allows for a measurement of the parameter $B$ as well as a cross-check of $C$. The final results are:

$$
\begin{aligned}
& A=0.0029 \pm 0.0010 \mathrm{GeV}^{-1} \\
& B=0.0254 \pm 0.0008 \\
& C=0.992 \pm 0.003
\end{aligned}
$$

\subsection{Preshower}

Outside of the solenoid lies the preshower detector. The preshower detector is primarily used in electron identification. The Central Preshower detector (CPS) covers the region out to $|\eta|<1.3$ and the Forward Preshower covers the $\eta$ range $1.5<|\eta|<2.5$. The preshower detectors use triangular scintillators composed of a polystyrene/paraterphenyl material. 
The CPS detector has three concentric layers of scintillators. One of the CPS layers is an axial layer while the remaining two are stereo layers with stereo angle $\pm 20^{\circ}$. Before the CPS lies the solenoid, which is about 0.9 radiation lengths thick, followed by a lead radiator which adds another 1 radiation length, for a total of about two radiation lengths. At higher $|\eta|$ this increases to around four radiation lengths. Electromagnetic objects (electrons, photons), will shower in the material preceeding the CPS, and this showering is then detected by the preshower detector. Hadrons will not form showers because the material preceeding the CPS is short compared to the hadronic interaction length.

The FPS consists of a double layer of scintillators known as the Minimum Ionizing Particle (MIP) layers, followed by a steel absorber two radiation lengths thick, outside of which lies two more layers of scintillators called the shower layers. All FPS scintillator layers are stereo layers oriented at $\pm 22^{\circ}$. Both electrons and photons will form an electromagnetic shower within the steel absorber. These showers are generally three scintillator strips wide in the shower layers. Similar to the CPS detector, electrons and photons will shower within the absorber while hadronic particles will not form showers. The MIP layers are used to identify electrons from photons: electrons will have one hit within the MIP layers, while the neutral photon will have no signature.

\subsubsection{Calorimeter}

The DØ calorimeter [27] measures the energy of electrons, photons, and jets. Also, calorimeter data is used to determine the transverse momentum $p_{T}$ of the observed objects, and from this the amount of "missing" transverse energy is calculated which is associated with the unobserved neutrinos in the event.

The $\mathrm{D} \varnothing$ calorimeter is an example of a sampling calorimeter. In a sampling calorimeter, layers of a dense absorber material are alternated with an active 


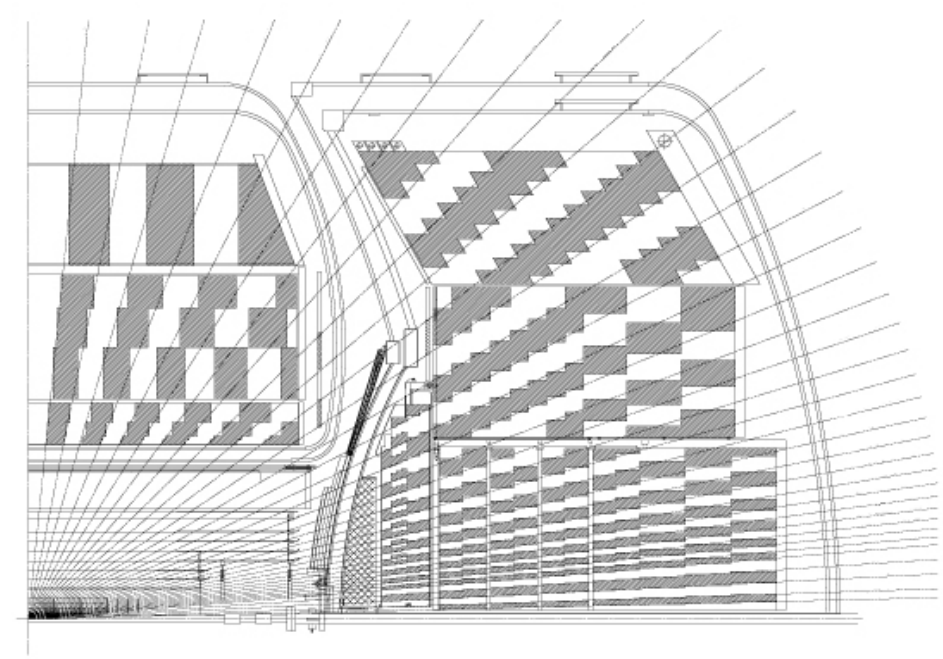

Figure 8: Layout of the readout cells in the $\mathrm{D} \varnothing$ calorimeter.

medium. Most of the energy from energetic particles is deposited in the absorber material, with typically only $1 \%$ to $10 \%$ of energy being deposited in the active medium. For the $\mathrm{D} \varnothing$ detector, the active medium is liquid argon while the absorber is one of uranium, copper, or steel.

Calorimeter cells are arranged in "pseudoprojective towers", as shown in Figure 8. The towers are referred to as being pseudoprojective because, although the centers of the individual cells lie along a ray of constant $\eta$, the boundaries of the cells do not. The size of the towers is $0.1 \times 0.1$ in $\eta$ - $\phi$ space, except in the far forward region where rays of equal $\Delta \eta$ become very close together, so towers in this region must span more than 0.1 in $\eta$ for engineering reasons. Also, the third layer of the electromagnetic calorimeter has a fine resolution of $0.05 \times 0.05$ in $\eta-\phi$, which is used to determine the centroid of an electromagnetic shower to high precision.

As shown in Figure 9, the $\mathrm{D} \varnothing$ calorimeter is divided into three sections: the Central Calorimeter (CC), which covers up to an $\eta$ of around 1, and two End Calorimeters (EC), which extend the $\eta$ coverage to about 4 . Each section of the 


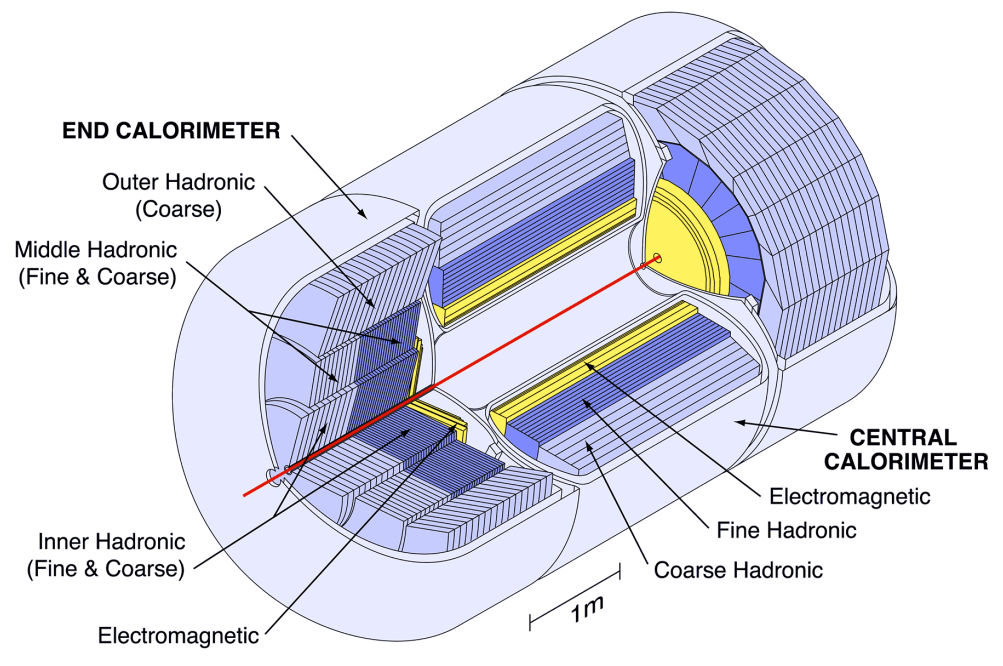

Figure 9: Cutaway diagram of the $\mathrm{D} \varnothing$ calorimeter.

calorimeter is contained within its own cryostat, which keeps the detector operating at a temperature of $80 \mathrm{~K}$. The Central Calorimeter is composed of three concentric shells, with 32 modules in the electromagnetic portion, 16 fine hadronic modules, and 16 coarse hadronic modules. The three shells are rotated with respect to each other such that no ray from the origin falls on more than one intermodular crack. An Inter-Cryostat Detector (ICD), made from a scintillating material, is included between the Central Calorimeter and the two End Calorimeters in order to improve the detector sensitivity in the $0.8<|\eta|<1.4$ range.

A typical unit cell is shown in Figure 10. The signal board is separated from the absorber plate by a $2.3 \mathrm{~mm}$ liquid argon gap on either side and is coated with a fine resistive epoxy. The signal board is held at $2-2.5 \mathrm{kV}$ while the absorber plates are grounded. The drift time for electrons across the argon gaps is around $450 \mathrm{~ns}$.

The innermost four readout layers of the calorimeter form the electromagnetic calorimeter, as electromagnetic objects (electrons and photons) will deposit nearly all of their energy in these layers. High energy electrons in the calorimeter lose most of their energy through bremsstralung, while high energy photons will split into electron-positron pairs. Through bremsstralung and pair 


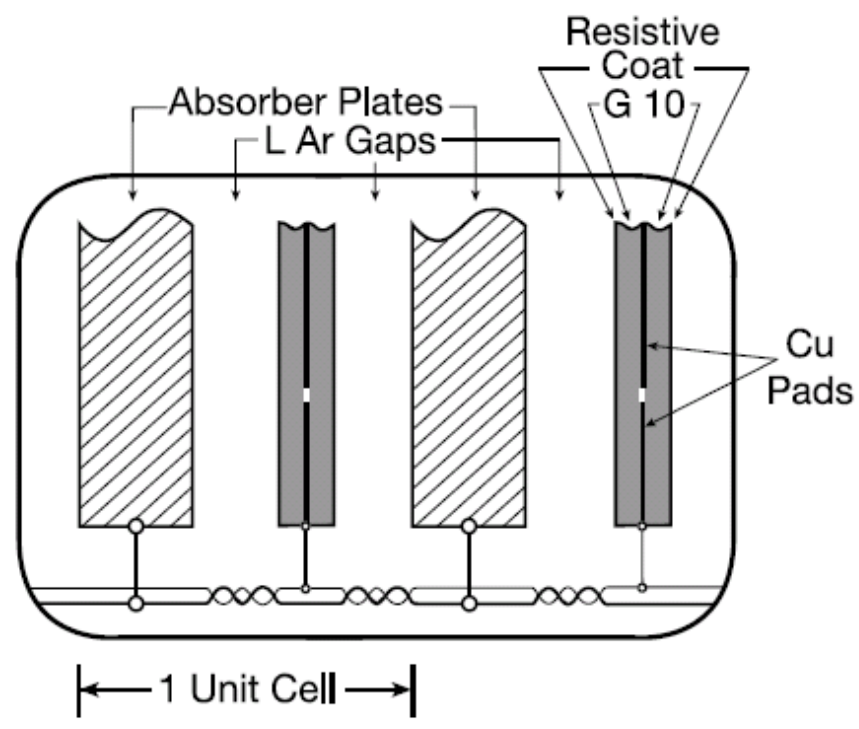

Figure 10: Diagram of a unit cell in the DØ calorimeter.

creation, the number of particles in an electromagnetic shower will grow geometrically. Below the critical energy $E_{c}=800 \mathrm{MeV} /(\mathrm{Z}+1.2)$, electrons will lose more energy through ionization than from bremsstralung [25]. For an electromagnetic shower, the calorimeter material is characterized by the radiation length $X_{0}$, representing the distance an electromagnetic particle must travel in order for it to lose one power of $e$ due to ionization. The radiation length is approximately $[25]$

$$
X_{0} \sim \frac{716.4 A}{Z(Z+1) \ln (287 / \sqrt{Z})} \mathrm{g} / \mathrm{cm}^{2}
$$

Dense materials are generally used in calorimetry in order to decrease the size required to capture all of a shower's energy. Uranium, which has a radiation length of $0.32 \mathrm{~cm}$, was chosen for the DØ electromagnetic calorimeter. In the Central Calorimeter, the four electromagnetic layers are 2.0, 2.0, 6.8, and 9.8 radiation lengths deep, while the End Calorimeter electromagnetic layers are 0.3, 2.6, 7.9, and 9.3 radiation lengths. For the End Calorimeters, the wall of the 
cryostat plus the innermost electromagnetic absorber layer has a total thickness of approximately 2 radiation lengths.

Outside of the electromagnetic calorimeter is the hadronic calorimeter, which is used for reconstruction of hadronic jets. The fine hadronic calorimeter consists of the innermost layers of the hadronic calorimeter, and outside of this is the coarse hadronic calorimeter. The coarse hadronic layers have greater measurement uncertainty than the fine hadronic cells and are needed to provide sensitivity to hadronic showers that penetrate the fine hadronic calorimeter.

Hadronic showers penetrate much deeper into the calorimeter than an electromagnetic shower and are also much wider laterally. Hadrons passing through the calorimeter will transfer most of their energy to the atomic nuclei, and the nuclear interactions can produce secondary hadrons if sufficient energy is available. These secondary hadrons will include some neutral pions, which quickly decay into two photons. This gives an electromagnetic component to hadronic showers. The key characteristic of a material with regards to hadronic showers is the nuclear interaction mean free path $\lambda_{I}$, which is the distance a hadronic particle must travel through the medium to have a probability of $(e-1) / e$ of having an inelastic collision with a nucleus in the material. An approximation of the nuclear mean free path is given by [25]:

$$
\lambda_{I} \sim 35 A^{\frac{1}{3}} \mathrm{~g} / \mathrm{cm}^{2}
$$

Typically, $\lambda_{I}>>X_{0}$. Most of the hadronic calorimeter is made with depleted uranium, which has a nuclear mean free path of $10.5 \mathrm{~cm}$. In the Central Calorimeter, the three fine hadronic layers (see Figure 9) have thicknesses of 1.3, 1.0, and 0.76 interaction lengths, with an additional 3.2 interaction length coarse hadronic layer, which uses copper as the absorber material. The End Calorimeter is further subdivided into the Inner Hadronic (highest $\eta$ ), Middle Hadronic, and Outer 
Hadronic (lowest $\eta$ ) sections. The Inner Hadronic has 4 layers, each of which has 1.76 interaction lengths of uranium, and a 4.1 interaction length coarse layer made from copper. The Middle Hadronic section similarly has 4 uranium layers (0.9 interaction lengths each) outside of which is a coarse copper layer 4.1 lengths deep. Finally, the Outer Hadronic section is made using stainless steel as the absorber, and each layer can be up to 6 interaction lengths.

The calorimeter's response has been tested using test beams with energies ranging from 10 to $150 \mathrm{GeV}$ using both electron and pion beams ([28] - [30]). The resolutions have been determined to be

$$
\begin{aligned}
\frac{\sigma(E)}{E} & =\frac{16 \%}{\sqrt{E(G e V)}} \oplus 0.3 \% \text { (electrons) } \\
\frac{\sigma(E)}{E} & =\frac{41 \%}{\sqrt{E(G e V)}} \oplus 3.2 \% \text { (pions) }
\end{aligned}
$$

where $\sigma(E)$ is the resolution as a function of the energy $E$, and $\oplus$ means added in quadrature.

\subsubsection{Muon System}

Muons, being substantially more massive than electrons, lose relatively little energy due to bremsstralung in the calorimeter at energies less than a few hundred $\mathrm{GeV}$ [25], and they also do not have strong interactions with atomic nuclei as hadrons do. Muons are also long-lived particles (relatively speaking) and thus they are able to pass through the calorimeter and only lose a small fraction of their energy along the way. No other (known) particle has a substantial probability of exiting the calorimeter (other than the virtually undetectable neutrino), and thus any particle detected beyond the calorimeter can safely be taken to be a muon. 
A schematic of the $\mathrm{D} \varnothing$ muon system is shown in Figure 11. The muon system uses a toroidal magnet with a field strength of 1.9 Tesla to deflect the muons, and the angle of deflection is used to determine the momentum. The toroid is a square annulus $109 \mathrm{~cm}$ thick, located $318 \mathrm{~cm}$ out from the beamline. The Wide Angle MUon System (WAMUS) covers the $\eta$ range out to $|\eta|<1$ while the Forward Angle MUon System (FAMUS) provides coverage from an $\eta$ of 1 out to $\eta=2$. Measurements made with DØ's muon system have much lower precision than those made with the inner tracking system, so the muon system data is generally matched to the tracking data and the high-resolution tracking measurements are used to evaluate the muon's momentum and energy.

The WAMUS has three layers of Proportional Drift Tubes (PDTs), dubbed the A, B, and C layers. Drift tubes in the WAMUS have a $5.5 \times 10$ centimeter cross section and are 2.4 meters long. Each individual cell has a central wire held at high voltage, and as ionization electrons accelerate towards it they cause further ionization which leads to an 'avalanche' of charge that can be detected. The central muon system PDTs use a gas mixture of $84 \%$ argon, $8 \%$ methane, and $8 \% \mathrm{CF}_{4}$, and the drift time is $500 \mathrm{~ns}$. The A layer has four decks of drift cells, except for the bottom portion of the detector where there are only three decks, and is located immediately inside the toroid. The B layer is three decks thick and is immediately outside the toroid, and finally the $\mathrm{C}$ layer is also three decks and is positioned about $1.4 \mathrm{~m}$ away from the toroid. The muon is tracked before and after the toroid and from this the deflection the muon experiences in the toroid is determined and thus its momentum can be calculated. The A layer has a directional resolution of 0.6 mrad and the $\mathrm{B}$ and $\mathrm{C}$ have resolutions of $0.17 \mathrm{mrad}$ and $0.2 \mathrm{mrad}$, respectively. The WAMUS also utilizes layers of scintillators. The cosmic cap and cosmic bottom scintillators lie outside of the outermost PDTs, with the cosmic cap covering the top and sides of the detector and the cosmic bottom covering (unsurprisingly) 


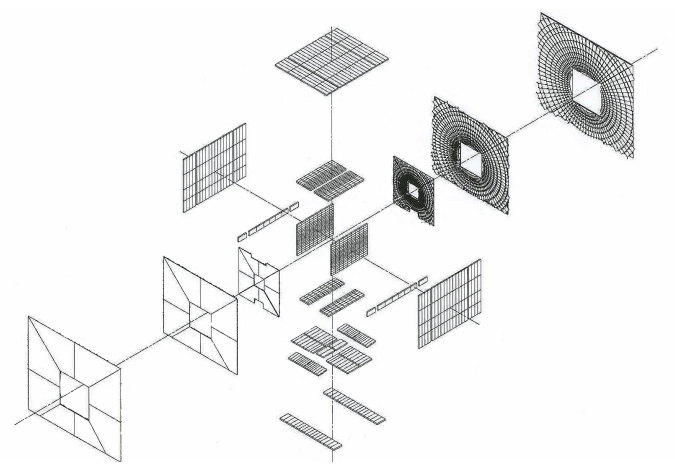

(a)

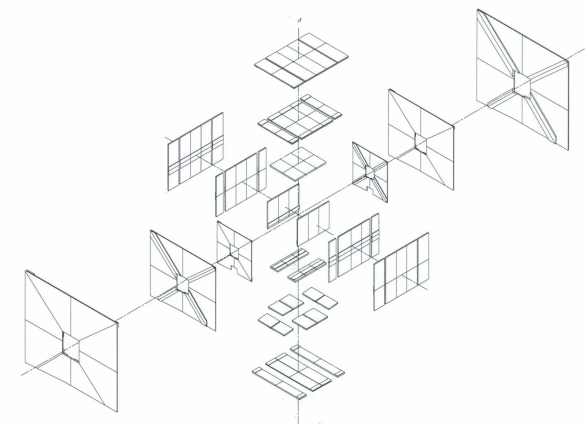

(b)

Figure 11: Diagram of (a) the muon system scintillators and (b) the muon drift chambers.

the bottom portion of the detector. The primary difference between the cosmic cap and cosmic bottom is that the cosmic bottom scintillator strips are oriented with their longest dimension in the z-direction, parallel to the beamline, while the cosmic cap scintillators are oriented azimuthally. The cosmic cap and cosmic bottom allow for very precise timing measurements, which helps reject cosmic ray backgrounds by vetoing events which do not coincide with a beam crossing. An additional layer of scintillators known as the A $\phi$ layer covers the A layer of PDTs and is used both for triggering and background rejection.

The FAMUS extends the $\eta$ coverage to around 2. Due to very high occupancy in the forward region, Mini Drift Tubes (MDTs) are used in this region. The cross section of an MDT is $1 \times 1$ centimeter, and the length of the tubes varies throughout the detector. Three layers of MDTs (A, B, and C) are used, with four planes of tubes in the A layer and three planes each in the B and C layers. A gas mixture of $90 \% \mathrm{CF}_{4}$ and $10 \%$ methane is used, and the drift time for the MDTs is 132 ns. Three layers of scintillators are used for the triggering system. Each layer is divided into octants with 96 detectors each. 
Uncertainties arise both from scattering of the muons inside the toroid material as well as from the hit position resolution of the PDTs. The muon system resolution was determined by comparing dimuon events in data with $\mathrm{Z} \rightarrow \mu \mu$ Monte Carlo events, and then smearing the muon resolution in the Monte Carlo until the width of the $\mathrm{Z}$ mass distribution matched what was observed in data. The momentum resolution is approximately Gaussian in $1 / p$ with [31]

$$
\sigma\left(\frac{1}{p}\right)=\frac{0.18(p-2)}{p^{2}} \oplus 0.003
$$

where $p$ is measured in $\mathrm{GeV}$.

\subsection{Trigger System}

As with all hadron colliders, most of the events observed in the $\mathrm{D} \varnothing$ detector are QCD multijet events involving light quark flavors. Processes with much lower cross sections, such as weak interactions and production of superheavy particles like the top quark, must be identified from the QCD background. Since it is unrealistic to record the data from every single beam crossing due to the high data rate, a triggering system is used to identify events to be recorded [32]. The trigger system performs a rough reconstruction of each event and makes a trigger decision based on the trigger objects (such as calorimeter clusters, tracks, muons, etc.). Each trigger is assigned a prescale, such that frequent events such as QCD multijet processes will be assigned a large prescale, meaning that only one out of a large number of events will be recorded to tape. Less frequent events such as weak interactions will be given a small prescale, such that all or nearly all of these events will be recorded.

Three levels of triggers are used at $\mathrm{D} \varnothing$. The beam crossing rate is $1.5 \mathrm{MHz}$, so the first trigger level, Level 1, must make trigger decisions on this timescale. The Level 1 trigger must therefore use simplified algorithms for the reconstruction of 
objects. Subsequent trigger levels - Level 2 and Level 3 - have more time to make trigger decisions and can therefore perform more complex event reconstruction. The Level 1 trigger system is hardware and firmware-based in order to process events at a sufficiently high rate, while the Level 2 trigger uses both hardware and preprocessors. The Level 3 trigger system is software-based and is performed on a CPU farm.

The Level 1 trigger decision is made using data from the CFT axial layers, preshower detectors, calorimeter towers, and the drift tubes and scintillators in the muon system; no data from the SMT system is used for Level 1 trigger decisions. Except for the Level 1 muon triggers, which use data from the Level 1 tracking triggers, the Level 1 trigger does not perform any matching of objects from one detector subsystem with objects seen in another subsystem. This trigger lowers the initial $1.5 \mathrm{MHz}$ event rate down to around $1500 \mathrm{~Hz}$.

The Level 2 trigger is performed in two stages. The first step is a preprocessor step, where data from each detector subsystem is analyzed and sent on to the second stage, the Level 2 Global trigger. In the Level 2 Global trigger objects from various subsytems are matched to each other for the first time. This level of triggering lowers the Level 1 rate down to around $800 \mathrm{~Hz}$.

The final stage of triggering is the Level 3 system. Level 3 uses event reconstruction algorithms which have a level of sophistication close to what is used for the offline reconstruction. Event reconstruction at Level 3 allows for trigger decisions to be based on global variables such as missing momentum. This trigger reduces the output rate to tape to about 50 to $100 \mathrm{~Hz}$.

After beam has been injected into the Tevatron, the luminosity will fall as a function of time as the proton and anitproton beams become more diffuse and the particle density drops, as seen in Figure 12. As the luminosity falls, the trigger prescales are periodically lowered (meaning that more events are kept), which 


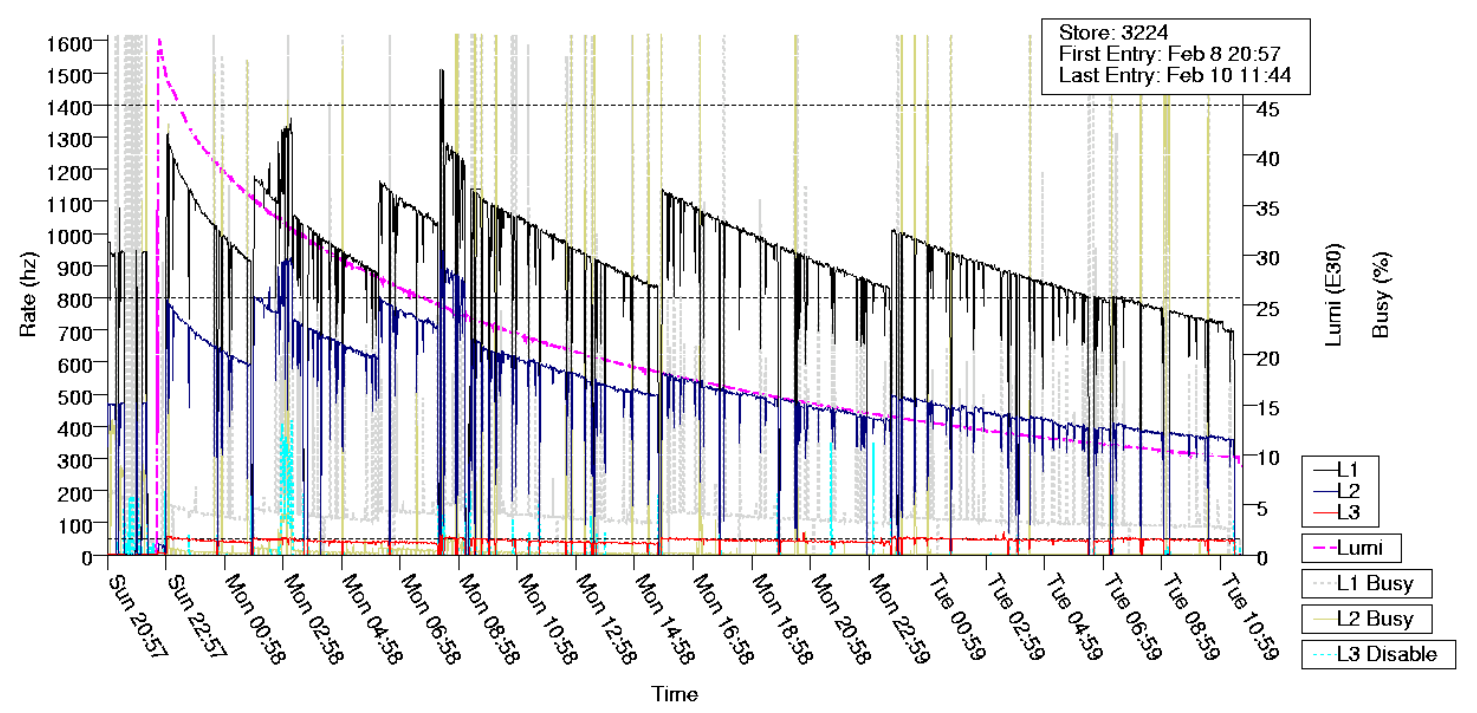

Figure 12: Trigger rates as a function of time for Store 3224.

results in the upward jumps seen in the trigger rates in Figure 12. In this way, the Level 3 output rate to tape can be maintained at a steady $50-100 \mathrm{~Hz}$ while the luminosity decays.

\subsubsection{Example: Electron trigger}

To further clarify the operation of the $\mathrm{D} \varnothing$ trigger system, we present here the example of the electron trigger. The work presented here was performed as part of the summer 2007 upgrade, and was necessary in order for the detector to continue operating efficiently at higher luminosities. This upgrade therefore does not affect the dataset used for this analysis, but will be relevant for all future analyses at DØ.

The upgraded DØ Level 1 electron trigger uses a "sliding window" algorithm. The sliding window algorithm searches for local maxima in the electromagnetic calorimeter in a window that is either $2 \mathrm{x} 1$ or $1 \mathrm{x} 2$ trigger towers in $\eta-\phi$. The previous Level 1 trigger system only used a single trigger tower, which lowered efficiency for electrons that land on the cracks between two trigger towers. 


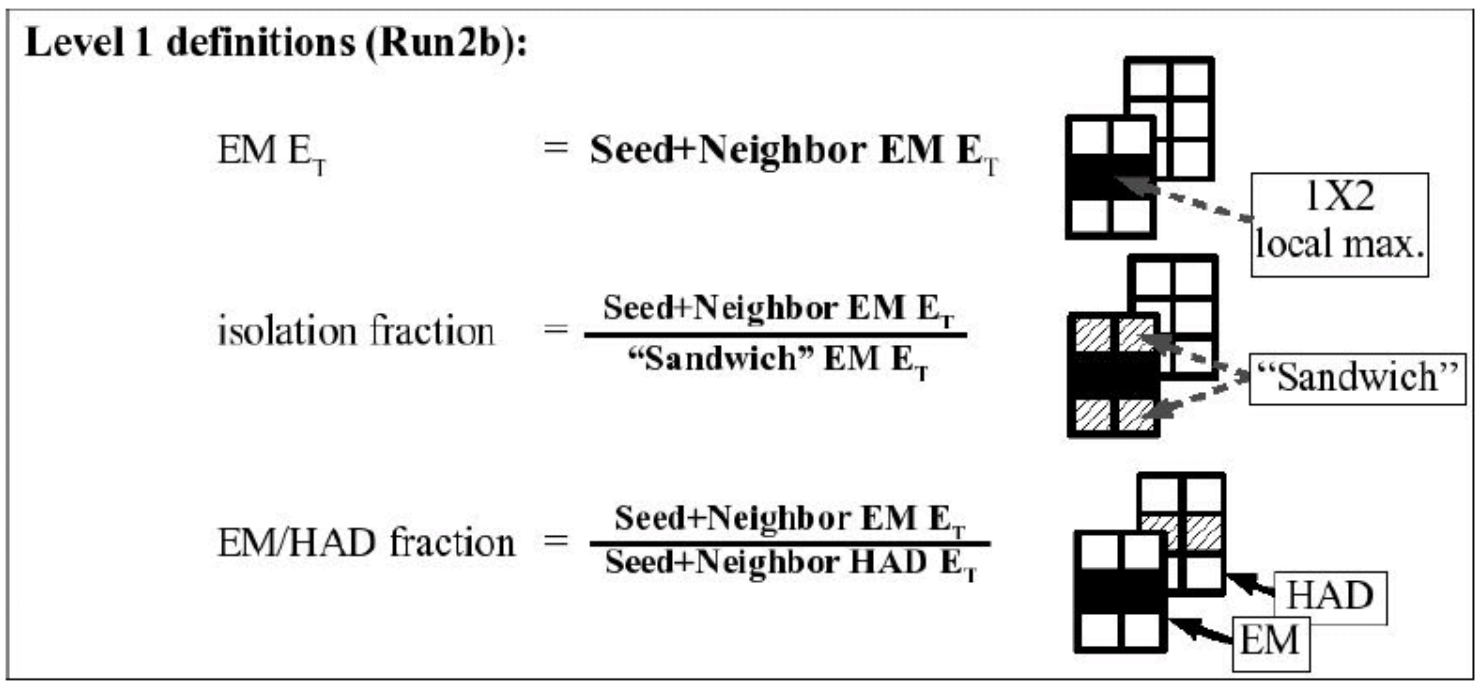

Figure 13: Definitions of the variables used for Level 1 electron trigger decisions. Each variable is defined as the black squares divided by the sum of the black and grey squares.

Once a local maximum is found, the total energy, electromagnetic fraction, and isolation variables are calculated as shown in Figure 13.

The updated Level 1 triggering is nearly as efficient as the previous Level 2 trigger, and therefore it became necessary to update the Level 2 trigger as well in order to have any substantial reduction in trigger rates at Level 2. It was decided that the Level 2 electron trigger should be improved through the use of a likelihood variable [40]. The variables chosen to construct the likelihood variable are shown in Figure 14. Once the variables were chosen, Probability Density Functions (PDFs) were constructed for both signal and background. Background PDFs were measured using a dataset where the only trigger requirement was a minimum bias trigger, which is a trigger that fires when any hard scattering process occurs. Such a data sample is almost completely QCD jets with only minimal contamination from real electrons. The signal PDFs were determined using $\mathrm{Z} \rightarrow$ ee Monte Carlo simulated events. 


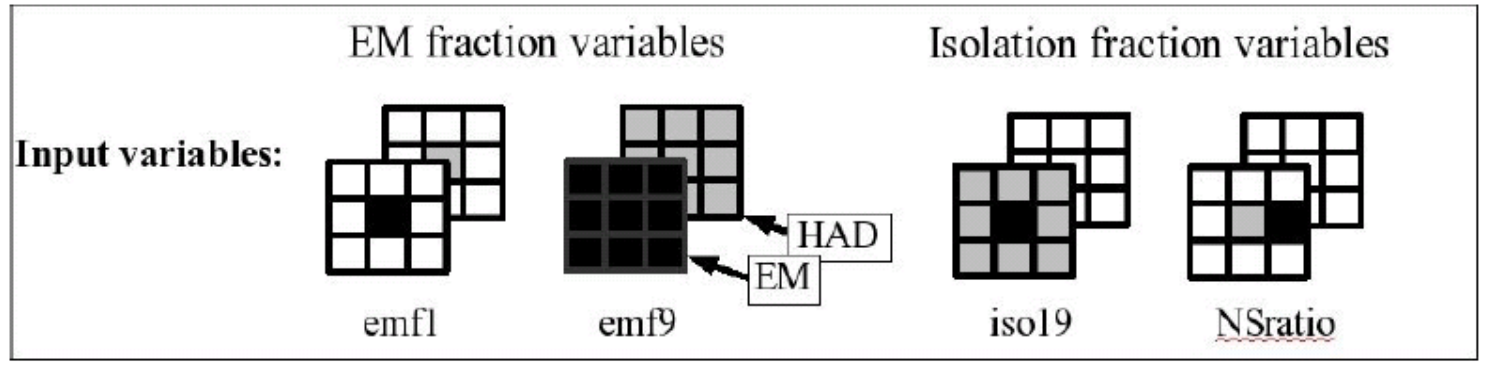

Figure 14: Definitions of the variables used for the new Level 2 likelihood variable. Each variable is defined as the black squares divided by the sum of the black and grey squares, except for the variable NSratio which is defined as black divided by grey.

Once the PDFs were determined, the signal to background probability ratio was evaluated for each variable and the product over all four variables was taken to get an overall signal:background ratio. In order to normalize a likelihood variable that lies between 0 and 1 , the likelihood was calculated from this ratio as $\frac{\text { Ratio }}{\text { Ratio }+1}$.

The Level 2 likelihood variable was extensively tested on an enhanced bias dataset designed for such trigger studies. The likelihood variable was tested on the so-called E1 and E2 trigger suites, which are described in Table 2. Each trigger suite refers to a set of Level 1 and Level 2 trigger requirements which can then be combined with a variety of trigger terms at Level 3. The purpose of this work was to determine whether the new likelihood variable could be used to reduce the trigger rates at Level 2 without adversely affecting the trigger efficiency for an offline reconstructed electron.

Table 3 shows the effect on the trigger rate for various Level 2 likelihood cuts. As can be seen, a cut of 0.2 on the likelihood has little to no effect on the trigger rate, indicating that such a cut is not substantially tighter than the Level 2 isolation cut already in place. Cuts of 0.8 or 0.95 result in substantial reductions in the trigger rate, especially for the E2 triggers. Figures 15 and 16 show the effects of the likelihood cut on the efficiency for a tight electron (as described in Section 4.1) to pass the trigger requirement. Cuts up to 0.5 on the likelihood variable have little 


\begin{tabular}{|l|l|l|}
\hline Trigger & Level 1 Requirement & Level 2 Requirement \\
\hline E1 & $E_{T}>19 \mathrm{GeV}$ & $\begin{array}{l}E_{T}>19 \mathrm{GeV} \text { and iso. }>0.2 \\
\text { OR } E_{T}>22 \mathrm{GeV}\end{array}$ \\
\hline E2 & $\begin{array}{l}E_{T}>16 \mathrm{GeV}, \text { and } \\
\text { iso. frac. and EM frac. }>8\end{array}$ & $E_{T}>16 \mathrm{GeV}$ and iso. $>0.2$ \\
\hline
\end{tabular}

Table 2: Trigger requirements at Level 1 and Level 2 for the E1 and E2 trigger suites. The Level 1 isolation fraction and EM fraction variables are defined in Figure 13. The Level 2 isolation fraction is defined as the EM energy of the seed tower plus the highest neighboring tower, divided by the total energy (EM plus hadronic) in a $3 \times 3$ grid around the seed tower.

impact on the turnons, while a cut of 0.95 clearly causes an unacceptably large loss in efficiency. A cut of 0.8 can be used to reduce the Level 2 trigger rates, but, as seen in these figures, it will cause some loss of efficiency in both the turnon part of the curve and the plateau at high energy.

\begin{tabular}{|r|r|r|}
\hline Likelihood Cut & E1 Suite Trigger Rate $(\mathrm{Hz})$ & E2 Suite Trigger Rate \\
\hline 0.00 & 200.42 & 146.48 \\
0.20 & 200.42 & 145.55 \\
0.50 & 195.77 & 134.85 \\
0.80 & 182.75 & 90.21 \\
0.95 & 169.26 & 39.53 \\
\hline
\end{tabular}

Table 3: Expected trigger rate for the E1 and E2 trigger suites for various cuts on the Level 2 likelihood variable. 


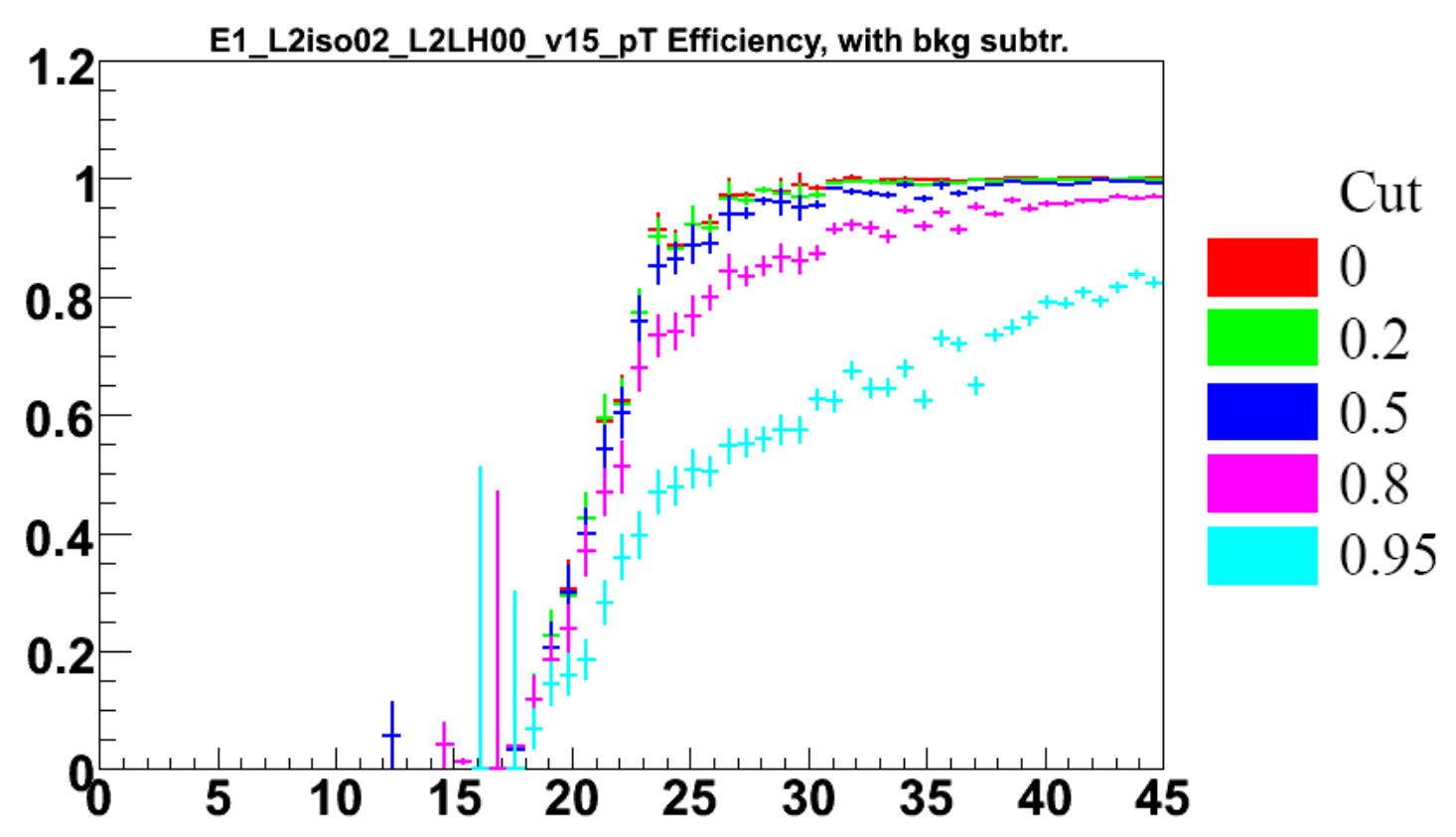

Figure 15: Turn on curves for the E1 trigger suite for various cuts on the Level 2 likelihood variable.

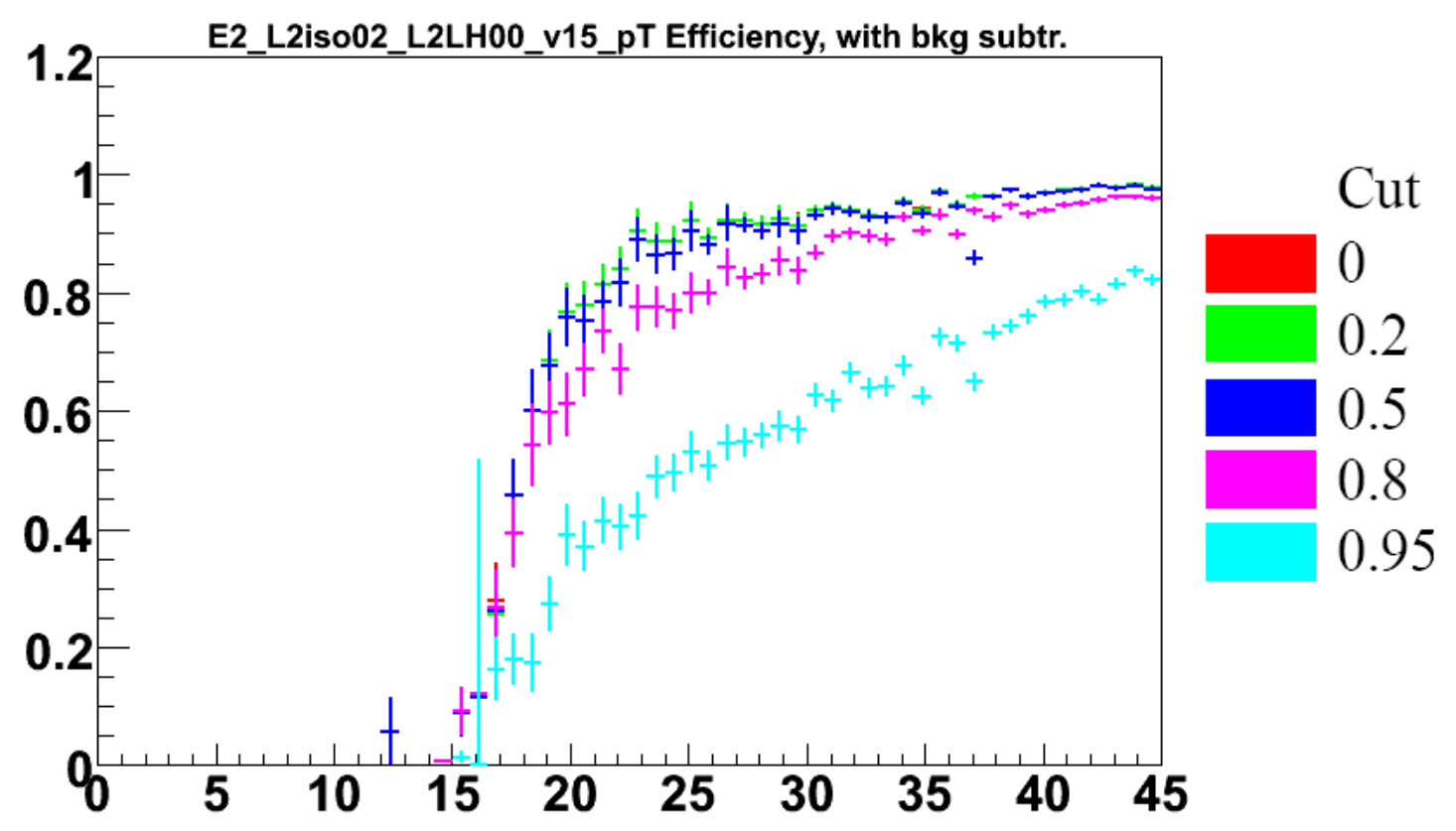

Figure 16: Turn on curves for the E2 trigger suite for various cuts on the Level 2 likelihood variable. 


\section{Object Identification}

Data from the detector consists of energy depositions in the calorimeter cells and hits in the muon and tracking systems. Such data must first be reconstructed into the physical objects in the event, e.g. electrons, photons, muons, and hadronic jets, before it will be useful for analysis. Event reconstruction is performed by the DØRECO program.

Particle reconstruction is done in three phases:

- Hit finding - Wire signals from the muon system PDTs and data from the SMT and CFT systems are combined and analyzed to determine the spatial position of hits in the muon and tracking system.

- Tracking and clustering - Individual hits in the muon and tracking systems are combined to find particle tracks and clusters of high-energy towers are identified in the calorimeter.

- Particle identification - Data from different detector components are combined to create physics objects, i.e., electromagnetic clusters in the calorimeter are matched to tracks in the tracking system, muons from the muon system are similarly track matched, etc.

This level of particle reconstruction provides very loose quality objects. The reconstruction efficiency is very close to $100 \%$ but there are substantial backgrounds that must be removed later through offline selection cuts.

\subsection{Electrons and Photons}

Electrons are reconstructed using a cone algorithm with a cone radius of 0.2 in $\eta-\phi$ space. To be considered as an electron (or photon) candidate at least $90 \%$ of the energy must be deposited in the electromagnetic layers of the calorimeter and at 
least $60 \%$ of the total cluster energy must reside in a single tower. These cuts remove most, though not all, of the hadronic jets. Electrons and photons are distinguished from each other by matching the calorimeter object to tracks within a $0.1 \times 0.1$ square in $\eta-\phi$ space; electrons will be track matched while photons can only be track matched if a charged particle happens to lie near the photon in $\eta-\phi$. The most important backgrounds to electron identification are:

- The $\pi^{0}$ meson decays electromagnetically, so if a $\pi^{0}$ lies next to a charged particle track it can fake an electron.

- Charged pions can form a very electromagnetic-like energy deposition in the calorimeter.

- A photon can interact with tracking system material and convert to an $e^{+} e^{-}$ pair.

The energy scale for electromagnetic objects receives corrections from two sources. The module that was used to gather the test beam data was not installed in the $\mathrm{D} \varnothing$ detector, and a correction factor must be applied to account for differences between the detector hardware and the test beam hardware. After this correction is performed, it is found that the position of the $\mathrm{Z}$ mass resonance does not agree with the precision measurements performed at LEP [41]. Therefore, electron energies must be scaled up in order to bring the observed Z mass into agreement with the LEP data.

The reconstruction level cuts described above include a substantial background from hadronic jets. In order to reduce this background a set of offline cuts are used to create a nearly pure sample of electrons [34]. These offline cuts are:

- The signature for an electron (or photon) in the calorimeter is an energy deposition in a very narrow cone which does not extend beyond the 
electromagnetic calorimeter layers. An isolation fraction variable has been defined as

$$
f_{\text {iso }}=\frac{E_{t o t}(0.4)-E_{E M}(0.2)}{E_{E M}(0.2)}
$$

where $E_{\text {tot }}(0.4)$ is the total energy deposited in all calorimeter layers (electromagnetic and hadronic) in a cone of radius 0.4 in $\eta-\phi$ centered on the electron candidate and $E_{E M}(0.2)$ is the energy deposited in just the electromagnetic calorimeter inside a cone of radius 0.2 . The isolation fraction is required to be less than 0.15 .

- The electron candidate must pass an H-matrix test which compares the candidate electron's shower shape to the expected shower shape of a typical electron [36]. This test is performed by first defining a matrix from a sample of Monte Carlo events:

$$
M_{i j}=\frac{1}{N} \sum_{n=1}^{N}\left(x_{i}^{n}-\bar{x}_{i}\right)\left(x_{j}^{n}-\bar{x}_{j}\right)
$$

where $N$ is the number of electrons in the Monte Carlo sample and the $x_{i}$ are a set of seven variables which characterize the shape of an electromagnetic shower. The variables used in the matrix are the fractional energy in each of the four electromagnetic calorimeter layers, the total electromagnetic energy, the width of the shower in $\phi$, and the z position of the vertex. The matrix $M_{i j}$ is evaluated as a function of the tower's $\eta$ (treating $+\eta$ and $-\eta$ equally) but the distribution is assumed to be flat in $\phi$ space. The agreement between a candidate electron's shower shape and the expected shower shape is determined by the $H$ matrix (the inverse of $M$ ): 


$$
\chi^{2}=\sum_{i, j=1}^{7}\left(x_{i}-\bar{x}_{i}\right) H_{i j}\left(x_{j}-\bar{x}_{j}\right)
$$

This $\chi^{2}$ is required to be less than 50 .

- The energy deposition in the calorimeter must be matched to a track observed in the central tracking system. This track matching not only requires the calorimeter object to be matched in $\eta-\phi$ space to the track, but the calorimeter energy must match the track's momentum within the expected uncertainties.

- A likelihood function is constructed based on the probability that the calorimeter cluster is a real, signal electron $\left(P_{s i g}\right)$ or a fake background $\left(P_{b k g}\right)$ :

$$
L=\frac{P_{s i g}}{P_{s i g}+P_{b k g}}
$$

The likelihood variables lies between 0 and 1 by construction, where a likelihood of 1 means the object is definitely an electron while 0 means it is a background object. The signal and background probabilities $P_{s i g}$ and $P_{b k g}$ are evaluated on data samples enriched in signal or background and are based on seven variables: the electromagnetic energy fraction, the $\chi^{2}$ of the H-matrix, the $\chi^{2}$ of the track match, the ratio $E \sin (\theta) / p_{T}$ (here $E$ is the energy measured by the calorimeter and $p_{T}$ is the transverse momentum measured by the tracker), the distance-of-closest-approach from the vertex, the number of tracks in a cone of $\mathrm{R}=0.05$, and the total $p_{T}$ of all tracks in a cone of radius $\mathrm{R}=0.4$.

Due to its large mass, the decay products from the top quark tend to be produced with a very high transverse momentum $p_{T}$ and low $\eta$ relative to most 
background processes that can occur. Because of this, the following cuts are applied to increase $t \bar{t}$ purity and reduce background:

- The electron must have a high transverse momentum, $p_{T}>15 \mathrm{GeV}$.

- The electron must be produced at an angle $\left|\eta^{\text {det }}\right|<1.1$, where $\eta^{\text {det }}$ (the "detector $\eta ")$ is the angle measured with respect to the center of the detector instead of the vertex position. Monte Carlo studies show that the acceptance for this cut is $73.4 \%$ for electrons coming from top quark decays and $49.8 \%$ for electrons in $\mathrm{Z} \rightarrow$ ee events.

\subsection{Muons}

Muons are detected from their reconstructed tracks in the muon system drift tubes ([35] - [38]). There are three stages to muon reconstruction. The first step is to determine the location of hits in the wire chambers, the second step is to combine hits into segments in the A, B, and C layers of the muon system, and finally these segments are combined to form a muon track.

The PDTs in the central muon system are capable of measuring both the drift time for ionized electrons to reach the central wire as well as the time it takes for the signal to travel down the wire. This allows for the determination of where the hit occurred along the wire. MDTs, by contrast, do not separate the drift time from the signal propagation time, so for the MDTs it is assumed that the hit occurred in the middle of the wire. Data from the muon scintillators can be used to assist in the determination of the timing of a hit and the hit position.

Muon track segments are found by forming line segments connecting two hits on different layers of drift tubes that are no more than $20 \mathrm{~cm}$ apart [39]. These segments are merged together into longer segments if they are consistent with a straight line. Track segments in the B and C layers, both of which are outside the 
toroidal magnetic field, are merged with each other to form a single segment. The $\chi^{2}$ for each segment is calculated, and only the segments with the lowest $\chi^{2}$ are kept in each octant.

Muon tracks are found by fitting the A layer segment to the segment through the BC layers [38]. The fitting algorithm must account for both the energy lost from bremsstralung in addition to the bending of the path from the toroid's magnetic field. The angle of deflection in the toroid gives a measurement of the muon's momentum. Muons used in this analysis, however, are matched to tracks in the tracking system, and the much more precise tracking system's momentum measurement is used instead of the muon system data. To match muon system tracks to tracking system tracks one must include effects from magnetic fields from the toroid and solenoid and also scattering effects in the calorimeter [43].

The most important muon backgrounds come from cosmic rays and hadronic jets which extend beyond the calorimeter. Hadronic jets are unlikely to extend past the calorimeter except in the region around $\eta \sim 1$, where due to the presence of the calorimeter's cryostat walls there is less material and thus fewer nuclear interaction lengths to absorb the jet's energy. To reduce the cosmic ray background the muon must pass timing cuts which ensure that the muon hits coincide with the beam crossing.

Additional cuts are applied offline to further purify the muon selection and remove backgrounds:

- The muon is required to have left a sufficient number of hits in the $\mathrm{A}, \mathrm{B}$, and C layer drift tubes and scintillators.

- The muon must have track segments in all three layers (A, B, and C) of drift tubes. 
- The muon must be matched in $\eta-\phi$ to a track in the tracking system. Several quality cuts are placed on this track. The tracking hit positions used in the fitting algorithm to construct the track must satisfy $\chi^{2} /$ ndof $<4$. Furthermore, the track must pass within a distance of $0.02 \mathrm{~cm}$ of the vertex if hits in the SMT tracking detector were used during track reconstruction. Tracks reconstructed with only data from the CFT tracker are less precise, and are only required to come within $0.2 \mathrm{~cm}$ of the vertex.

- Muons can be created through the decay of heavy quarks inside of a hadronic jet. To reject these muons we impose $\Delta R(\mu$, jet $)>0.5$.

- The ratio of the muon track's transverse momentum to the total transverse momentum in the calorimeter inside a hollow cone of inner radius 0.1 and outer radius 0.4 must be less than 0.06 . This cut helps to reduce the background of muons produced in hadronic decay where the hadronic jet was not properly identified as a jet.

- The ratio of the muon's track to the total momentum of all other tracks in a cone of radius $R=0.5$ must be less than 0.08 . Since jets have a high track multiplicity, this cut removes muons resulting from decay of particles inside a hadronic jet.

Due to the kinematics of the decay of a massive particle such as the top quark, the resulting muons tend to have a high transverse momentum together with a low $\eta$, so these kinematic cuts are imposed:

- The muon must have a large transverse momentum $p_{T}>15 \mathrm{GeV}$.

- The muon must be in the central region of the detector, $|\eta|<2$. When including the effects of this cut as well as the uninstrumented region on the 
lower part of the muon detector, the angular acceptance of muons is $87.6 \%$ for muons from top quark decays and $74.5 \%$ for muons in $\mathrm{Z} \rightarrow \mu \mu$ events.

\subsection{Tracks}

Tracks from electrically charged particles are reconstructed using data from the SMT and CFT channels. Each SMT channel is calibrated individually with gain and offset corrections. The initial analog signal is then digitized by an analog to digital converter (ADC). Particles passing through the tracker will most often activate multiple strips (SMT) or fibers (CFT) in each layer of the detector. Therefore, the first step of reconstruction is to form clusters of hits.

A cluster of hit strips in the SMT is defined as a set of consecutive strips which are above threshold (the threshold is set at 8 ADC counts), with the strips immediately before and immediately after below threshold [44]. The position of the centroid is defined as a weighted average:

$$
\mathrm{u}=\mathrm{u}_{1}+(\overline{\mathrm{n}}-1) p
$$

where $u_{1}$ is the position of the first strip in the cluster, $p$ is the pitch of the strip, and $\bar{n}$ is the weighted average of the number of ADC counts:

$$
\overline{\mathrm{n}}=\frac{\sum_{i} \mathrm{n}_{i} \mathrm{w}_{i}}{\sum_{i} \mathrm{w}_{i}}
$$

where $\mathrm{n}_{i}$ is the strip number and $\mathrm{w}_{i}$ is the number of ADC counts for the strip.

Clustering is defined similarly in the CFT layers. Clusters are defined as a set of consecutive fibers that are above threshold surrounded on either side by below threshold fibers. A single photoelectron corresponds to 15 ADC counts in the CFT axial layers and 7 ADC counts in the stereo layers. The threshold for the CFT 
fibers varies from 1.4 to 1.5 photoelectrons. The variation from fiber to fiber is necessary because some of the fibers have longer waveguides from the fiber to the photodetector than others, which results in greater signal attenuation [45]. For clusters in the CFT, the centroid of the cluster is just the midpoint of the cluster instead of a weighted average.

Once hit clusters have been formed the next step is to reconstruct track candidates from them ([46] - [47]). The search for track candidates begins in the innermost layers of the SMT and moves outward. Starting from an initial hit cluster, a second hit is found on a higher layer within 0.08 degrees in the axial direction from the first hit, followed by a third hit which forms a circle with the first two hits with a radius of greater than $30 \mathrm{~cm}$ (which represents an energy greater than 180 $\mathrm{MeV})$. Hits found in higher layers of the tracking system are included in the track candidate if they fall within the expected window. If two hits are found within the window, the second hit is used as the starting point for a new track candidate.

Once the track candidates have been determined, a set of cuts is applied to reject "fake" tracks - track candidates which, although formed from real hits, do not correspond to an actual particle in the detector. Track candidates are required to have an impact parameter less than $2.5 \mathrm{~cm}$ from the beamspot position, and the $\chi^{2}$ of the fit to the hit position must be less than 16. Also, restrictions are placed on the number of missed layers, and on the number of hits that the candidate shares with other track candidates.

To increase the efficiency of track reconstruction, tracks are also formed using CFT hits as the initial seed hits for the candidate. The procedure is similar to the procedure above for the SMT, except that in the end the track candidate is propagated backwards into the SMT to search for any additional hits on the inner silicon layers. 
In this analysis the second lepton from the tét pair decay is identified as an isolated track in the tracking system. In addition to quality cuts on the track we also need to apply isolation requirements to ensure that the track does not correspond to a particle in a hadronic jet. Therefore we impose the following offline cuts:

- The fitting of the track to the hit positions used to construct the track must satisfy $\chi^{2} /$ ndof $<4$.

- The distance of closest approach significance (the distance over its uncertainty) must be less than 2.5 .

- The track must be isolated from any jets, $\Delta \mathrm{R}$ (track, jet) $>0.5$.

- The track must not correspond to the lepton (either electron or muon) which has already been identified, so we require $\Delta \mathrm{R}$ (lepton, track) $>0.5$.

- The ratio of the track's transverse momentum to the total momentum of all other tracks within a cone of radius 0.5 must be less than 0.1 .

Top quark decay products usually have high transverse momentum and are relatively central, so we apply the kinematic cuts:

- High transverse momentum $p_{T}>15 \mathrm{GeV}$

- Central region of the detector $|\eta|<2.0$

Monte Carlo to data scale factors must be applied to correct the Monte Carlo efficiency to pass these cuts. A discussion of these scale factors and how they are calculated can be found in Appendix A. Since the scale factors have not been measured to arbitrarily high $p_{T}$, tracks are required to have $p_{T}<150 \mathrm{GeV}$. This cut is over $97 \%$ efficient and is necessary to remove obviously mis-measured tracks; tracks can be found with a $p_{T}$ as high as several $\mathrm{TeV}$. 


\subsection{Jets}

Due to quark confinement, the quarks and gluons created in a high energy collision will form into colorless hadrons through the strong interaction. These hadrons will have momenta that lie within a narrow cone of the originating particle and they are collectively referred to as a "jet". Jets are reconstructed from the calorimeter data using the improved legacy cone algorithm ([48], [49]).

The DØ calorimeter has some 55,000 channels. With such a large number of channels, there is a substantial probability for a random fluctuation to create a false signal. Therefore, before any processing of calorimeter data is done, a zero-suppression algorithm known as T42 is applied [52]. The T42 algorithm keeps all calorimeter cells that are more than $4 \sigma$ above zero, and any tower more than $2.5 \sigma$ above zero and immediately adjacent to a tower $4 \sigma$ above zero is also kept.

There are three steps in the cone algorithm used at DØ:

- Pre-clustering

- Clustering

- Splitting/Merging

The pre-clustering stage is seeded with all towers with a transverse energy greater than $0.5 \mathrm{GeV}$. A protojet is formed from each seed tower by doing a vector sum of all towers within a cone of radius $\mathrm{R}=0.3$ in $\eta-\phi$ space. This new vector is then used as the seed for another iteration, with all towers within a cone around it being summed up. This iterative process is continued until the center of the protojet is stable. After the pre-clustering stage is complete, all protojets within $10^{-6}$ of each other in $\eta-\phi$ are assumed to be the same protojet.

During the clustering stage, any two protojets that are within $\mathrm{R}=1.0$ of each other are vector added together and used as a seed for the iterative approach 
described above. If a new, stable protojet is found, this new protojet is kept and the original two are removed from the list; otherwise, the original protojets are kept.

The final stage is splitting/merging. In this stage, any protojet which does not share any towers with another protojet is automatically considered to be a jet. If any two protojets share less than $50 \%$ of their energy with each other, the shared energy is divided equally between the two, but if the shared energy is over $50 \%$, the two protojets are merged into a single protojet. If the protojets are either split or merged, the new protojets are used as seeds in the iterative process, and resulting stable protojets are considered to be a jet.

The measured jet energy is calibrated in order to reproduce as accurately as possible the energy of the orginating particle. The software package CAFIX ([50] [51]) is used to correct for calorimeter effects and equate the jet energy to the average total energy of the in-cone particles. After this correction is applied, it is found that the reconstructed energy is not equal to the originating particle's energy. This can occur for many reasons, such as gluon radiation outside of the reconstruction cone, dead material in the detector, non-linear effects, and of course noise. A correction is applied to account for these effects [53]:

$$
E_{\text {corr }}=\frac{E_{\text {measured }}-O}{R_{j} S}
$$

Here $O$ is an offset correction, $R_{j}$ is the calorimeter response, and $S$ is the fraction of energy which lies outside the reconstruction cone. The offset $O$ includes effects from electronic noise, uranium decay from the absorber plates, and extra interactions not related to the hard-scattering process being studied. This offset is measured by using a minimum bias trigger, which is a trigger that fires for any hard-scattering collision. The calorimeter response $R_{j}$ is evaluated using $\gamma+$ jet data events. Since the electromagnetic response is known much more precisely, the jet 
response can be determined from such events. Finally, the energy fraction out of cone $S$ can be determined by analyzing the shape of jets in data.

A number of quality cuts are placed on the jets in order to reject background:

- The electromagnetic fraction should not be too close to 1 , as this is very electron-like, but it is also not expected to be 0 either. The electromagnetic fraction is required to lie between 0.05 and 0.95 .

- No more than $40 \%$ of the total shower energy can lie in the coarse hadronic calorimeter layers. This is to prevent noise in the coarse hadronic calorimeter from being reconstructed as a jet.

- To reject fake jets that are caused by a hot calorimeter cell, the ratio of the most energetic cell to the next most energetic is required to be less than 10 .

- To similarly reject effects from hot towers, the jet is removed if more than $90 \%$ of the total energy is in one single tower.

- The jet is required to be confirmed by the Level 1 calorimeter trigger. This will be discussed further in Section 4.4.1.

\subsubsection{Upgraded Level 1 Confirmation Algorithm}

The signals from the calorimeter channels are split and copies are sent to the Level 1 trigger and to the precision readout which is used for final offline analysis. The trigger data and offline data are then digitized separately, which allows one to, for each offline reconstructed jet, "confirm" that the Level 1 trigger system recorded approximately the same amount of energy deposition. This is done by requiring the quantity

$$
\frac{\text { L1 energy }}{\text { Precision Energy } *(1-\mathrm{CH} \text { frac })} \text {, }
$$


to be greater than 0.4 in the central calorimeter and end calorimeters, or greater than 0.2 in the intercryostat region near $\eta=1$. Here $\mathrm{CH}$ frac is the fraction of energy in the coarse hadronic layers, which must be subtracted from the precision readout energy because the Level 1 trigger system does not include coarse hadronic data. The reason that this cut is looser in the intercryostat region is that there is less active material in the calorimeter for these $\eta$ values and therefore coarser resolution. The computation of the Level 1 trigger energy is a simple scalar sum of the individual trigger towers, while the precision readout uses the full vector sum. Both energy computations first apply a noise suppression algorithm before summing up the calorimeter towers. In the precision readout, this is the T42 algorithm discussed in Section 4.4. For the Level 1 trigger readout, the algorithm previously applied was simply to keep the 100 hottest towers in the event and zero out all remaining towers.

A number of improvements on the Level 1 Confirmation algorithm have been implemented. First, thresholds were set to determine which Level 1 towers to keep and use for calculating jet energy, instead of the simple 100 hottest towers approach used previously. By only using the hottest towers, the previous algorithm effectively set a higher tower threshold in high occupancy events (such as top pair events), than in quieter events with lower calorimeter occupancy. The chosen thresholds are shown in Table 4.

\begin{tabular}{|l|l|c|}
\hline$\eta$ region & Calorimeter layers & Threshold \\
\hline Central Calorimeter & Electromagnetic & $0.25 \mathrm{GeV}$ \\
Central Calorimeter & Hadronic & $0.50 \mathrm{GeV}$ \\
End Calorimeter & Electromagnetic & $0.25 \mathrm{GeV}$ \\
End Calorimeter & Hadronic & $0.50 \mathrm{GeV}$ \\
Intercryostat Detectors & & $0.50 \mathrm{GeV}$ \\
Massless Gap Detectors & & Not Used \\
\hline
\end{tabular}

Table 4: Threshold setting used for the updated Level 1 Confirmation algorithm. 
Separate thresholds were set for the electromagnetic and hadronic calorimeter layers. Therefore, it is possible for the electromagnetic layers to be above threshold and included in the jet's energy, while the hadronic energy is below threshold and therefore zeroed out, or vice versa. This fact can be particularly important since negative energy readings (noise) is allowed down to an energy of $-1.0 \mathrm{GeV}$, meaning that, for instance, a signal in the electromagnetic layers could be reduced by a negative fluctuation in the hadronic layers if one only considers the total tower energy.

The Intercryostat and Massless Gap detectors listed in Table 4 are detectors placed in between the central barrel calorimeter and the two end cap calorimeters. These detectors are designed to increase sensitivity for $\eta$ values around 0.8 to 1.4 where there is a significant amount of uninstrumented material in the croystat walls. The Massless Gap detectors in particular are quite noisy, and for the purposes of this study it turned out that better matching between the precision and trigger systems could be achieved by ignoring these detectors, so no threshold is set for them.

Figure 17 shows the number of towers that are kept in each event using the updated algorithm. In the majority of events, more than 100 towers are kept, and there is a tail out to over 400 towers per event. In very few events are less than 100 towers above threshold.

The updated algorithm was tested and compared with the original algorithm using a sample of clean dijet events. Events were required to be clear of known sources of calorimeter noise. The jets were required to be back-to-back in $\phi$, pass all of the jet quality criteria from Section 4.4 (except for the Level 1 confirmation cut, of course), and the jets were matched to hard tracks in the tracking system to further reduce fake jet backgrounds. Figure 18 shows plots of the variable

$$
\Delta p_{T}^{\text {rel }}=\frac{p_{T}^{\text {precision }}-p_{T}^{\text {Level } 1}}{p_{T}^{\text {precision }}}
$$




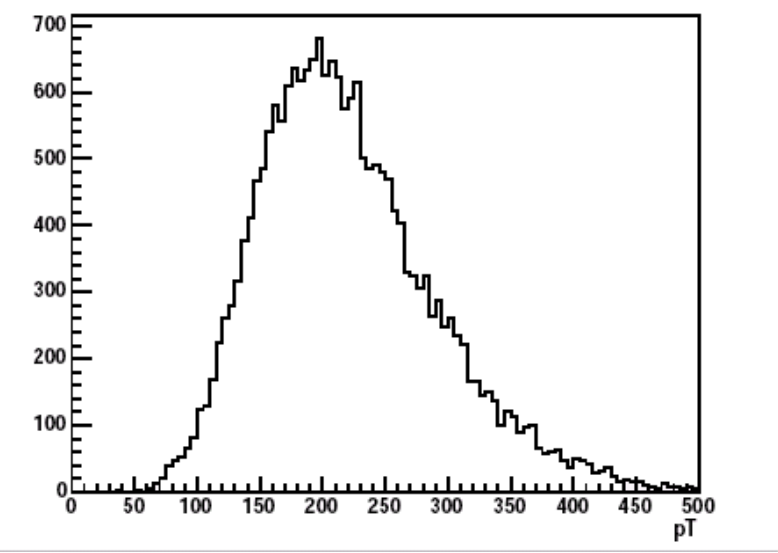

Figure 17: Number of towers above threshold using the updated Level 1 confirmation algorithm.

for both the original and improved algorithm. The updated algorithm's distribution has better resolution and the tails are substantially suppressed, which shows that the level of agreement between the precision readout energy and the Level 1 trigger energy measurement is significantly better. In particular, the high-end tail where the precision energy is greater than the Level 1 energy is highly suppressed, which is critical because this is the regime where the cut is being applied.

Further refinements to the Level 1 confirmation algorithm were considered during the course of this analysis. However, implementing these changes would have required substantial computing time to reprocess the dataset, and it was determined not to use computing resources towards this task. These corrections will be implemented in future datasets, but were not included in the dataset used by this analysis.

First, like any sampling calorimeter, the individual readout cells must be weighted by the relative amount of inactive to active material, to account for the energy deposited in the absorber material. Previously, the precision and Level 1 trigger readouts use different sets of weight factors which generally, but not always, 

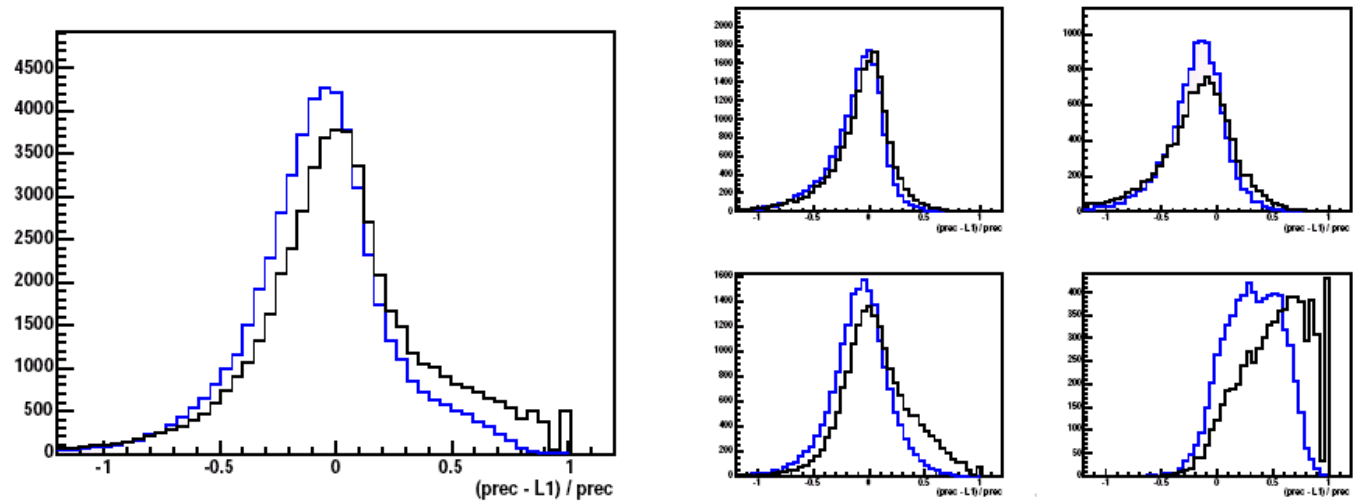

Figure 18: Comparison of the original and updated Level 1 confirmation algorithms. The original algorithm is the black curve and the updated algorithm is the blue curve.

have a ratio of approximately 1 . The actual ratio was found to vary from less than 0.5 up to about 2.5. These differences were accounted for by multiplying the precision readout cells by the ratio of Level 1 weight / precision weight.

The second correction was to use the jet's tower list to compute the Level 1 jet energy. Offline jets were reconstructed using the algorithm discussed in Section 4.4, while previously the Level 1 jets were reconstructed by using a simple cone algorithm with a radius of 0.5 in $\eta-\phi$. Since a $2 \times 2$ grid of precision towers is ganged together to form a single Level 1 trigger tower (the trigger uses coarser granularity for faster trigger decisions), an exact correspondence is impossible.

What has been implemented is to include a Level 1 trigger tower into the jet's total energy if any one of the four precision towers was a part of the offline jet.

The effect of the complete algorithm on the $\Delta p_{T}^{r e l}$ distribution is shown in Figure 19. From the distribution it is clear that these additional corrections help a great deal to improve the agreement between the Level 1 and precision readings and to suppress the tails in the $\Delta p_{T}^{r e l}$ distribution. 

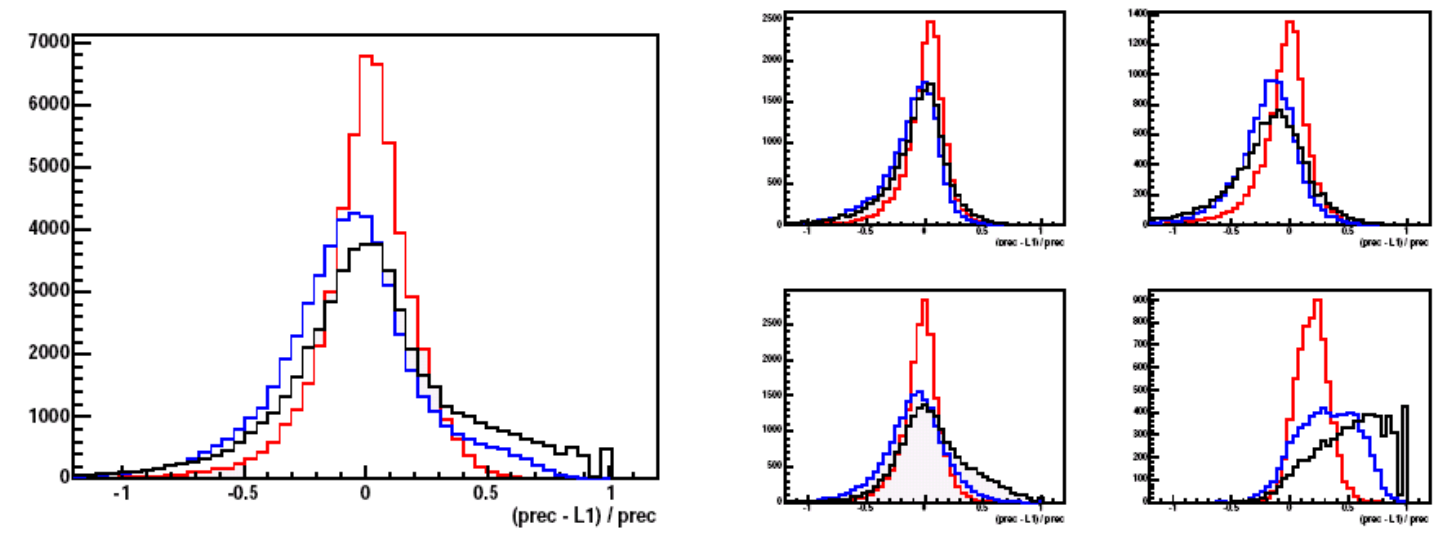

Figure 19: Comparison of various Level 1 confirmation algorithms. The original algorithm is the black curve and the implemented algorithm is the blue curve (these are the same as in Figure 18). The red curve shows the results of the additional corrections discussed in the text.

\subsection{Missing Transverse Momentum}

Neutrinos only interact through weak and gravitational interactions, and thus any neutrinos produced during a collision have a negligible chance of interacting with the detector material. Therefore, the presence of neutrinos can only be inferred from momentum conservation. In general, during any given collision the lab frame is not the center-of-mass frame - although the proton and antiproton carry a known momentum, the momentum fraction carried by the individual quarks or gluons which participated in the collision is not known. Since the momentum parallel to the beamline is unknown, it is impossible to use longitudinal momentum to search for neutrinos. Transverse momentum can be used, however - although the initial partons will have some momentum perpendicular to the beamline, this transverse momentum is very small in comparison to the center-of-mass energy and can therefore be taken to be zero. This allows one to use momentum conservation in the transverse plane to search for neutrinos. Transverse momentum carried off by neutrinos (or any other as-yet-unobserved long-lived weakly interacting particle) is 
known as missing transverse energy, or $\not_{T}$ (transverse energy is defined as $E_{T}=\sqrt{p_{T}^{2}+m^{2}}$. Neutrinos are essentially massless, so for them $\left.E_{T}=p_{T}\right)$.

The calculation of $E_{T}$ begins by summing over the calorimeter cells [42]

$$
\begin{aligned}
& \not_{T x}^{c a l}=-\sum_{i=1}^{N_{\text {cells }}} E_{x, i} \\
& E_{T y}^{\text {cal }}=-\sum_{i=1}^{N_{\text {cells }}} E_{y, i}
\end{aligned}
$$

This sum is taken after the T42 zero-suppression algorithm discussed in Section 4.4 is applied. Also, the coarse hadronic calorimeter cells which are not part of a jet are not included in the sum due to the large noise in these cells. The quantity $E_{T}{ }^{c a l}$ gives the missing momentum not observed by the calorimeter. This will include contributions from both neutrinos and muons, which only deposit a small fraction of their total energy in the calorimeter. $\not_{T}{ }^{c a l}$ is therefore corrected for the observed muons to give the desired quantity $\not_{T}$ :

$$
\begin{aligned}
& \not_{T x}=\not_{T}^{c a l}-\sum_{j=1}^{N_{\text {muons }}} p_{x, j} \\
& \#_{T y}=\#_{T y}^{c a l}-\sum_{j=1}^{N_{\text {muons }}} p_{y, j}
\end{aligned}
$$

The resolution of the $E_{T}$ calculation will of course be determined by the calorimeter resolution and also the muon resolution for events containing muons.

\subsection{Vertices}

In any given beam crossing it is possible to have multiple events, in particular it is common to have diffractive "soft scattering" events in addition to the "hard 
scattering" process of interest. These background interactions that occur are known as minimum bias interactions. It is therefore important to reconstruct the interaction vertex and to determine from which vertex each object in the event comes.

Vertices at $\mathrm{D} \varnothing$ are reconstructed using a two-pass approach. In the first pass, tracks satisfying very loose requirements are used to locate the beamspot and to identify loose quality vertices. The track selection requirements are tightened on the second pass, and the vertices are refit using the updated set of tracks. Furthermore, for each vertex, tracks with a contribution to the total $\chi^{2}$ greater than 10 are removed one by one until $\chi^{2} /$ ndof $<10$. Once the vertices have been identified, one vertex is selected as the "primary" vertex, i.e. the vertex assosciated with the hard-scatter process of interest. This is done by selecting the vertex that is least consistent with the hypothesis that it is a minimum bias interaction [59]. Hard-scatter collisions differ from minumum bias processes in that they have a larger number of tracks and a larger total transverse momentum.

After the primary vertex in any given beam crossing has been selected, further quality cuts are imposed on it in this analysis:

- The vertex must have at least 3 tracks associated with it.

- The z-position of the vertex must lie within $60 \mathrm{~cm}$ of the center of the detector. The distribution of collision vertices at $\mathrm{D} \varnothing$ is roughly a gaussian with a width $\sigma=30 \mathrm{~cm}$.

- Events in this analysis will contain both a lepton (either an electron or a muon) as well as an additional isolated track. Both the lepton's track and the isolated track are required to pass within $1 \mathrm{~cm}$ of the vertex in the $\mathrm{z}$-direction at their point of closest approach. 


\section{Event Selection}

The dilepton final state is characterized by two high $p_{T}$ leptons, two b quark jets, and large missing transverse energy. In the l+track channel, only one of the leptons is required to have been properly identified in the detector and the second lepton is identified by an isolated track in the tracking system. Two different search channels are discussed in this analysis: either an electron plus an isolated track or a muon plus an isolated track. The isolated track corresponds to an electron or a muon which was not identified using the standard lepton quality definitions. Because of this, it is not known what flavor of lepton produced the track, and therefore the electron+track channel will contain a mixture of electron-electron and electron-muon events, while the muon+track channel will consist of muon-muon as well as electron-muon events.

\subsection{Electron + Track Selection}

The electron+track analysis is performed using events from data and Monte Carlo simulations which satisfy the following conditions:

- Data events must have fired the electron+jet trigger, which requires one electron with $p_{T}>15 \mathrm{GeV}$ and a single jet with a $p_{T}$ of 20 to $30 \mathrm{GeV}$, depending on trigger version [62]. The effect of the trigger is simulated in Monte Carlo by applying the trigger's efficiency as a weight factor to each event. The trigger efficiency was parameterized as a function of $p_{T}$ and $\eta$.

- There must be one electron passing the electron selection outlined in Section 4.1. Furthermore, this electron must have fired the electron part of the electron+jet trigger.

- There must be at least one track passing the track selection of Section 4.3. 
- Since this analysis is designed to select events which would not have been included in a standard dilepton analysis where both leptons are fully identified, we veto events with a second identified lepton. A second electron veto has been imposed to make the electron+track selection orthogonal to the electron-electron channel, and a muon veto ensures that it is orthogonal to the electron-muon analysis [1].

- There must be at at least one jet passing jet identification criteria discussed in Section 4.4 .

- The leading jet must have $p_{T}>40 \mathrm{GeV}$. This high $p_{T}$ cut on the leading jet substantially reduces the background from the $\mathrm{Z}$ boson with only a few

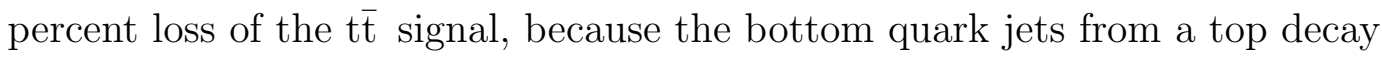
typically have high transverse momentum while $\mathrm{Z}$ bosons are rarely produced in conjunction with high energy jets.

- The $\#_{T}$ is required to be greater than $25 \mathrm{GeV}$. If the invariant mass of the combined electron+track momentum 4-vector lies between 70 and $110 \mathrm{GeV}$, this cut is increased to a $35 \mathrm{GeV}$ threshold. This cut is designed to reject the $\mathrm{Z} \rightarrow$ ee background, as $\mathrm{Z} \rightarrow$ ee events do not have a true $\mathbb{E}_{T}$.

- The $\#_{T}^{Z-f i t}$, to be discussed in Section 5.3, must be greater than $25 \mathrm{GeV}$. This is raised to $35 \mathrm{GeV}$ if the mass of electron+track system is between 70 and $110 \mathrm{GeV}$.

\subsection{Muon + Track Selection}

The muon+track analysis uses data and Monte Carlo events satisfying the following:

- Data events must pass a muon+jet trigger. This trigger requires one muon with a $p_{T}$ greater than $15 \mathrm{GeV}$, and at least one jet with a $p_{T}$ of 20 to 30 
$\mathrm{GeV}$, depending on the trigger version [62]. The trigger is simulated in Monte Carlo by applying the trigger efficiency as a weighting factor, and this efficiency was parameterized in $p_{T}$ and $\eta$.

- There must be a muon which passes the muon selection criteria discussed in Section 4.2, and this muon must have fired the muon part of the muon + jet trigger.

- There must be at least one track which satisfies the track selection cuts outlined in Section 4.3.

- To establish orthogonality with the muon-muon analysis, we reject events with a second identified muon, and we also veto events with identified electrons to ensure orthogonality with the electron-muon channel [1].

- The must be at least one jet passing the jet selection cuts from Section 4.4. Also, the highest $p_{T}$ jet must have a $p_{T}$ greater than $40 \mathrm{GeV}$. As in the electron case, this high momentum cut helps to remove the background from $\mathrm{Z}$

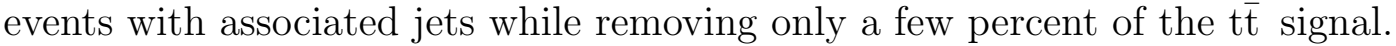

- The $E_{T}$ must be greater than $25 \mathrm{GeV}$, or $35 \mathrm{GeV}$ if the mass of the muon+track momentum lies between 70 and $110 \mathrm{GeV}$. This cut is designed to reject $\mathrm{Z} \rightarrow \mu \mu$ events.

- The $\not_{T}^{Z-f i t}$, to be discussed in Section 5.3, must be greater than $25 \mathrm{GeV}$, or 35 $\mathrm{GeV}$ if the mass of muon+track system is between 70 and $110 \mathrm{GeV}$.

- To reduce the background from $\mathrm{Z} \rightarrow \mu \mu$ events, we also impose a veto on events where the invariant mass of the identified muon plus any muon that passes a very loose selection cut is between 70 and $100 \mathrm{GeV}$. 


\subsection{Further Z background Rejection}

The dominant background in this analysis comes from $\mathrm{Z} \rightarrow \mathrm{ll}$ events with a large

fake $\not_{T}$. To reduce this background, a new variable $\not_{T}^{Z-f i t}$ is defined as follows:

- The momenta of the lepton and the track are rescaled such that the invariant mass of the track-lepton system is at the $\mathrm{Z}$ resonance of $91.2 \mathrm{GeV}$. This rescaling is done by simultaneously varying both the lepton and the track momenta (subject to the constraint that the invariant mass is $91.2 \mathrm{GeV}$ ) and minimizing the $\chi^{2}$ value, using the detector resolutions.

- The $\Delta p_{T}$ of the track and lepton is used as a correction to the $\not_{T}$ to get $E_{T}^{Z-f i t}$.

Tables 5 and 6 show the expected efficiency of the $E_{T}^{Z-f i t}$ cut for tét signal and $\mathrm{Z} \rightarrow \mathrm{ll}$ background after the $\not_{T}$ cut has already been applied. The $E_{T}^{Z-f i t}$ cut significantly reduces the $\mathrm{Z} \rightarrow \mathrm{ll}$ background, especially in the muon+track channel, with only a few percent loss in efficiency of signal tit events. The $E_{T}^{Z-f i t}$ distribution is shown in Figure 20 for $\mathrm{t} \overline{\mathrm{t}}$ and $\mathrm{Z} \rightarrow \mathrm{ll}$ samples before the $E_{T}$ and b-tagging cuts are applied.

\begin{tabular}{|lrrrrr|}
\hline MC Sample & Before & $\not_{T}^{Z-f i t}$ cut & After & $\not_{T}^{Z-f i t}$ cut & Efficiency \\
\hline $\mathrm{t} \overline{\mathrm{t}}$ & 18.83 & & 18.39 & $97.7 \%$ \\
$\mathrm{Z} \rightarrow \mathrm{ee}$ & 48.46 & & 37.30 & $77.0 \%$ \\
\hline
\end{tabular}

Table 5: Efficiency to pass $\not_{T}^{Z-f i t}$ cut in the electron+track channel. All numbers are before b-tagging has been applied. Number of events in the first column is the number of events after $\not_{T}$ has been applied but before $\mathbb{E}_{T}^{Z-f i t}$.

\subsection{Tagged samples}

The primary backgrounds for the lepton+track selection have a very low content of heavy flavor quark jets. Therefore, the backgrounds can be substantially suppressed 


\begin{tabular}{|c|c|c|c|}
\hline MC Sample & Before $E_{T}^{Z-f i t}$ cut & After $Z_{T}^{Z-f i t}$ cut & Efficiency \\
\hline $\mathrm{t} \overline{\mathrm{t}}$ & 14.65 & 13.96 & $95.3 \%$ \\
\hline $\mathrm{Z} \rightarrow \mu \mu$ & 153.20 & 50.08 & $32.7 \%$ \\
\hline
\end{tabular}

Table 6: Efficiency to pass $E_{T}^{Z-f i t}$ cut in the muon+track channel. All numbers are before b-tagging has been applied. Number of events in the first column is the number of events after $E_{T}$ has been applied but before $\#_{T}^{Z-f i t}$.

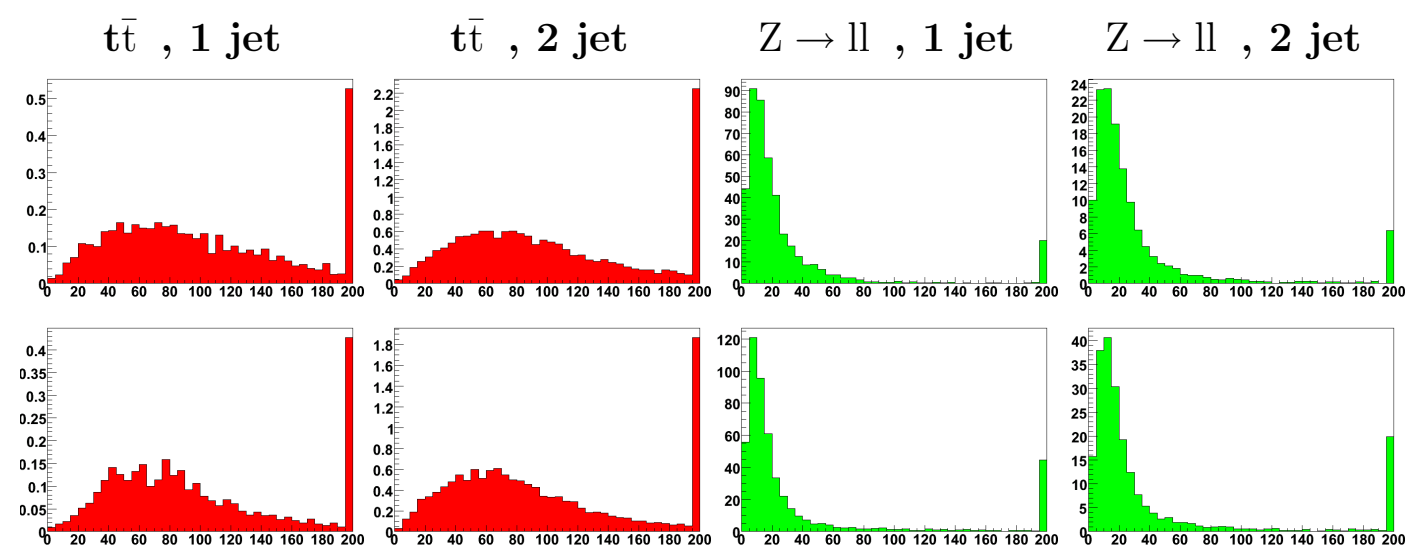

Figure 20: $\quad \not_{T}^{Z-f i t}$ distribution $($ in $\mathrm{GeV}$ ) for $\mathrm{t} \overline{\mathrm{t}}$ and $\mathrm{Z} \rightarrow \mathrm{ll}$ samples. All selection cuts have been applied except for $E_{T}, \quad E_{T}^{Z-f i t}$ and b-tagging. Top row is the electron+track channel and bottom row is the muon+track channel.

by requiring at least one of the jets to contain a b quark. In this anaylsis, the efficiency for a tét to dilepton event with two reconstructed jets to be identified as having at least one of those jets from a b quark is almost $70 \%$. This number falls to $50 \%$ if only one of the jets was reconstructed.

In addition to the electron+track cuts outlined in Section 5.1 and the muon+track selection from Section 5.2, we add the additional requirement that at least one jet must pass the medium cut of the $\mathrm{D} \varnothing \mathrm{NN}$ tagger $(\mathrm{NN}$ output $>0.65$ ) [60]. MC events are not tagged directly but instead are weighted according to the probability that they would pass the tagging cut:

$$
P=1-\prod_{i=1}^{N j e t s}\left(1-\epsilon_{i}\right)
$$


where $\epsilon_{i}$ is the probability for a jet to be tagged. The probability for a jet to be tagged is broken down into the taggability and the tag rate function; taggability is the efficiency for a jet to have tracks in the tracking system and includes effects from tracking inefficiency and calorimeter noise, while the tag rate function is the efficiency for a taggable jet to be tagged. The taggability of light parton jets is measured in data. Heavy flavor jets have greater track multiplicity and thus higher taggability, so the ratio of the taggability of heavy (b or c) jets to light flavor jets is estimated from Monte Carlo and verified on a data sample enriched in heavy flavor jets. The tag rate functions are measured with data using the System8 formalism, which constructs a system of 8 equations and 8 unknowns using two taggers and two data samples with different b quark content. The NN output is displayed in Figure 21. A detailed description of the $\mathrm{D} \varnothing \mathrm{NN}$ tagger and the measurement of the taggability and tag rate function can be found in [60]. 


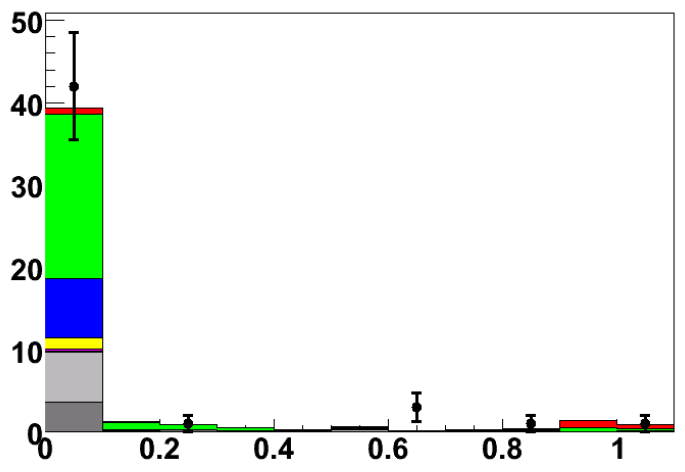

(a)

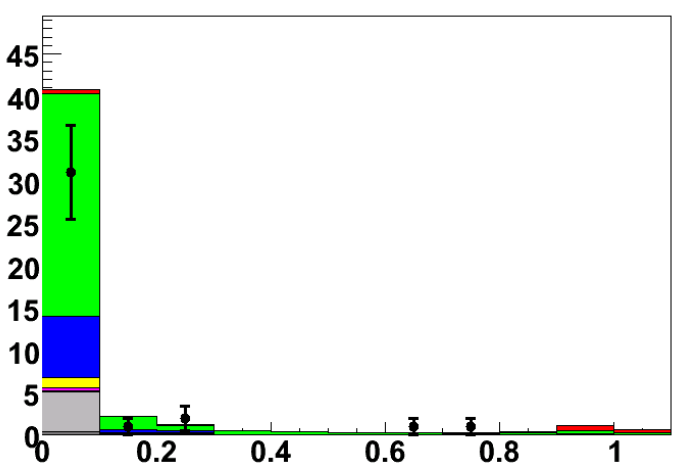

(c)

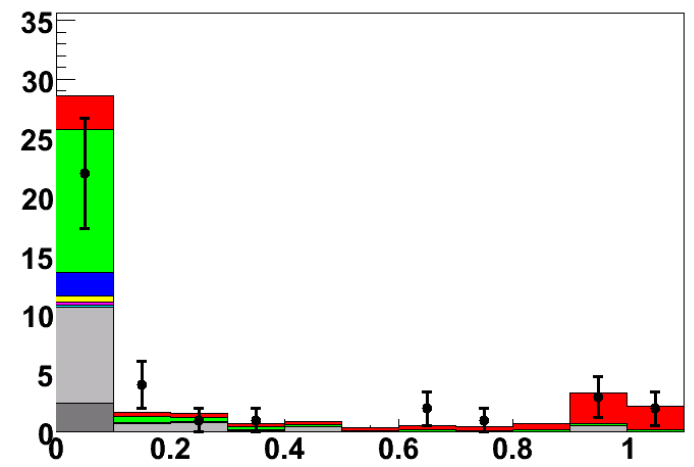

(b)

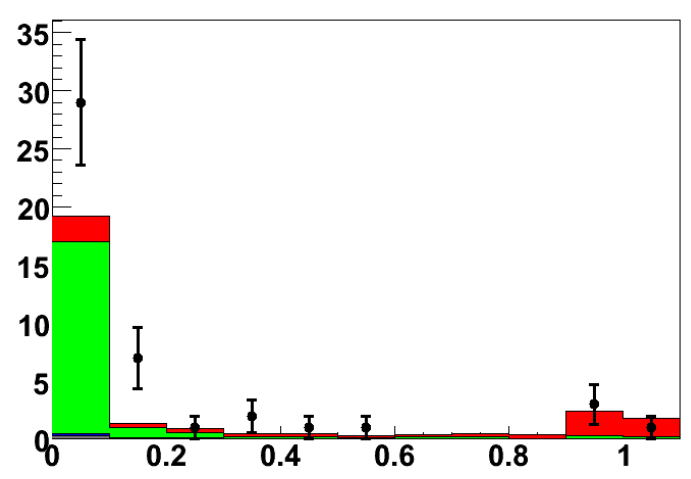

(d)

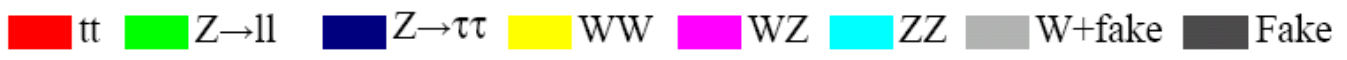

Figure 21: Distribution of the output variable from the DØ Neural Network tagger. (a) is the electron+track one jet bin, (b) is the electron+track two jet bin, (c) is the muon+track one jet bin, and (d) is the muon+track two jet bin. 


\section{Event Samples}

\subsection{Dataset}

The dataset used for this analysis is the full Run 2 a dataset, which was collected from April 2002 to March 2006. The recorded luminosity for this dataset is 1036 $\mathrm{pb}^{-1}$ in the electron+track channel and $994 \mathrm{pb}^{-1}$ in the muon+track channel.

\subsection{Signal Simulation}

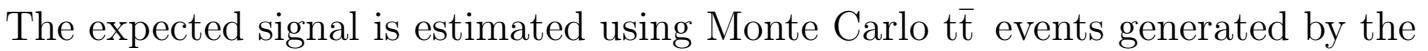
Alpgen event generator [63], which uses leading order (LO) matrix elements. Events are then processed by Pythia [64] for the hadronization, fragmentation and short-lived particle decay. Samples are generated with 0,1 , or 2 or more additional light quark or gluon jets.

\subsection{Diboson}

The diboson backgrounds (WW, WZ, and ZZ) are simulated using Monte Carlo events produced by the Pythia event generator. Pythia is a leading order event generator, so the cross sections are normalized with a $\mathrm{K}$ factor to bring it up to the next-to-leading order (NLO) cross section. The WW sample is normalized to the NLO cross section of $12.0 \mathrm{pb}$, WZ sample is normalized to $3.68 \mathrm{pb}$, and ZZ to 1.42

pb [61]. The K-factor between LO and NLO is approximately 1.4 [65], and we use a generous systematic uncertainty of \pm 0.4 on this $\mathrm{K}$ factor.

\subsection{Z boson}

The $\mathrm{Z} \rightarrow$ ee background in the electron+track channel and the $\mathrm{Z} \rightarrow \mu \mu$ background in the muon+track channel are estimated using the Alpgen event generator in a 
manner similar to the discussion for t $\overline{\mathrm{t}}$ events in Section $6.2 . \mathrm{Z} \rightarrow$ ee and $\mathrm{Z} \rightarrow \mu \mu$ events are generated in $0,1,2$, or 3 extra light parton bins and contributions with heavy flavors $b \bar{b}$ and $c \bar{c}$ are generated separately with 0,1 , or 2 light partons. There is also a $\mathrm{Z} p_{T}$ reweighting that must be applied to correct the Alpgen $\mathrm{Z} p_{T}$ spectrum to what is observed in data.

After all other Monte Carlo weights have been applied, including the $\mathrm{Z} p_{T}$ reweighting, a $\mathrm{K}$ factor for the $\mathrm{Z}$ background is found by first defining a nearly pure sample of $\mathrm{Z}$ events. This sample is selected using the same sample discussed in Sections 5.1 (electron+track) and 5.2 (muon+track) except that both the $E_{T}$ and $Z_{T}^{Z-f i t}$ cuts are reversed:

- $E_{T}<25 \mathrm{GeV}$ or $\#_{T}<35 \mathrm{GeV}$ if track-lepton mass is $70-110 \mathrm{GeV}$

- $E_{T}^{Z-f i t}<25 \mathrm{GeV}$ or $\#_{T}^{Z-f i t}<35 \mathrm{GeV}$ if track-lepton mass is $70-110 \mathrm{GeV}$

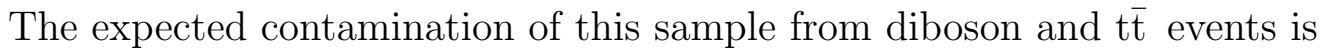
estimated from Monte Carlo, and the estimate from fake, instrumental backgrounds is determined from the matrix method, which will be discussed in Section 6.6. The total is subtracted from the number of data events found, and the result is divided by the number of $\mathrm{Z} \rightarrow$ ee $($ or $\mathrm{Z} \rightarrow \mu \mu$ ) events predicted by Monte Carlo simulation. The number of events in the $\mathrm{Z}$ sample and the $\mathrm{K}$ factors are shown in Table 7 for the electron+track channel and Table 8 for the muon+track channel. The $\mathrm{Z}$ resonance peak is displayed in Figure 22 for the electron+track channel and Figure 23 for the muon+track channel.

\begin{tabular}{|rrr|rrrrrr|r|r|}
\hline & $\mathrm{Z} \rightarrow \mathrm{ee}$ & $\mathrm{Z} \rightarrow \tau \tau$ & $\mathrm{t} \overline{\mathrm{t}}$ & $\mathrm{WW}$ & $\mathrm{WZ}$ & $\mathrm{ZZ}$ & $\mathrm{W}+$ fake & $\mathrm{QCD}$ & Data & $K_{Z}$ \\
\hline 1 jet & 270.19 & 0.73 & 0.10 & 0.10 & 0.70 & 0.63 & 0.71 & 1.33 & 308 & 1.12 \\
2 jet & 78.53 & 0.24 & 0.39 & 0.02 & 1.30 & 1.31 & 0.58 & 0.43 & 88 & 1.07 \\
\hline
\end{tabular}

Table 7: Number of expected and observed events in the $\mathrm{Z}$ sample in the electron+track channel, used to calculate $K_{Z}$. 


\begin{tabular}{|rrr|rrrrrr|r|r|}
\hline & $\mathrm{Z} \rightarrow \mu \mu$ & $\mathrm{Z} \rightarrow \tau \tau$ & $\mathrm{t} \overline{\mathrm{t}}$ & $\mathrm{WW}$ & $\mathrm{WZ}$ & $\mathrm{ZZ}$ & $\mathrm{W}+$ fake & $\mathrm{QCD}$ & Data & $K_{Z}$ \\
\hline 1 jet & 308.22 & 0.84 & 0.11 & 0.09 & 0.89 & 0.77 & 0.55 & 0.18 & 324 & 1.04 \\
2 jet & 106.95 & 0.52 & 0.63 & 0.04 & 1.55 & 1.86 & -0.09 & 0.04 & 131 & 1.18 \\
\hline
\end{tabular}

Table 8: Number of expected and observed events in the $\mathrm{Z}$ sample in the muon+track channel, used to calculate $K_{Z}$.

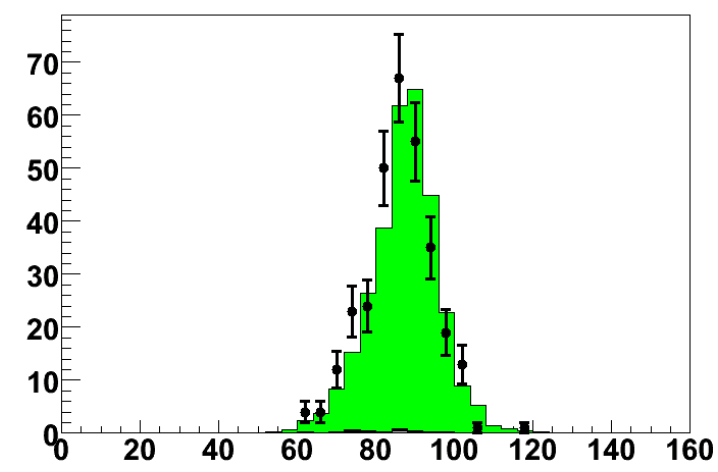

(a)

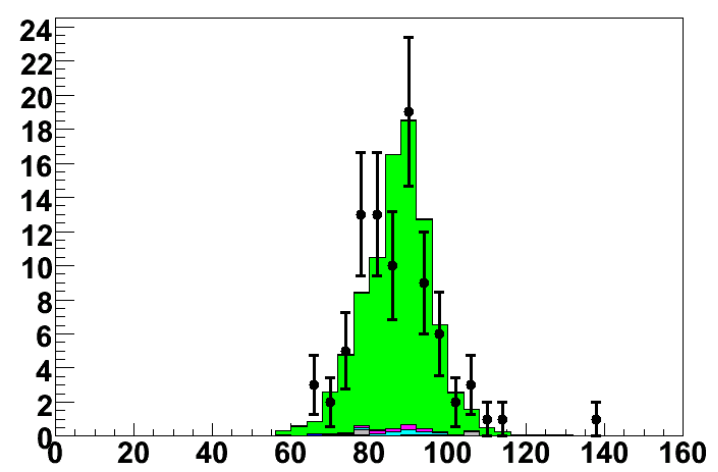

(b)

$\square$ tt $\quad$ Z $\rightarrow$ ee $\square Z \rightarrow \tau \tau \quad$ WW $\quad$ WZ $\quad$ ZZ $\quad$ W+Fake $\quad$ Fake+Fake

Figure 22: Electron+track mass distributions $\left(\left(E_{e}+E_{t}\right)^{2}-\left(\vec{p}_{e}+\vec{p}_{t}\right)^{2}\right.$, where $E_{e}$ and $\vec{p}_{e}$ are the energy and momentum of the electron and $E_{t}$ and $\vec{p}_{t}$ are the energy and momentum of the track) in $\mathrm{GeV}$ for the low $E_{T}$ sample used for $\mathrm{Z}$ boson $\mathrm{K}$ factor determination. (a) is the one jet bin and (b) is the two jet bin. The $K_{Z}$ factor has been applied in these plots, such that the Monte Carlo distribution is normalized to the data.

An additional $\mathrm{K}$ factor must be applied to the $\mathrm{Z}+$ heavy flavor $(\mathrm{Z}+c \bar{c}$ or $\mathrm{Z}+b \bar{b})$ Monte Carlo. The relative heavy flavor to light flavor $\mathrm{K}$ factor was measured on the sample of events with all of the analysis cuts of Sections 5.1 and 5.2 as well as the b-tagging requirement from Section 5.4 but with the $Z_{T}$ and $E_{T}^{Z-f i t}$ cuts reversed. This sample is the b-tagged version of the sample that was used to calculate $K_{Z}$ above. The number of expected and observed events in this sample is shown in Tables 9-10. The Z boson resonance peaks are shown in Figure 24.

The number of expected events is a function of the heavy flavor to light flavor $\mathrm{K}$ factor:

$$
\tilde{N}_{i}=\mathrm{K}_{\mathrm{HF}} N_{i}^{Z+H F}+N_{i}^{\text {other }}
$$




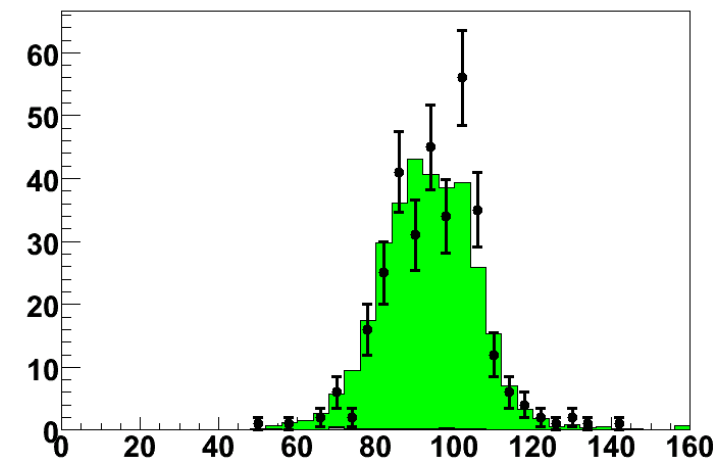

(a)

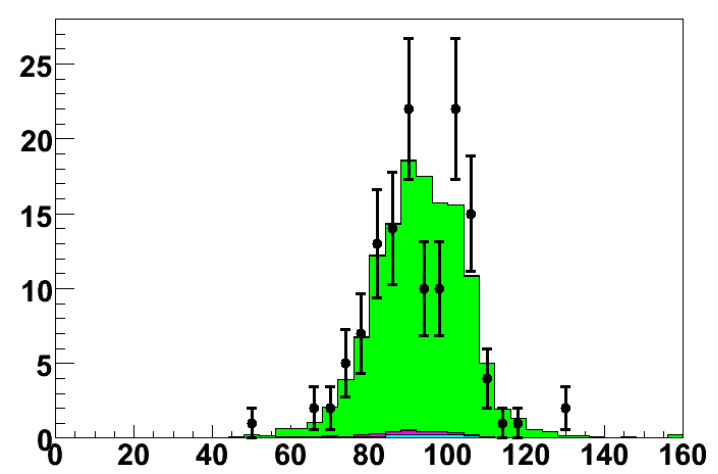

(b)

$\square$ tt $\quad \mathrm{Z} \rightarrow \mu \mu \quad \mathrm{Z} \rightarrow \tau \tau \quad$ WW $\quad$ WZ $\quad$ ZZ $\quad$ W+Fake $\quad$ Fake+Fake

Figure 23: Muon+track mass distributions $\left(\left(E_{\mu}+E_{t}\right)^{2}-\left(\vec{p}_{\mu}+\vec{p}_{t}\right)^{2}\right.$, where $E_{\mu}$ and $\vec{p}_{\mu}$ are the energy and momentum of the muon and $E_{t}$ and $\vec{p}_{t}$ are the energy and momentum of the track) in $\mathrm{GeV}$ for the low $E_{T}$ sample used for $\mathrm{Z}$ boson $\mathrm{K}$ factor determination. (a) is the one jet bin and (b) is the two jet bin. The $K_{Z}$ factor has been applied in these plots, such that the Monte Carlo distribution is normalized to the data.

\begin{tabular}{|rr|rrrrrrr|r|}
\hline & Z+h.f. & Z+l.f. & $\mathrm{t} \overline{\mathrm{t}}$ & WW & WZ & ZZ & W+fake & QCD & Data \\
\hline 1 jet & 3.81 & 2.39 & 0.05 & 0.00 & 0.03 & 0.09 & 0.01 & 0.05 & 5 \\
2 jet & 2.65 & 1.13 & 0.27 & 0.00 & 0.08 & 0.24 & 0.01 & 0.07 & 5 \\
\hline
\end{tabular}

Table 9: Number of expected and observed events in the b-tagged Z sample in the electron+track channel. These results are used to calculate a relative heavy flavor to light flavor $\mathrm{K}$ factor. Z+heavy flavor and $\mathrm{Z}+$ light flavor include contributions from both $\mathrm{Z} \rightarrow$ ee and $\mathrm{Z} \rightarrow \tau \tau$.

where the index i runs over both channels and jet multiplicity bins, $\mathrm{K}_{\mathrm{HF}}$ is the relative heavy flavor to light flavor scale factor, and $N_{i}^{\text {other }}$ is all other contributions to the expected number of events, including $\mathrm{Z}+$ light flavor, $\mathrm{t} \overline{\mathrm{t}}$, diboson, $\mathrm{W}+$ fake, and QCD. Here both the $\mathrm{Z}+$ light flavor and $\mathrm{Z}+$ heavy flavor samples already have the light flavor $K_{Z}$ factor from Tables 7 and 8 applied. The likelihood is then the product of the Poisson distributions:

$$
\mathscr{L}\left(\mathrm{K}_{\mathrm{HF}},\left[N_{i}^{\text {obs }}, N_{i}^{Z+H F}, N_{i}^{\text {other }}\right]\right)=\prod_{i=1}^{4} \frac{\tilde{N}_{i}^{N_{i}^{o b s}}}{N_{i}^{\text {obs }}} e^{-\tilde{N}_{i}}
$$

$\mathrm{K}_{\mathrm{HF}}$ is found by maximizing this likelihood. The result is 


\begin{tabular}{|rr|rrrrrrr|r|}
\hline & Z+h.f. & Z+l.f. & $\mathrm{t} \overline{\mathrm{t}}$ & $\mathrm{WW}$ & $\mathrm{WZ}$ & $\mathrm{ZZ}$ & $\mathrm{W}+$ fake & QCD & Data \\
\hline 1 jet & 4.50 & 2.68 & 0.06 & 0.00 & 0.03 & 0.10 & 0.00 & 0.00 & 10 \\
2 jet & 4.51 & 1.79 & 0.45 & 0.00 & 0.11 & 0.31 & 0.00 & 0.00 & 9 \\
\hline
\end{tabular}

Table 10: Number of expected and observed events in the b-tagged Z sample in the muon+track channel. These results are used to calculate a relative heavy flavor to light flavor $\mathrm{K}$ factor. $\mathrm{Z}+$ heavy flavor and $\mathrm{Z}+$ light flavor include contributions from both $\mathrm{Z} \rightarrow \mu \mu$ and $\mathrm{Z} \rightarrow \tau \tau$.

$$
\mathrm{K}_{\mathrm{HF}}=1.24_{-0.33}^{+0.37}
$$

\subsection{Z $\rightarrow \tau \tau$}

The $\mathrm{Z} \rightarrow \tau \tau$ background is estimated using the Alpgen event generator using a technique identical to the discussion for $\mathrm{Z} \rightarrow \mathrm{ll}$ events in Section 6.4. The same $\mathrm{K}$ factors that are used for the $\mathrm{Z} \rightarrow$ ee (electron+track) or $\mathrm{Z} \rightarrow \mu \mu$ (muon+track) background (see Section 6.4) are applied to the $\mathrm{Z} \rightarrow \tau \tau$ Monte Carlo.

\subsection{Fake Lepton Background Estimation}

The estimate for the background from fake leptons and/or tracks is made by solving a $4 \times 4$ matrix, as explained below. Here a "real track" is a track from a lepton and a "fake track" is a track from any other source. A track from a misidentified jet would still be called "fake" in this context even if it corresponds to an actual, observed particle. There are three possible sources of fake events which must be estimated:

- The identified lepton is real, but a QCD jet fakes the track $\left(N_{R L, F T}\right)$.

- A QCD jet fakes the identified lepton, but the track comes from a real lepton $\left(N_{F L, R T}\right)$.

- A QCD multijet event has a fake identified lepton and a fake track $\left(N_{F L, F T}\right)$. 


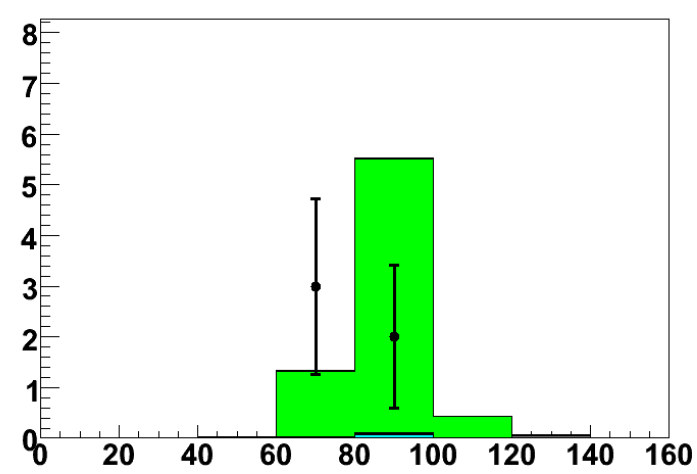

(a)

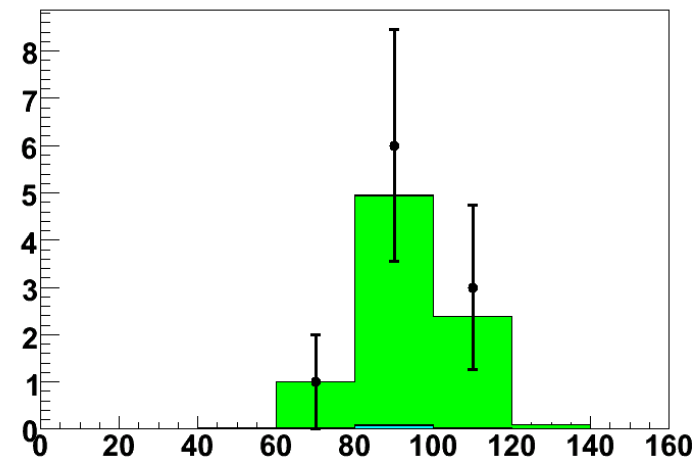

(c)

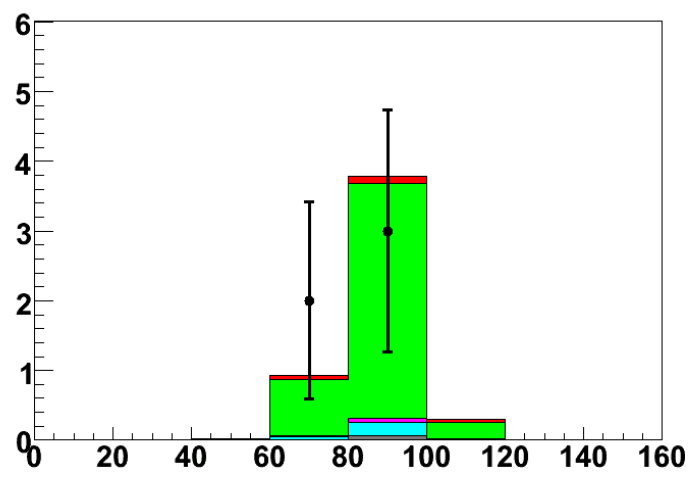

(b)

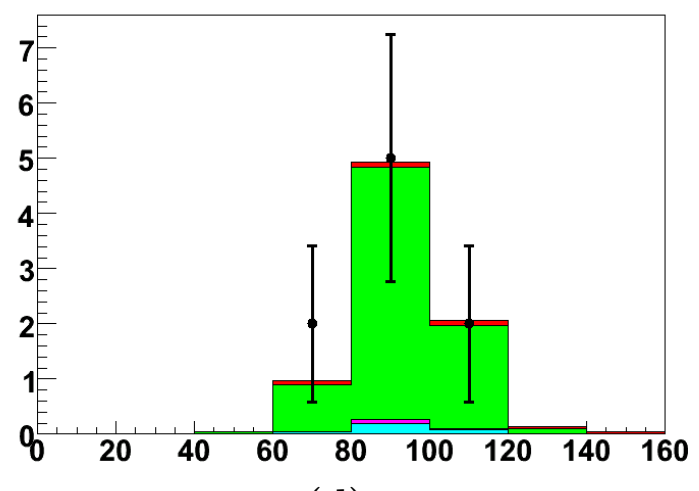

(d)

$\square$ tt $\quad \mathrm{Z} \rightarrow \mathrm{ll} \quad \square \mathrm{Z} \rightarrow \tau \tau \quad \mathrm{WW}$

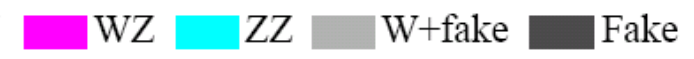

Figure 24: Lepton+track mass distributions $\left(\left(E_{l e p}+E_{t}\right)^{2}-\left(\vec{p}_{l e p}+\vec{p}_{t}\right)^{2}\right.$, where $E_{l e p}$ and $\vec{p}_{l e p}$ are the energy and momentum of the lepton and $E_{t}$ and $\vec{p}_{t}$ are the energy and momentum of the track) in $\mathrm{GeV}$ for the low $\not_{T}$ tagged sample used to determine the heavy flavor $\mathrm{K}$ factor. (a) is the electron+track channel one jet bin, (b) is the electron+track channel two jet bin, (c) is the muon+track one jet bin, and (d) is the muon+track channel two jet bin. All K factors have been applied for these plots.

In the matrix method one defines a looser set of selection requirements on the identified lepton and the isolated track. Events selected with the loose selection requirements are enriched in background from QCD jets faking the lepton and/or track. In this analysis, loose objects are defined as follows:

- Loose electrons do not have any cut on the electron likelihood variable. The likelihood variable is designed to distinguish electrons from QCD jets. 
- Loose muons do not have any calorimeter isolation or track isolation requirements.

- Loose tracks have a weaker track isolation requirement.

The loose lepton, loose track $\left(N_{L L, L T}\right)$ sample has three subsamples created by tightening the lepton cut $\left(N_{T L, L T}\right)$, the track cut $\left(N_{L L, T T}\right)$ or both $\left(N_{T L, T T}\right)$. The quantities $N_{R L, R T}, N_{R L, F T}, N_{F L, R T}$, and $N_{F L, F T}$ can then be estimated from $N_{L L, L T}, N_{T L, L T}, N_{L L, T T}$ and $N_{T L, T T}$ by inverting a $4 \times 4$ matrix which is a function of the efficiencies for real and fake leptons to pass the tight requirements. The details of the Matrix Method are presented in Appendix B.

\section{Systematics}

Jet Systematics: There are three sources of uncertainty from jets: jet energy calibration, jet energy resolution, and jet reconstruction efficiency.

- Jet energy calibration is a rescaling of the momentum of jets in both data and Monte Carlo that is used to correct the energy of jets back to the particle level. The jet energy scale systematic is evaluated by varying it within the uncertainty.

- Jet energy resolution is an oversmearing of the jet momentum in Monte Carlo events to account for differences in the Monte Carlo and data jet resolutions. This systematic is evaluated by varying it within the uncertainty.

- The jet reconstruction efficiency is different between Monte Carlo and data. This effect is simulated by randomly removing Monte Carlo jets according to the scale factor (defined as the ratio of the data to Monte Carlo efficiencies). The scale factor is varied downward by $1 \sigma$ and then it is assumed that the 
uncertainty is symmetric about the central value. Only the downward variation is used because an upward variation gives a scale factor bigger than one in some cases, which can not then be simulated by the above procedure of randomly removing jets.

The uncertainties from jet systematics are fully correlated between the electron+track and muon+track channels and between the 1 and 2 jet bins.

Lepton Identification: Data to Monte Carlo scale factors must be applied to electrons, muons, and tracks in order to correct the efficiency for a Monte Carlo object to successfully pass all object identification cuts. Varying the electron scale factor within its uncertainty gives a systematic uncertainty on the electron+track cross section and likewise the muon scale factor produces a systematic in the muon+track channel. Scale factor uncertainties from electron and muon scale factors are uncorrelated between electron+track and muon+track channels but fully correlated between jet bins.

The track scale factors are a product of a track reconstruction scale factor, a quadratic $p_{T}$ parameterization and a quartic $\eta$ parameterization. There are four sets of parameters (all combinations of loose/tight and electron track/muon track scale factors). Each of these parameters is varied individually by $\pm 1 \sigma$ and the resulting uncertainty is calculated on an event-by-event basis. The track scale factor uncertainty is fully correlated between channels and jet bins. Track scale factors are discussed in Appendix A.

Opposite charge selection: The identified lepton and the isolated track are required to have opposite charge. The charge measurement is estimated to cause a $2 \%$ downward uncertainty [65]. Although muon tracks are not expected to have as large an uncertainty as electron tracks, here we conservatively apply a $2 \%$ uncertainty to both the electron+track and muon+track channels. This uncertainty is fully correlated between channels and jet multiplicity bins. 
Data Quality: The systematic uncertainty on the measurement of the data quality efficiency is estimated to be $0.5 \%$ [67]. This uncertainty is taken to be fully correlated between channels and jet multiplicity bins.

Vertex Identification: The estimated uncertainty from the primary vertex identification is $3 \%$ [68]. Additionally, there is an estimated $2.2 \%$ uncertainty due to differences between data and Monte Carlo in the z vertex position [69]. Vertex systematics are fully correlated between channels and jet mulitplicities.

Trigger Efficiencies: Monte Carlo events are weighted by the trigger efficiencies in order to reproduce the trigger selection as discussed in Sections 5.1 and 5.2. These efficiencies are $p_{T}$ and $\eta$ dependent. The systematic uncertainties from the trigger is estimated by varying the trigger efficiencies by $\pm 1 \sigma$. The trigger efficiency systematic is uncorrelated between the electron+track and muon+track channels but fully correlated between the 1 and 2 jet bins.

Normalization of Backgrounds: The normalization of the Z background is determined by normalizing the Monte Carlo to the data in a Z-dominated, low $\#_{T}$ sample as discussed in Section 6.4. Statistical uncertainties in the samples will give a systematic uncertainty on the cross section. We have also used the difference between the $\mathrm{K}$ factors calculated in the electron+track and muon+track channels as an additional systematic.

The normalizations of the fake backgrounds ( $\mathrm{W}+$ fakes and pure fakes) are determined by the Matrix Method. Statistical uncertainties on the samples $N_{L L, L T}$, $N_{T L, L T}, N_{L L, T T}, N_{T L, T T}$ gives a systematic uncertainty on the cross section. A second source of systematic uncertainty comes from the uncertainties on the measurement of the signal and background efficiencies. These uncertainties are fully uncorrelated between channels and jet multiplicities.

b Jet Identification: The systematic uncertainty due to b quark jet identification is evaluated by fluctuating within uncertainties the efficiencies for 
Monte Carlo jets to pass the b jet identification cut. This efficiency includes both the contribution from taggability and the tag rate functions (Section 5.4). b jet identification systematics are fully correlated between channels and jet multiplicities.

Luminosity: The uncertainty in the total integrated luminosity at DØ causes a systematic uncertainty in the cross section measurement of $6.1 \%$ [70].

Luminosity Profile: Monte Carlo events used in this analysis are generated with an overlay of zero bias events from data. The luminosity distribution of the zero bias events used for Monte Carlo generation does not precisely match the actual luminosity distribution in data. To estimate the size of the effect from this, the Monte Carlo was reweighted in order to match the Monte Carlo luminosity profile to the data profile, and the difference between this reweighted profile and the unweighted Monte Carlo was used as an additional systematic. Luminosity reweighting is only used for this systematic estimation.

\section{Results}

The purpose of the lepton+track analysis is to increase the sensitivity of the combined dilepton cross section. The lepton+track analysis will be presented in Section 8.1 and the effect of its inclusion in the combined dilepton cross section measurement is given in Section 8.2.

\subsection{Lepton + Track Cross Section}

The number of predicted and observed events used for the cross section measurement are shown in Table 12. The data and Monte Carlo distributions for various kinematic variables are shown in Appendix D.

In order to maximize the sensitivity of the analysis and minimize the effects of systematic uncertainties, each channel (electron+track and muon+track) and 


\begin{tabular}{|l|cc|cc|cc|}
\hline Source & \multicolumn{2}{|c|}{$\mathrm{e}+$ track } & \multicolumn{2}{|c|}{$\mu+$ track } & \multicolumn{2}{c|}{ combined } \\
& $\sigma^{-}(\mathrm{pb})$ & $\sigma^{+}(\mathrm{pb})$ & $\sigma^{-}(\mathrm{pb})$ & $\sigma^{+}(\mathrm{pb})$ & $\sigma^{-}(\mathrm{pb})$ & $\sigma^{+}(\mathrm{pb})$ \\
\hline Electron ID & -0.25 & 0.28 & & & -0.16 & 0.16 \\
Muon ID & & & -0.04 & 0.04 & -0.01 & 0.02 \\
Muon Track Quality & & & -0.04 & 0.04 & -0.01 & 0.02 \\
Muon Isolation & & & -0.11 & 0.12 & -0.05 & 0.05 \\
Opp. Charge Sel. & 0.00 & 0.10 & 0.00 & 0.11 & 0.00 & 0.10 \\
Vertex ID & 0.00 & 0.15 & 0.00 & 0.16 & 0.00 & 0.16 \\
Vertex Z Simulation & -0.10 & 0.11 & -0.11 & 0.12 & -0.11 & 0.11 \\
Data Quality & -0.02 & 0.02 & -0.03 & 0.03 & -0.02 & 0.02 \\
Jet Scale Calib. & -0.13 & 0.10 & -0.10 & 0.17 & -0.12 & 0.13 \\
Jet Resolution & -0.02 & 0.00 & 0.00 & 0.00 & -0.02 & 0.00 \\
Jet Reco. Eff. & -0.02 & 0.02 & -0.01 & 0.01 & -0.02 & 0.02 \\
TRFs / Taggability & -0.24 & 0.27 & -0.28 & 0.32 & -0.26 & 0.29 \\
Track ID & -0.51 & 0.57 & -0.71 & 0.80 & -0.60 & 0.67 \\
Trigger (e+jets) & -0.14 & 0.18 & & & -0.08 & 0.11 \\
Trigger ( $\mu+j$ jets) & & & -0.40 & 0.58 & -0.17 & 0.23 \\
MC Statistics & -0.07 & 0.07 & -0.10 & 0.10 & -0.06 & 0.06 \\
K Factors & -0.24 & 0.31 & -0.28 & 0.39 & -0.18 & 0.30 \\
Luminosity Profile & -0.20 & 0.20 & -0.02 & 0.02 & -0.11 & 0.11 \\
Mat. Meth. Effs. & -0.14 & 0.28 & -0.18 & 0.29 & -0.13 & 0.26 \\
Mat. Meth. Statistics & -0.10 & 0.10 & -0.12 & 0.12 & -0.07 & 0.08 \\
\hline Total & -0.75 & 0.89 & -0.96 & 1.20 & -0.77 & 0.93 \\
\hline
\end{tabular}

Table 11: Table of systematic uncertainties in the electron+track, muon+track, and combined channels.

\begin{tabular}{lrrrr} 
Sample & e+track, 1 jet & e+track, 2 jets & $\mu$ +track, 1 jet & $\mu$ +track, 2 jets \\
\hline $\mathrm{t} \overline{\mathrm{t}}$ & 1.37 & 7.23 & 0.94 & 5.71 \\
$\mathrm{Z} \rightarrow \mathrm{ll}$ & 0.90 & 0.85 & 0.95 & 1.18 \\
$\mathrm{Z} \rightarrow \tau \tau$ & 0.21 & 0.17 & 0.19 & 0.19 \\
$\mathrm{WW}$ & 0.05 & 0.03 & 0.04 & 0.03 \\
$\mathrm{WZ}$ & 0.01 & 0.02 & 0.01 & 0.02 \\
$\mathrm{ZZ}$ & 0.02 & 0.06 & 0.02 & 0.04 \\
$\mathrm{~W}+$ fake & 0.14 & 0.48 & 0.10 & -0.22 \\
QCD & 0.21 & 0.21 & 0.03 & 0.00 \\
\hline Total Predicted & 2.97 & 9.10 & 2.34 & 7.04 \\
\hline Observed & 4 & 8 & 1 & 8
\end{tabular}

Table 12: Number of predicted and observed events. t $\overline{\mathrm{t}}$ estimate based on a $5.0 \mathrm{pb}$ cross section. 
both jet multiplicity bins are used in the cross section analysis, resulting in four independent channels. The cross section is determined by maximizing the product of the likelihoods. The number of expected events in channel $i$ is:

$$
\tilde{N}_{i}=\sigma B R \mathcal{L} \epsilon_{i}+N_{i}^{b k g}
$$

where $\sigma$ is the $t \overline{\mathrm{t}}$ cross section, $B R$ is the branching fraction of $t \overline{\mathrm{t}}$ events to dileptons, $\mathcal{L}$ is the luminosity, $\epsilon_{i}$ is the efficiency for t $\overline{\mathrm{t}}$ events to pass the selection cuts and $N_{i}^{b k g}$ is the number of expected background events. The likelihood is then the product of the Poisson distributions:

$$
\mathscr{L}\left(\sigma,\left[N_{i}^{o b s} N_{i}^{b k g}, B R, \mathcal{L}, \epsilon_{i}\right]\right)=\prod_{i=1}^{4} \frac{\tilde{N}_{i}^{N_{i}^{o b s}}}{N_{i}^{\text {obs }}} e^{-\tilde{N}_{i}}
$$

\begin{tabular}{|l|rrr|}
\hline Channel & Branching Ratio & Luminosity & $\epsilon$ \\
\hline e+track, 1 jet & 0.1066 & $1036 \mathrm{pb}^{-1}$ & $0.25 \%$ \\
e+track, 2 jet & 0.1066 & $1036 \mathrm{pb}^{-1}$ & $1.31 \%$ \\
$\mu+$ track, 1 jet & 0.1066 & $994 \mathrm{pb}^{-1}$ & $0.18 \%$ \\
$\mu+$ track, 2 jet & 0.1066 & $994 \mathrm{pb}^{-1}$ & $1.08 \%$ \\
\hline
\end{tabular}

Table 13: Inputs to the cross section calculation.

The cross sections for the individual and combined channels, derived with a tĒ Monte Carlo set with top mass $175 \mathrm{GeV}$, are:

$$
\begin{array}{cc}
\mathrm{e}+\text { track }: & \sigma=4.7_{-1.8}^{+2.2}(\text { stat })_{-0.8}^{+0.9} \text { (syst) } \pm 0.3 \text { (lumi) } \mathrm{pb} \\
\mu+\text { track }: & \sigma=5.3_{-2.0}^{+2.5}(\text { stat })_{-1.0}^{+1.2}(\text { syst }) \pm 0.3(\text { lumi }) \mathrm{pb} \\
\text { combined : } & \sigma=5.0_{-1.4}^{+1.6}(\text { stat })_{-0.8}^{+0.9}(\text { syst }) \pm 0.3 \text { (lumi) pb }
\end{array}
$$

The cross section measurement is dependent on the presumed mass of the top quark. The cross section has been evaluated with t $\bar{t}$ Monte Carlo with a mass 


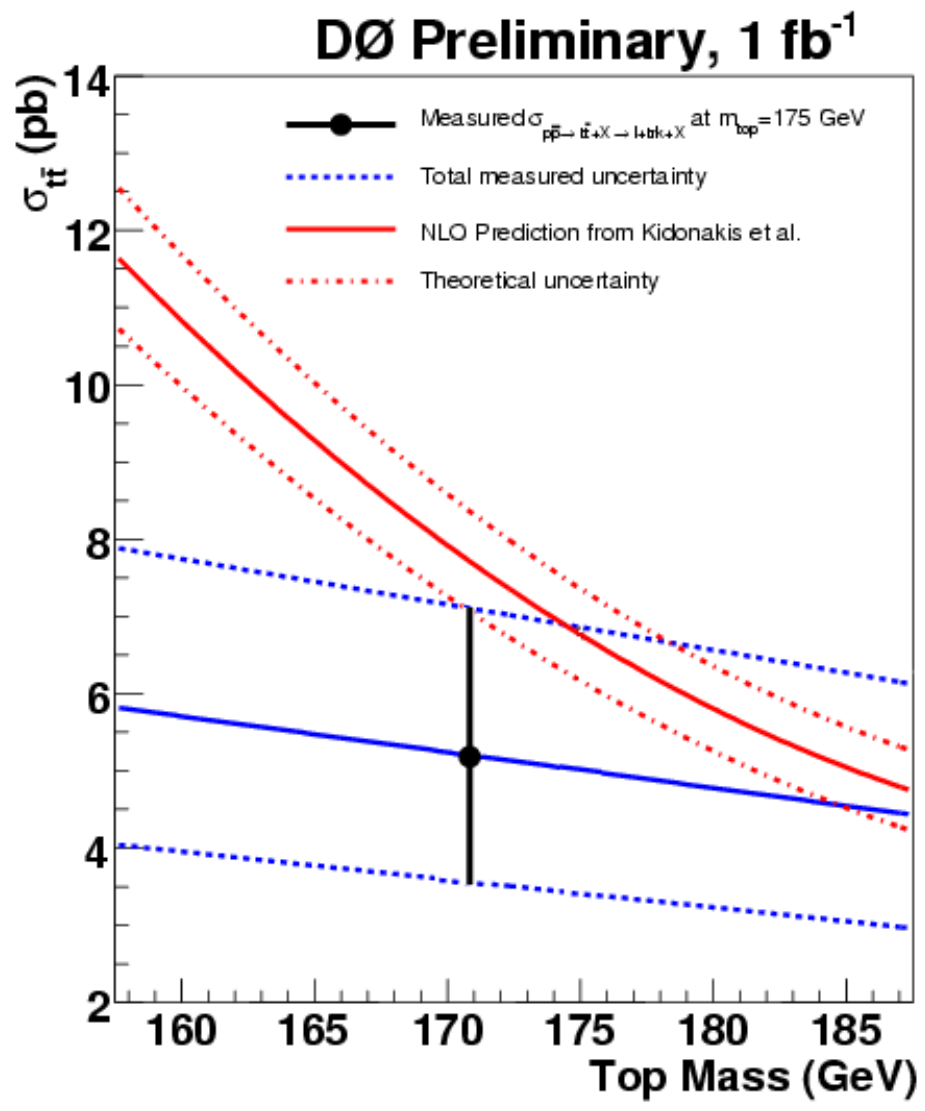

Figure 25: Measured cross section vs. assumed mass of the top quark.

of 165,175 , and $185 \mathrm{GeV}$. A linear fit of these three points gives a mass dependence (in units of $\mathrm{pb}$ ) of the form

$$
\sigma\left(M_{t o p}\right)=13.13-0.04644 M_{t o p}
$$

Evaluating this at the top quark world average mass of $170.9 \mathrm{GeV}$ gives

$$
\sigma\left(\mathrm{M}_{\mathrm{top}}=170.9 \mathrm{GeV}\right)=5.2_{-1.4}^{+1.6}(\text { stat })_{-0.8}^{+0.9} \text { (syst) } \pm 0.3 \text { (lumi) } \mathrm{pb}
$$

The cross section as a function of top mass is shown in Figure 25. 


\subsection{Combined Dilepton Cross Section}

The calculation for the combined dilepton cross section [1] is very similar to the lepton+track discussion in Section 8.1 except that now, in addition to the four channels in lepton+track (electron or muon channels with one or two jets), there are four additional channels: electron-electron with 2 or more jets, muon-muon with 2 or more jets, electron-muon with 1 jet, and electron-muon with 2 or more jets (electron-electron and muon-muon were not measured in the 1 jet channel). The likelihood function is identical to equation (34), except that now the index will run from one to eight:

$$
\begin{aligned}
\mathscr{L}\left(\sigma,\left[N_{i}^{\text {obs }} N_{i}^{b k g}, B R, \mathcal{L}, \epsilon_{i}\right]\right) & =\prod_{i=1}^{8} \frac{\tilde{N}_{i}^{N_{i}^{o b s}}}{N_{i}^{o b s} !} e^{-\tilde{N}_{i}} \\
\tilde{N}_{i} & =\sigma B R \mathcal{L} \epsilon_{i}+N_{i}^{b k g}
\end{aligned}
$$

The relevant data needed to compute the likelihood function is presented in Tables 14 and 15 for all eight channels. In Table 15 the branching ratio listed is the branching ratio for $t \bar{t}$ pairs decaying into the given final state - electron-electron, electron-muon, or muon-muon (and the possibility of the original $\mathrm{W}$ decaying to a tau which decays to an electron or muon is included). However, in Table 14, the identity of the lepton corresponding to the track is not known, so the listed branching ratio is the branching ratio of $t \bar{t}$ pairs to all possible dilepton final states. Similarly, the selection efficiency $\epsilon$ in these tables is measured with respect to either

the given final state (Table 15) or with respect to all dilepton final states (Table 14). The measured cross sections for the fully identified lepton channels [1] are: 


$$
\begin{array}{cc}
\mathrm{e}+\mathrm{e}: & \sigma=9.6_{-2.7}^{+3.2}(\text { stat })_{-1.6}^{+1.9}(\text { syst }) \pm 0.6(\text { lumi }) \mathrm{pb} \\
\mathrm{e}+\mu: & \sigma=6.1_{-1.2}^{+1.4}(\text { stat })_{-0.7}^{+0.8}(\text { syst }) \pm 0.4(\text { lumi }) \mathrm{pb} \\
\mu+\mu: & \sigma=6.5_{-3.2}^{+4.0}(\text { stat })_{-0.9}^{+1.1}(\text { syst }) \pm 0.4(\text { lumi }) \mathrm{pb}
\end{array}
$$

\begin{tabular}{|l|rrr|r|r|}
\hline Channel & $N^{\text {obs }}$ & $N^{b k g}$ & BR & Lumi & $\epsilon$ \\
\hline e+track, 1 jet & 4 & 1.58 & 0.1066 & $1036 \mathrm{pb}^{-1}$ & $0.25 \%$ \\
e+track, 2+ jets & 8 & 1.83 & 0.1066 & $1036 \mathrm{pb}^{-1}$ & $1.31 \%$ \\
$\mu+$ track, 1 jet & 1 & 1.38 & 0.1066 & $994 \mathrm{pb}^{-1}$ & $0.18 \%$ \\
$\mu+$ track, $2^{+}$jets & 8 & 1.36 & 0.1066 & $994 \mathrm{pb}^{-1}$ & $1.08 \%$ \\
\hline
\end{tabular}

Table 14: Inputs to the cross section calculation from the lepton+track channels. The listed branching ratio and efficiency is given relative to $t \bar{t}$ decays to all dilepton final states ee, $\mathrm{e} \mu, \mathrm{e} \tau, \mu \mu, \mu \tau$, and $\tau \tau$.

\begin{tabular}{|l|rrr|r|r|}
\hline Channel & $N^{\text {obs }}$ & $N^{b k g}$ & BR & Lumi & $\epsilon$ \\
\hline ee, $2^{+}$jets & 16 & 3.0 & 0.01584 & $1036 \mathrm{pb}^{-1}$ & $8.3 \%$ \\
$\mathrm{e} \mu, 1$ jet & 16 & 10.2 & 0.03155 & $1046 \mathrm{pb}^{-1}$ & $3.1 \%$ \\
$\mathrm{e} \mu, 2^{+}$jets & 32 & 6.7 & 0.03155 & $1046 \mathrm{pb}^{-1}$ & $12.4 \%$ \\
$\mu \mu, 2^{+}$jets & 9 & 3.6 & 0.01571 & $1046 \mathrm{pb}^{-1}$ & $5.1 \%$ \\
\hline
\end{tabular}

Table 15: Inputs to the cross section calculation from the fully identified dilepton channels. The given branching fraction and efficiency is relative to t $\overline{\mathrm{t}}$ decay to the listed final state $(\mathrm{ee}, \mathrm{e} \mu, \mu \mu)$, either by direct decay of the $\mathrm{W}(W \rightarrow l \nu)$ or through a $\tau$ intermediary $(W \rightarrow \tau \nu \rightarrow l \nu \nu)$.

All of the Monte Carlo samples used for the various dilepton channels used a top mass of $175 \mathrm{GeV}$. The cross section calculated at this mass is:

$$
\sigma=6.2_{-0.9}^{+0.9}(\text { stat })_{-0.7}^{+0.8} \text { (syst) } \pm 0.4 \text { (lumi) pb }
$$

The final cross section, calculated at the world combined average top mass of $170.9 \mathrm{GeV}$ is 


$$
\sigma\left(\mathrm{M}_{\text {top }}=170.9 \mathrm{GeV}\right)=6.4_{-0.9}^{+0.9}(\text { stat })_{-0.7}^{+0.8}(\text { syst }) \pm 0.4 \text { (lumi) } \mathrm{pb}
$$

\subsection{Conclusion and Outlook}

The cross sections for the individual channels, the dilepton combination, and the lepton+jets channel are shown in Figure 26 for an assumed top mass of $175 \mathrm{GeV}$. The lepton+jets channel, where one top quark decays leptonically and the other decays into quarks, is the most precisely measured channel for the top quark cross

section [75]. The individual dilepton channels are in agreement with each other to around the $1 \sigma$ level, and the dilepton and lepton+jets channel measurements agree with each other. Furthermore, these measurements are all consistent with Standard Model predictions.

Previous dilepton studies have typically had statistical uncertainties much larger than the systematic uncertainties. However, with the inclusion of new channels such as the lepton+track channel as well as substantial luminosity upgrades to the Tevatron, the statistical uncertainty has now been reduced down to very nearly the same size as the systematics. Further detector studies may be required in the future to reduce systematic uncertainties in order to take full advantage of the new statistics available. 


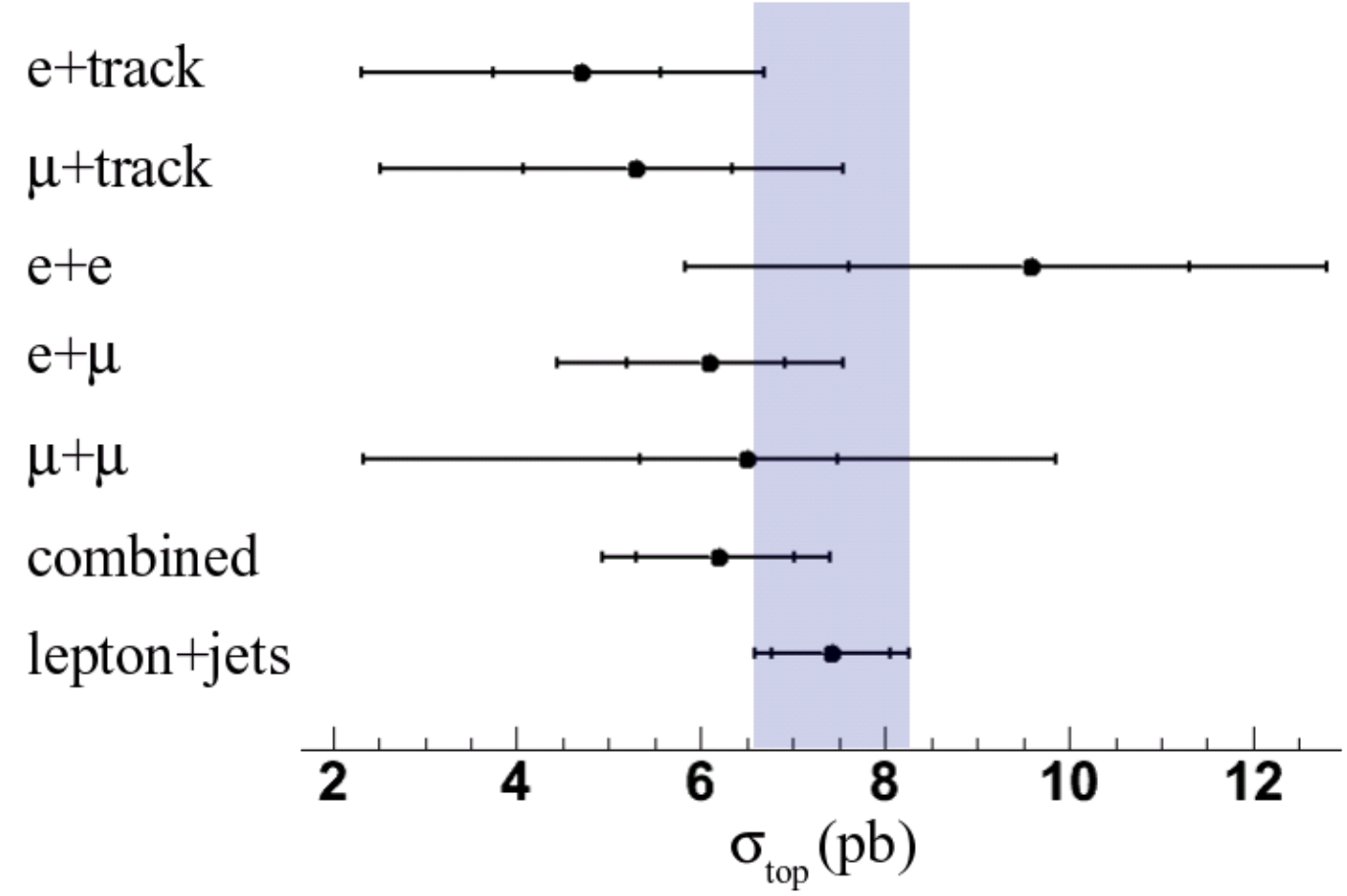

Figure 26: Cross sections for the individual dilepton channels, the dilepton combination, and the lepton+jets channel for an assumed top mass of $175 \mathrm{GeV}$. The inner error bars are the systematic error (including the luminosity systematic), and the outer error bars are the total error (statistical plus systematic). 


\section{A Track Identification Scale Factors}

It has been found that the Monte Carlo detector simulation does not correctly reproduce the track reconstruction efficiency found in data. We therefore choose to derive a reweighting factor which will be applied to Monte Carlo events. This scale factor is defined as the data reconstruction efficiency divided by the Monte Carlo reconstruction efficiency, and is calculated with $\mathrm{Z} \rightarrow$ ee and $\mathrm{Z} \rightarrow \mu \mu$ events using a tag-and-probe method. Events which are unbiased with respect to the track identification cuts must be selected. Due to effects such as bremsstralung with the tracking system materials, electron tracks have a substantially different behavior than muon tracks. Therefore, a separate scale factor is derived for electron tracks

and muon tracks. In the final analysis the electron track scale factor is applied to tracks that are matched to Monte Carlo electrons; otherwise, the muon track scale factor is applied.

The scale factor for electron tracks is calculated from $Z \rightarrow$ ee events. Events are selected which have two high $p_{T}$ electrons with a combined mass between 80 $\mathrm{GeV}$ and $100 \mathrm{GeV}$. Very tight selection cuts are placed on the electrons, and they are required to be track matched. One electron is randomly assigned to be the tag electron, and the other electron is the probe which will be used to determine the efficiencies. The tag electron is required to pass the electron trigger used in this study, and the tag's track must pass all tight track identification cuts discussed in Section 4.3.

The muon track scale factor is determined from $\mathrm{Z} \rightarrow \mu \mu$ events, which must contain two high $p_{T}$ muons with a mass between 80 and $100 \mathrm{GeV}$. Both muons must pass extremely tight selection requirements and be track matched. One muon is randomly assigned to be the tag muon, and the other muon is the probe which will be used to determine the efficiencies. Similar to the electron case, the tag muon 
must satisfy a muon trigger and the tag muon's track must meet the tight track selection from Section 4.3.

Monte Carlo events used to determine the efficiency in Monte Carlo are generated using the Alpgen event generator (see Section 6.2 for a discussion of Alpgen). For either the electron or muon case, the probe lepton is used to derive the efficiency, which is parameterized as a function of $p_{T}$ and $\eta$. The scale factor is parameterized as a quadratic function in $p_{T}$ and a quartic function in $\eta$, and it is assumed that there is no correlation between the $p_{T}$ and $\eta$ parameterizations:

$$
\text { Scale Factor }=\frac{1}{\frac{\epsilon_{D A T A}}{\epsilon_{M C}}}\left(a_{2} p_{T}^{2}+a_{1} p_{T}+a_{0}\right) \times\left(b_{4} \eta^{4}+b_{3} \eta^{3}+b_{2} \eta^{2}+b_{1} \eta+b_{0}\right)
$$

The factor of $\frac{1}{\frac{\epsilon_{D A T A}}{\epsilon_{M C}}}$, which is just the reciprocal of the overall efficiency correction, is necessary because the product of the quadratic term in $p_{T}$ and the quartic term in $\eta$ double counts the overall efficiency, so it must be divided out once.

The scale factors and their parameterizations are shown in Figures 27 - 30 for electron+track and Figures 31 - 34 for muon+track. In order to test the validity of this parameterization, a closure test is performed. In a closure test, the scale factors are applied to the same Monte Carlo events which were used to derive the scale factors. Performing the same scale-factor derivation on these reweighted events should yield a scale factor of nearly unity. The closure test is shown in Figures 35 36. 


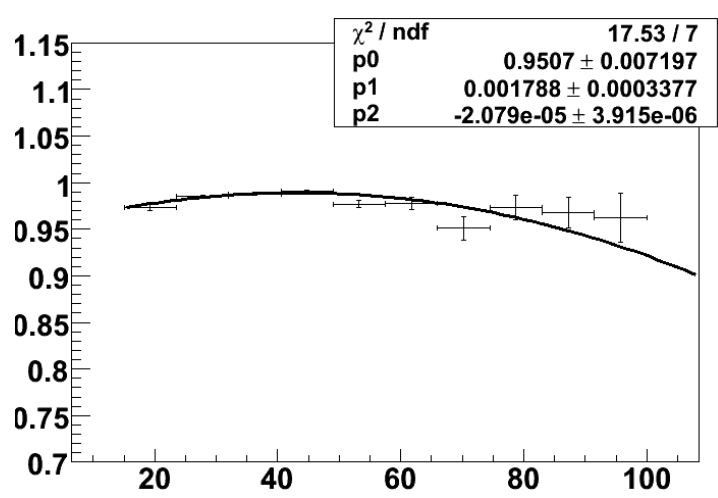

(a)

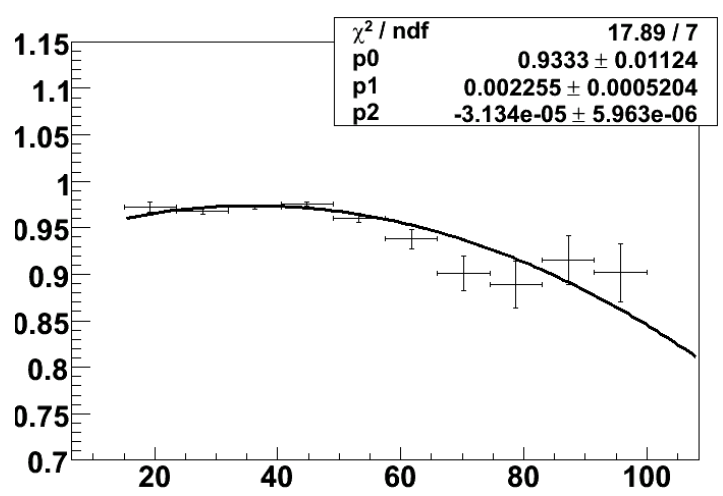

(c)

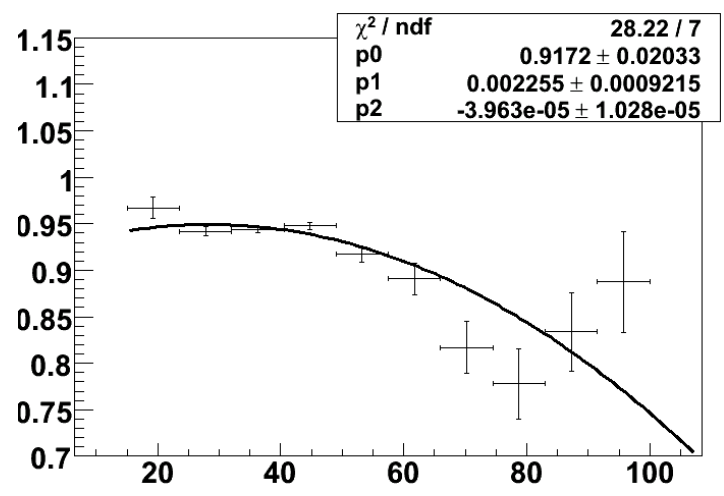

(e)

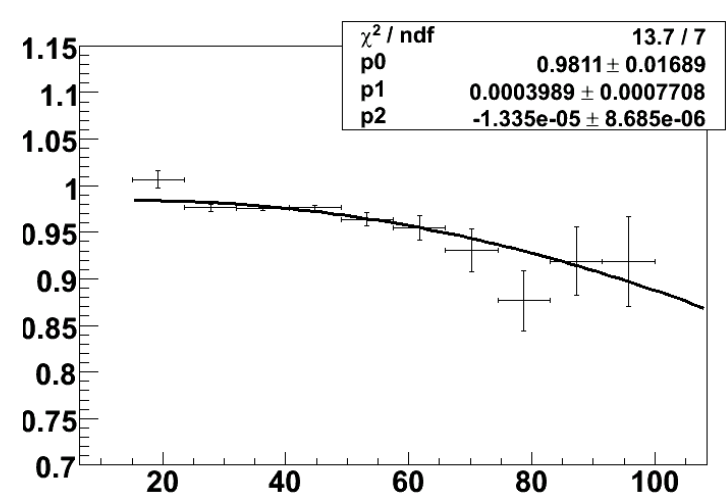

(b)

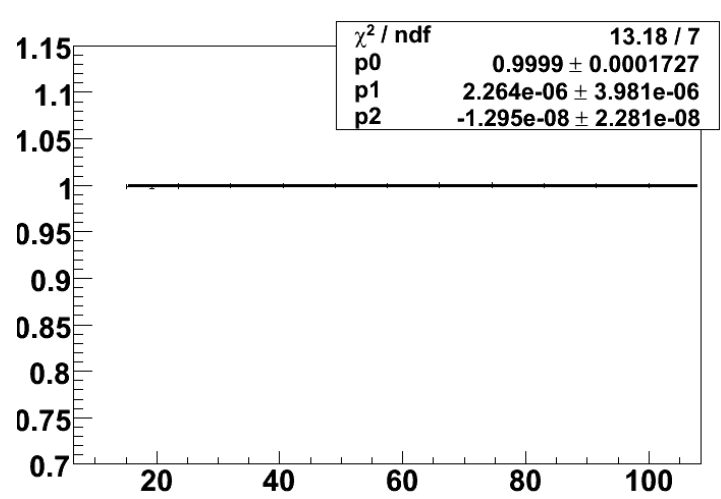

(d)

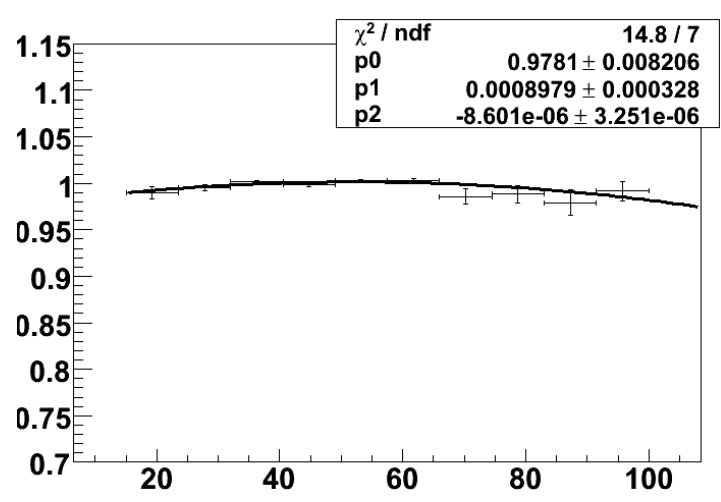

(f)

Figure 27: Monte Carlo to data scale factor of the track in the electron+track channel as a function of $p_{T}$ for (a) the $\Delta z$ (lepton vertex, track) cut, (b) the distance-fromvertex significance cut, (c) the $\chi^{2}$ cut, (d) the loose track isolation cut, (e) all loose cuts combined, and (f) the tight track isolation cut. 


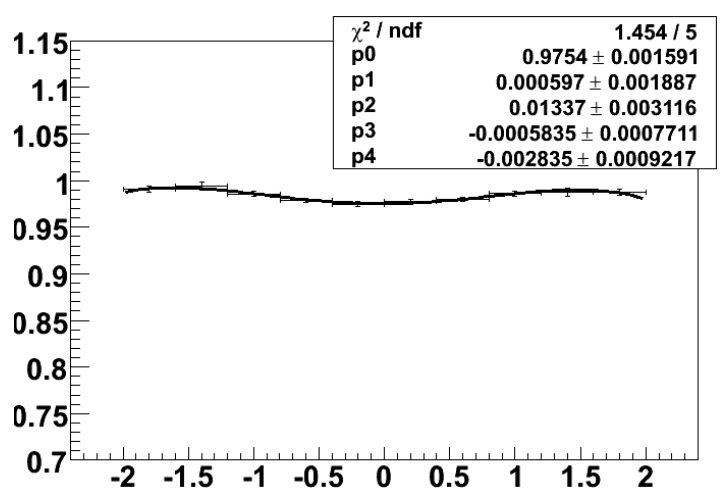

(a)

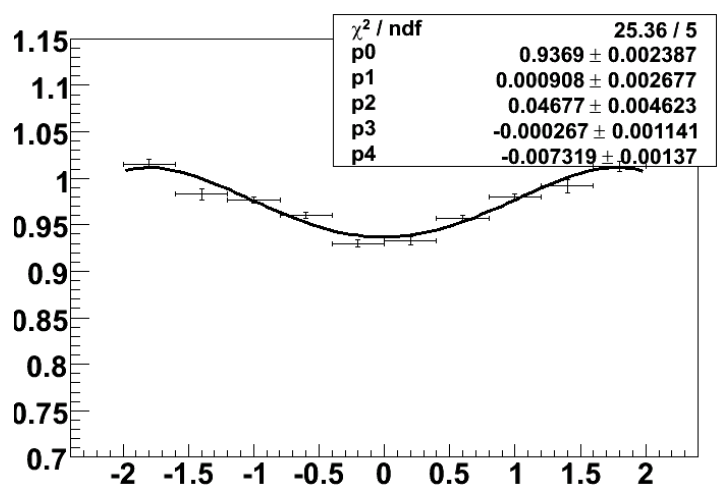

(c)

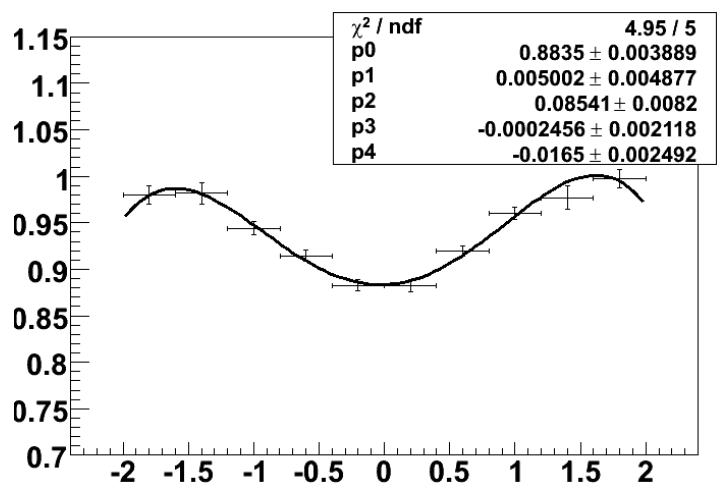

(e)

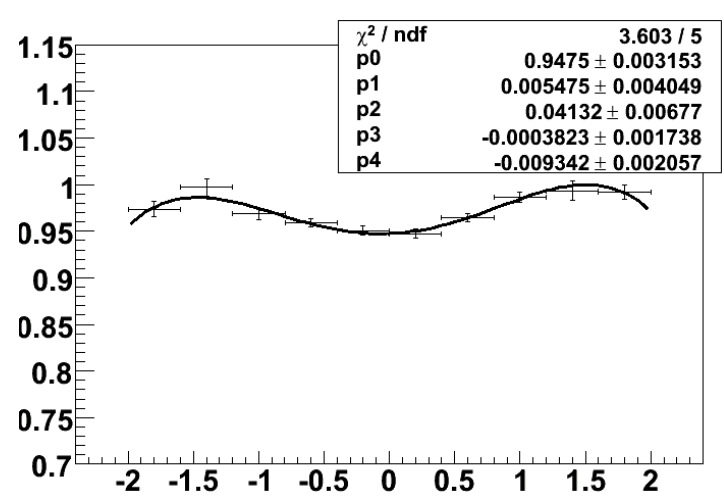

(b)

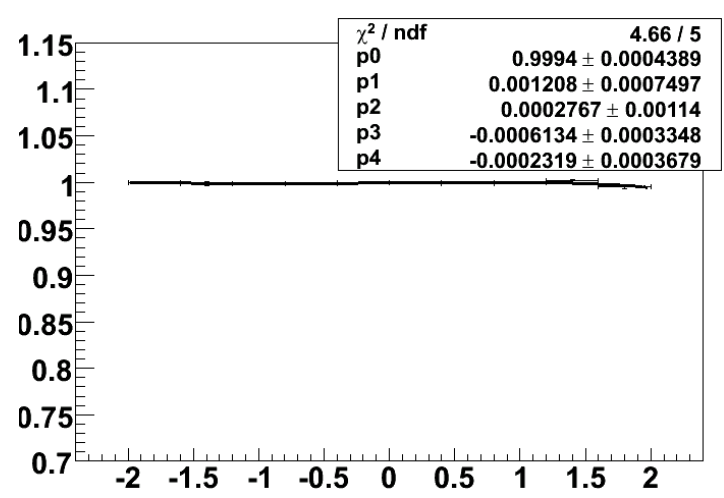

(d)

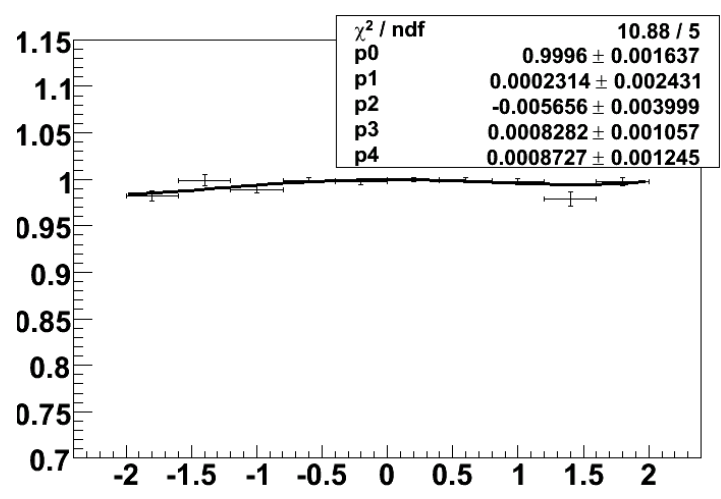

(f)

Figure 28: Monte Carlo to data scale factor of the track in the electron+track channel as a function of $\eta$ for (a) the $\Delta z$ (lepton vertex, track) cut, (b) the distance-fromvertex significance cut, (c) the $\chi^{2}$ cut, (d) the loose track isolation cut, (e) all loose cuts combined, and (f) the tight track isolation cut. 


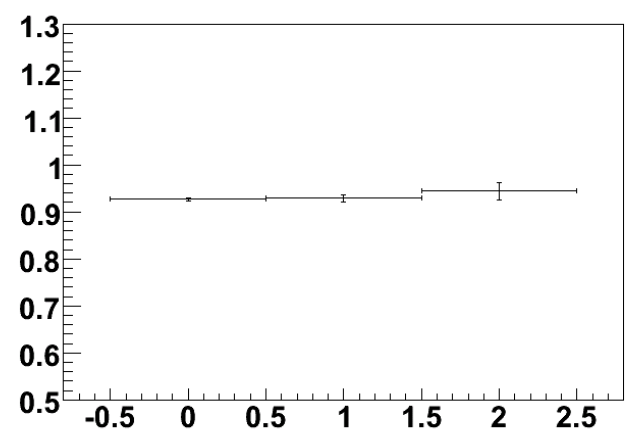

(a)

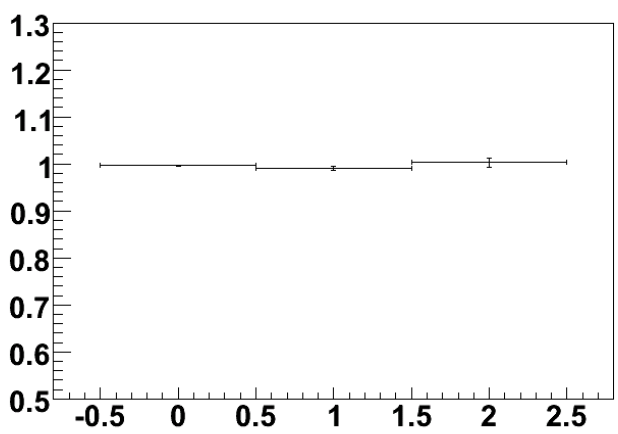

(b)

Figure 29: Monte Carlo to data scale factor of the track in the electron+track channel as a function of number of jets for (a) all loose cuts combined, and (b) the tight track isolation cut.

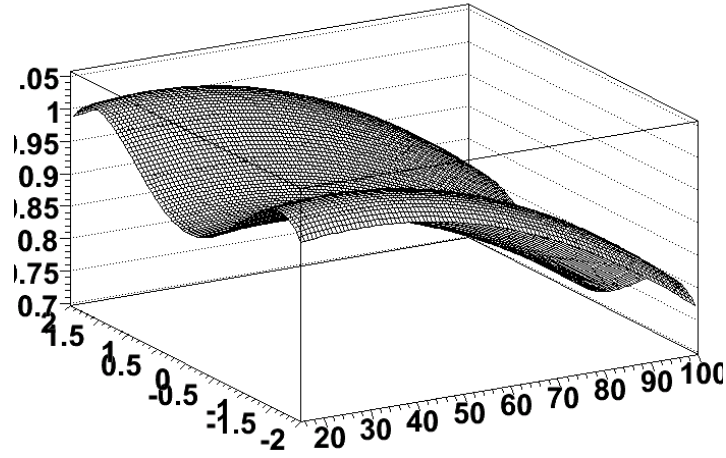

(a)

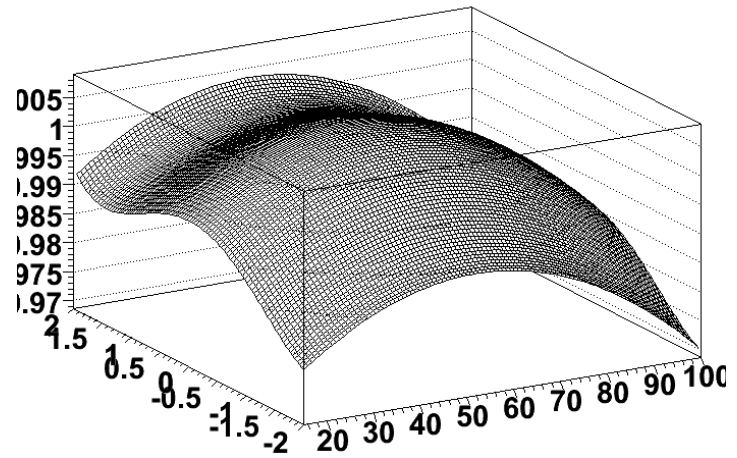

(b)

Figure 30: 2D representation of Monte Carlo to data scale factor in the electron+track channel for (a) all loose cuts combined, and (b) the tight track isolation cut. 


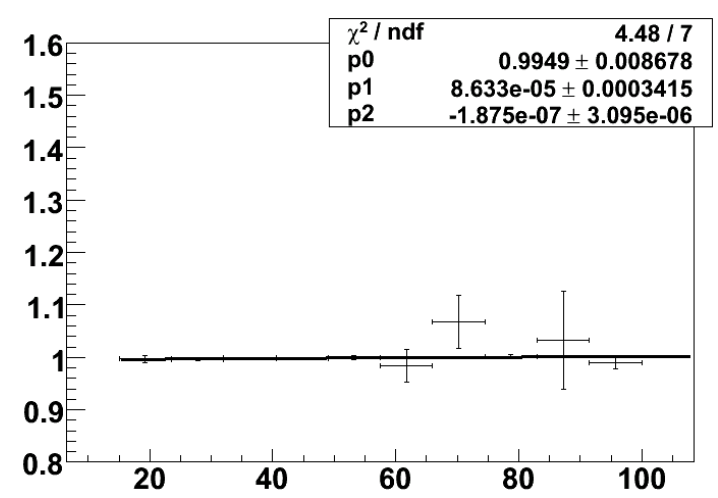

(a)

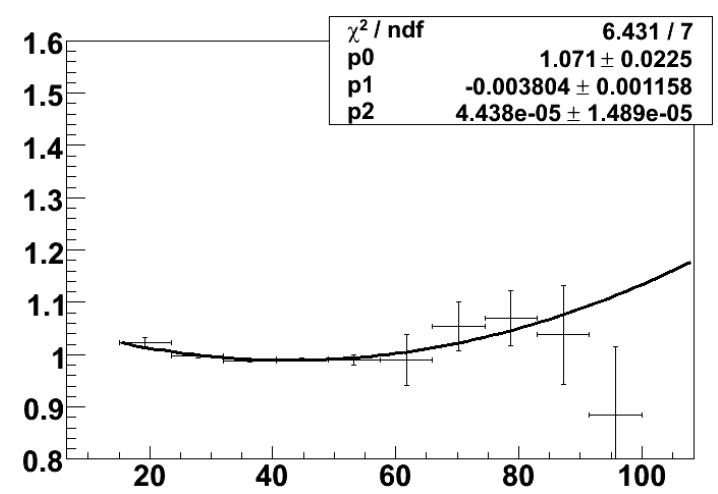

(c)

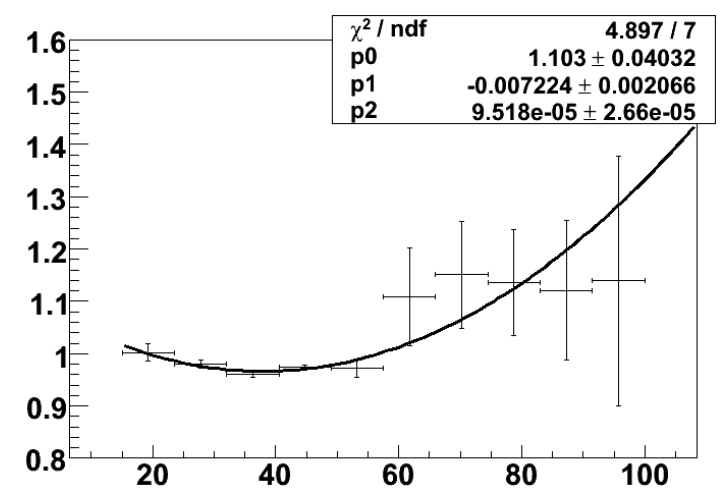

(e)

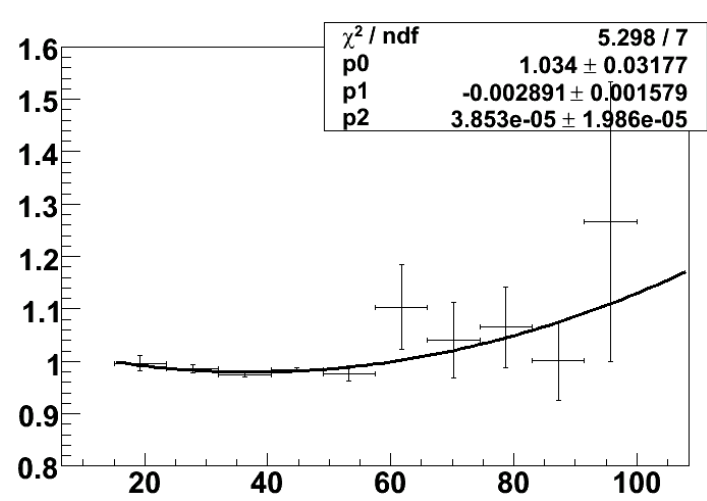

(b)

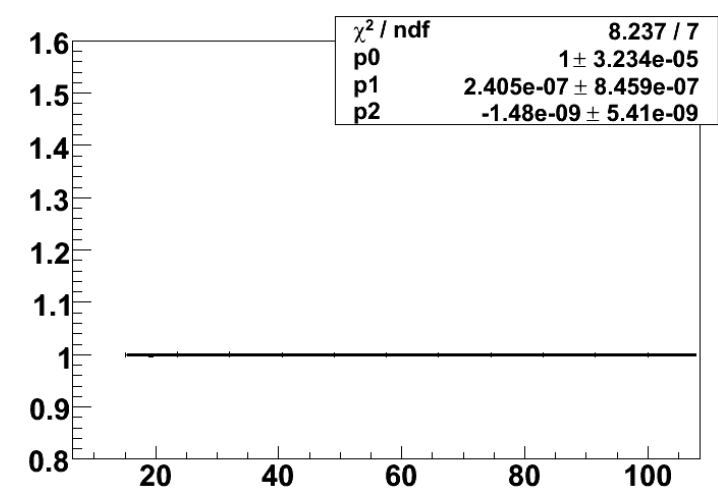

(d)

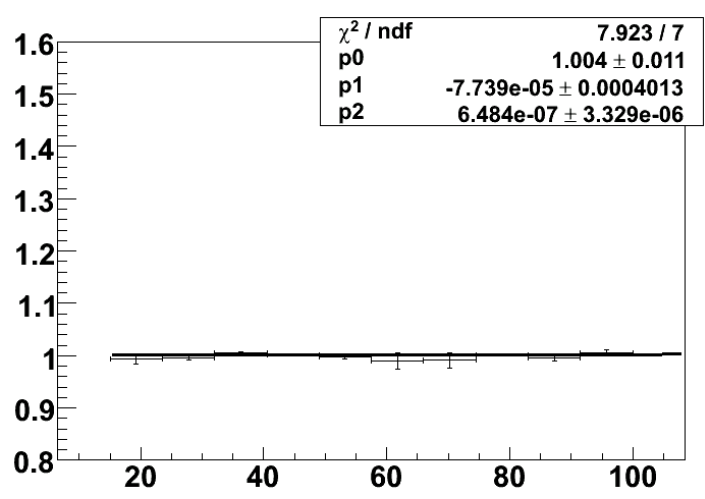

(f)

Figure 31: Monte Carlo to data scale factor of the track in the muon+track channel as a function of $p_{T}$ for (a) the $\Delta$ (lepton vertex, track) cut, (b) the distance-fromvertex significance cut, (c) the $\chi^{2}$ cut, (d) the loose track isolation cut, (e) all loose cuts combined, and (f) the tight track isolation cut. 


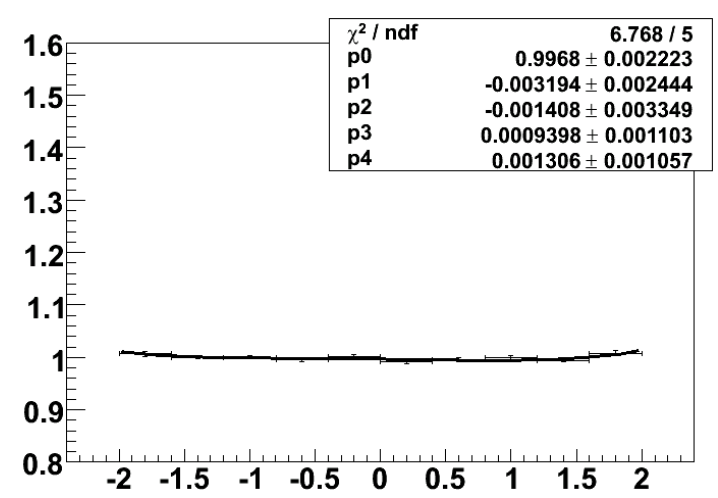

(a)

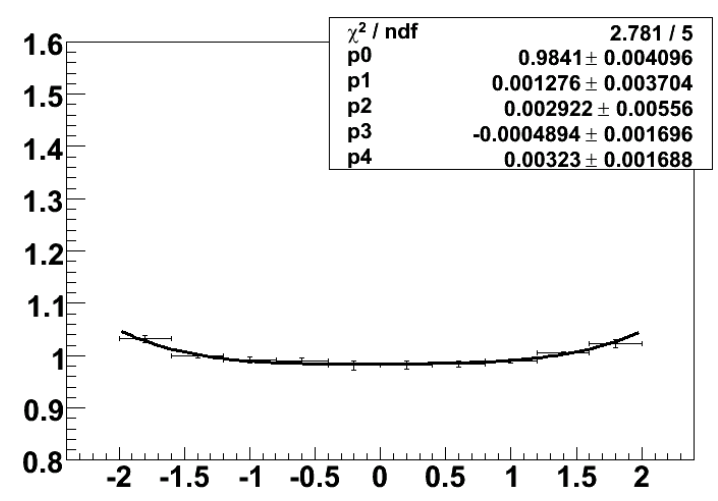

(c)

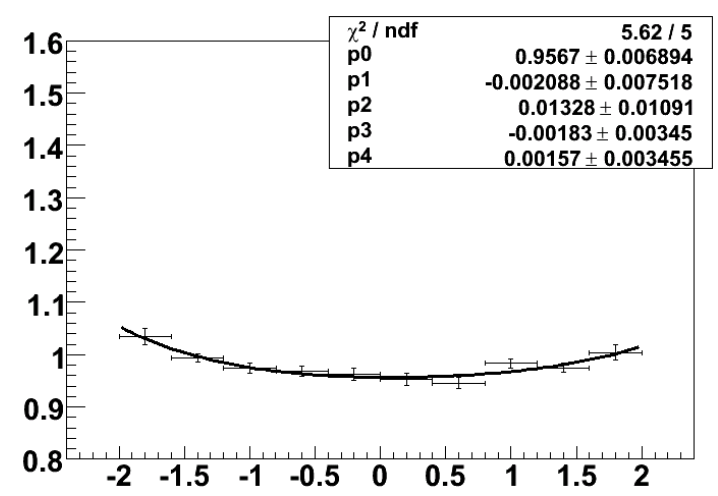

(e)

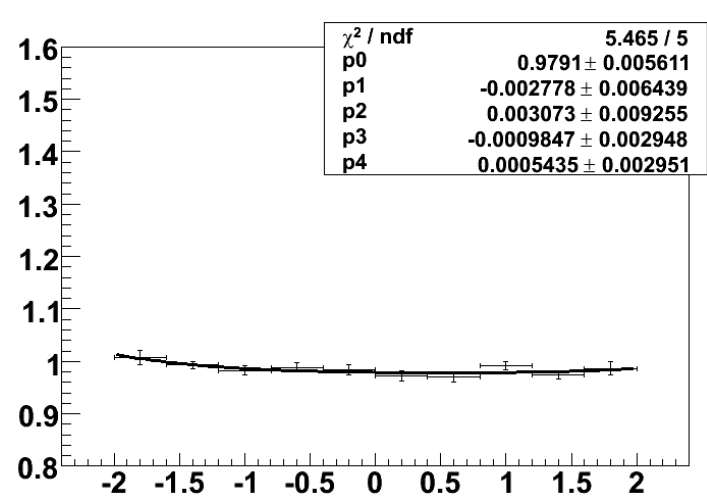

(b)

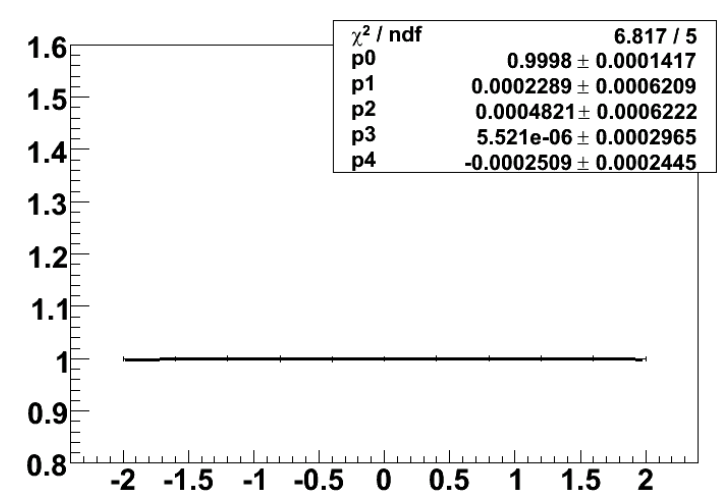

(d)

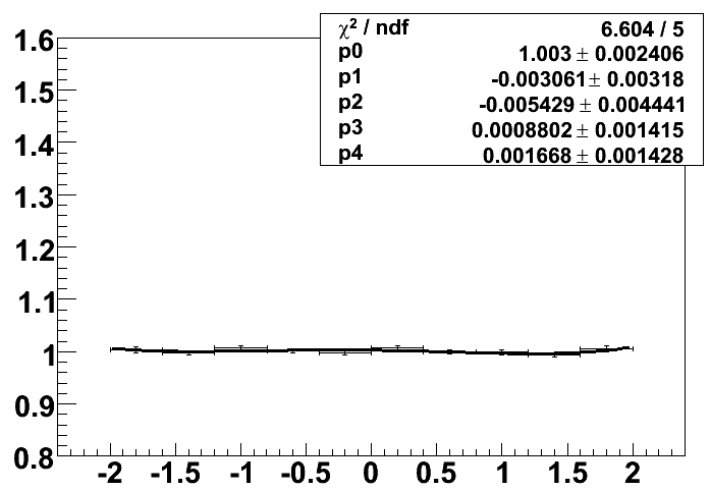

(f)

Figure 32: Monte Carlo to data scale factor of the track in the muon+track channel as a function of $\eta$ for (a) the $\Delta$ z(lepton vertex, track) cut, (b) the distance-from-vertex significance cut, (c) the $\chi^{2}$ cut, (d) the loose track isolation cut, (e) all loose cuts combined, and (f) the tight track isolation cut. 


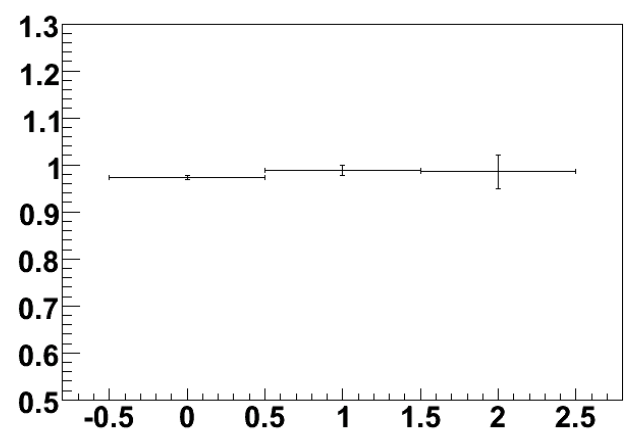

(a)

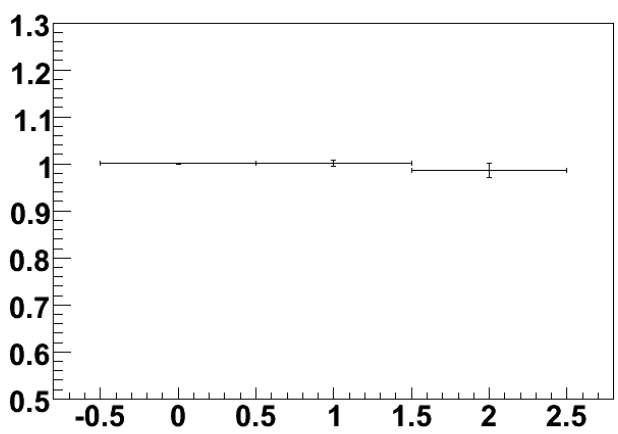

(b)

Figure 33: Monte Carlo to data scale factor of the track in the muon+track channel as a function of number of jets for (a) all loose cuts combined, and (b) the tight track isolation cut.

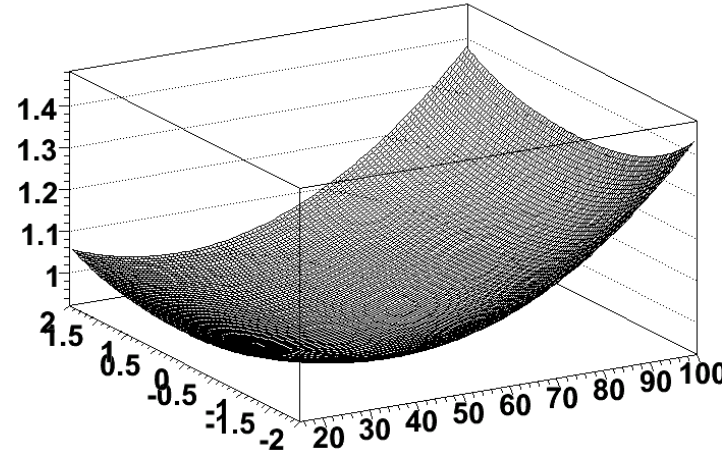

(a)

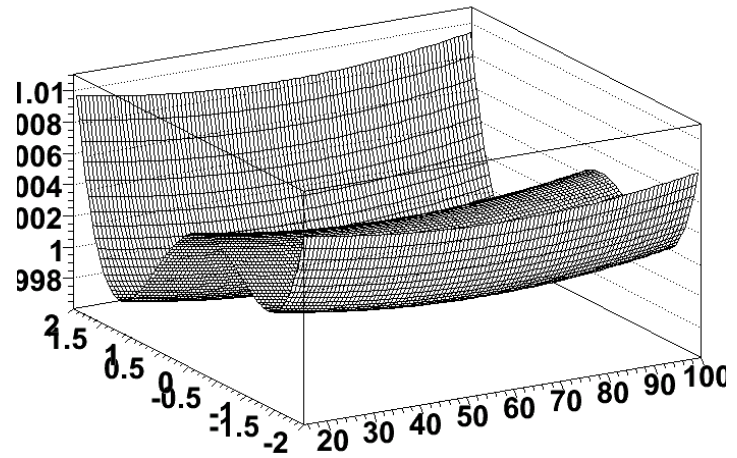

(b)

Figure 34: 2D representation of Monte Carlo to data scale factor in the muon+track channel for (a) all loose cuts combined, and (b) the tight track isolation cut. 


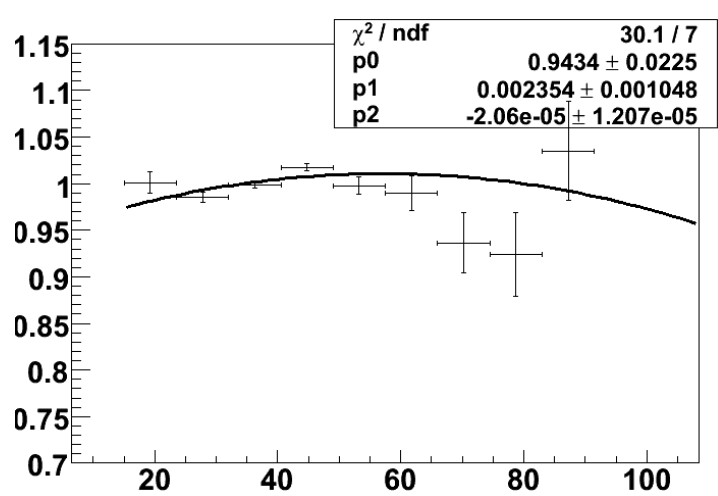

(a)

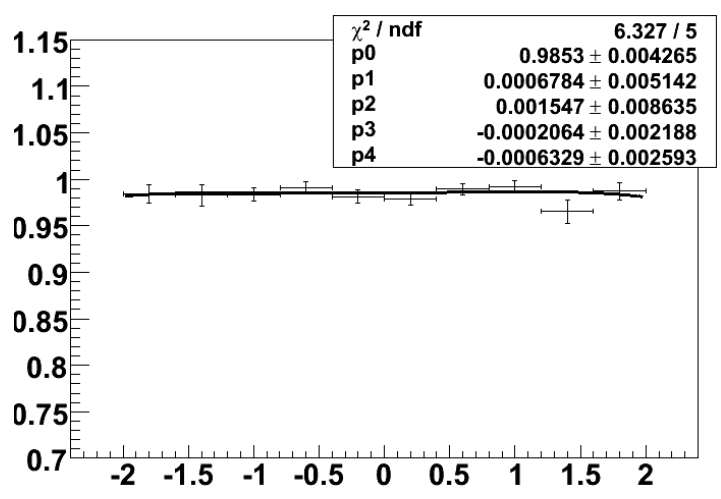

(c)

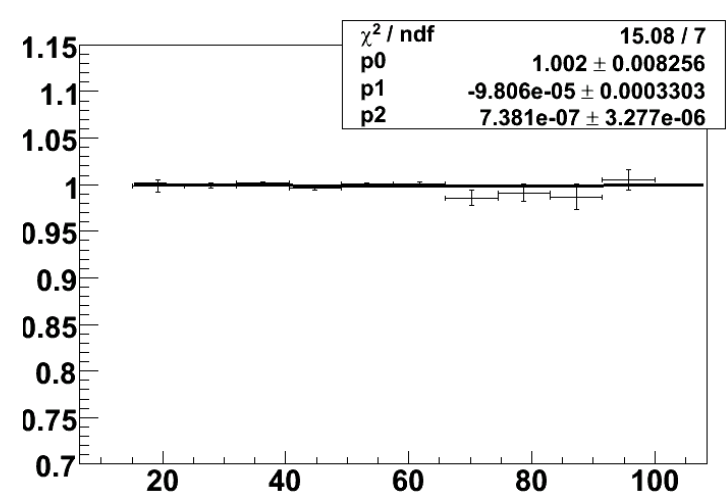

(b)

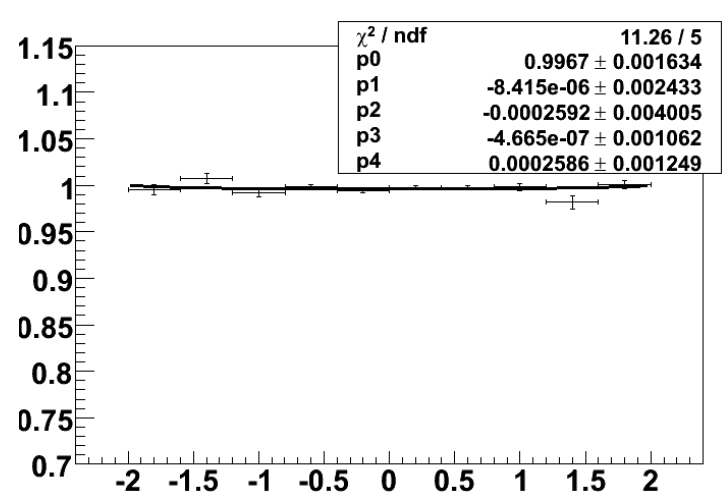

(d)

Figure 35: Closure test in the electron+track channel for (a) loose track scale factor as a function of $p_{T}$ (b) tight track scale factor as a function of $p_{T}$ (c) loose track scale factor as a function of $\eta$ (d) tight scale track factor as a function of $\eta$. 


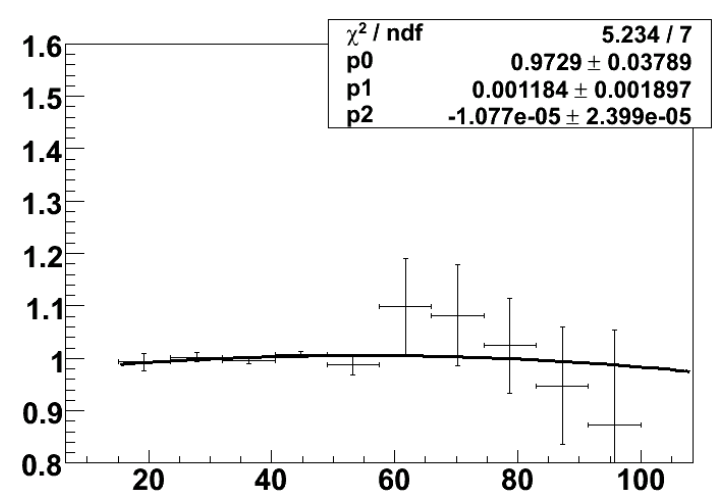

(a)

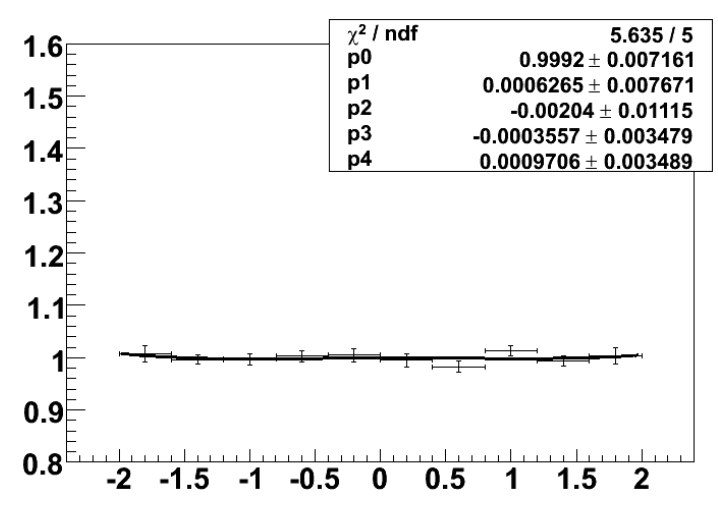

(c)

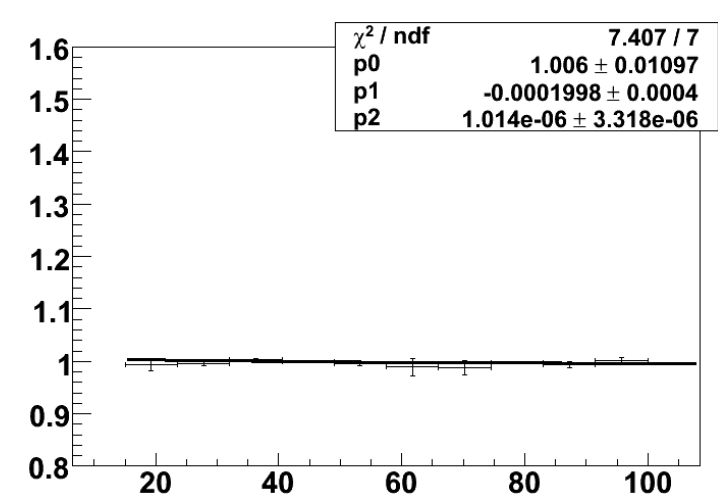

(b)

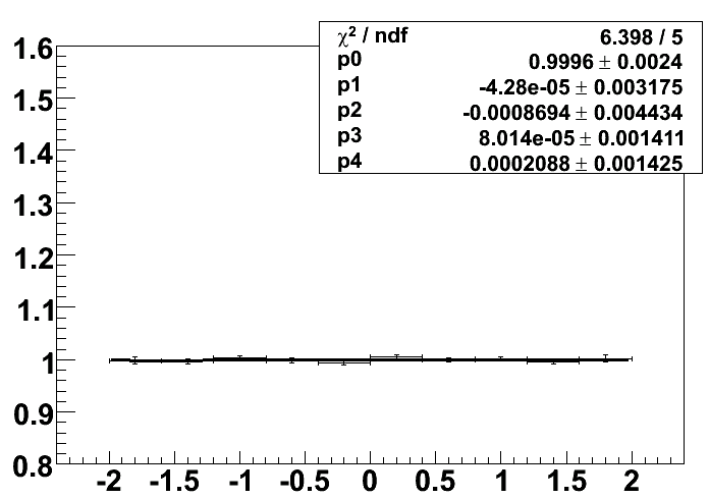

(d)

Figure 36: Closure test in the muon+track channel for (a) loose track scale factor as a function of $p_{T}$ (b) tight track scale factor as a function of $p_{T}$ (c) loose track scale factor as a function of $\eta(\mathrm{d})$ tight track scale factor as a function of $\eta$. 
As the above scale factor calculation used track matched leptons for the tag-and-probe method, an additional scale factor correction is required to correct for the efficiency for a track to be successfully reconstructed. This analysis was performed using a sample of local muons (muons which are reconstructed from only muon system data, which means that no matching to tracks in the central tracker was attempted) and corrects for random overlaps. The technique used is described in [72]. The same track reconstruction scale factor is used for both the electron+track and muon+track channels.

The track reconstruction scale factor was evaluated to be:

$$
\kappa_{\text {reco }}=0.993 \pm 0.025
$$




\section{B Details of the Matrix Method}

The purpose of the matrix method is to determine the three sources of "fake" backgrounds $\left(N_{R L, F T}, N_{F L, R T}, N_{F L, F T}\right)$, as discussed in Section 6.6. To estimate these three quantities, we start with the sample of data events with a loose lepton and a loose track $\left(N_{L L, L T}\right)$ and create three subsamples by tightening the lepton cut $\left(N_{T L, L T}\right)$, the track cut $\left(N_{L L, T T}\right)$ or both $\left(N_{T L, T T}\right)$. Then

$$
\begin{aligned}
& N_{L L, L T}=N_{R L, R T}+N_{F L, R T}+N_{R L, F T}+N_{F L, F T} \\
& N_{T L, L T}=\epsilon_{s i g}^{l e p} N_{R L, R T}+\epsilon_{b k g}^{l e p} N_{F L, R T}+\epsilon_{s i g}^{l e p} N_{R L, F T}+\epsilon_{b k g}^{l e p} N_{F L, F T} \\
& N_{L L, T T}=\epsilon_{s i g}^{t r k} N_{R L, R T}+\epsilon_{s i g}^{t r k} N_{F L, R T}+\epsilon_{b k g}^{t r k} N_{R L, F T}+\epsilon_{b k g}^{t r k} N_{F L, F T} \\
& N_{T L, T T}=\epsilon_{s i g}^{l e p} \epsilon_{s i g}^{t r k} N_{R L, R T}+\epsilon_{b k g}^{l e p} \epsilon_{s i g}^{t r k} N_{F L, R T}+\epsilon_{s i g}^{l e p} \epsilon_{b k g}^{t r k} N_{R L, F T}+\epsilon_{b k g}^{l e p} \epsilon_{b k g}^{t r k} N_{F L, F T}(52)
\end{aligned}
$$

Here $\epsilon_{s i g}^{l e p}\left(\epsilon_{s i g}^{t r k}\right)$ is the efficiency for a real lepton (track) which passes all loose cuts to also pass the tight cut while $\epsilon_{b k g}^{l e p}\left(\epsilon_{b k g}^{t r k}\right)$ is the corresponding efficiency for a fake lepton (track). These efficiencies are determined in Appendix C. Inverting this set of equations gives the desired result

$$
\begin{aligned}
N_{R L, R T} & =\frac{\epsilon_{b k g}^{l e p} \epsilon_{b k g}^{t r k} N_{L L, L T}-\epsilon_{b k g}^{l e p} N_{L L, T T}-\epsilon_{b k g}^{t r k} N_{T L, L T}+N_{T L, T T}}{\left(\epsilon_{s i g}^{l e p}-\epsilon_{b k g}^{l e p}\right)\left(\epsilon_{s i g}^{t r k}-\epsilon_{b k g}^{t r k}\right)} \\
N_{F L, R T} & =\frac{-\epsilon_{s i g}^{l e p} \epsilon_{b k g}^{t r k} N_{L L, L T}+\epsilon_{s i g}^{l e p} N_{L L, T T}+\epsilon_{b k g}^{t r k} N_{T L, L T}-N_{T L, T T}}{\left(\epsilon_{s i g}^{l e p}-\epsilon_{b k g}^{l e p}\right)\left(\epsilon_{s i g}^{t r k}-\epsilon_{b k g}^{t r k}\right)} \\
N_{R L, F T} & =\frac{-\epsilon_{b k g}^{l e p} \epsilon_{s i g}^{t r k} N_{L L, L T}+\epsilon_{b k g}^{l e p} N_{L L, T T}+\epsilon_{s i g}^{t r k} N_{T L, L T}-N_{T L, T T}}{\left(\epsilon_{s i g}^{l e p}-\epsilon_{b k g}^{l e p}\right)\left(\epsilon_{s i g}^{t r k}-\epsilon_{b k g}^{t r k}\right)} \\
N_{F L, F T}= & \frac{\epsilon_{s i g}^{l e p} \epsilon_{s i g}^{t r k} N_{L L, L T}-\epsilon_{s i g}^{l e p} N_{L L, T T}-\epsilon_{s i g}^{t r k} N_{T L, L T}+N_{T L, T T}}{\left(\epsilon_{s i g}^{l e p}-\epsilon_{b k g}^{l e p}\right)\left(\epsilon_{s i g}^{t r k}-\epsilon_{b k g}^{t r k}\right)}
\end{aligned}
$$


A separate matrix method calculation is done for each channel (electron+track and muon+track) and for different jet multiplicity bins (1 jet exclusive and 2 jet inclusive bins). The matrix method is performed on data events that have all selection cuts applied except for the $\not_{T}, E_{T}^{Z-f i t}$, and b-tagging cuts. The real lepton, fake track and fake lepton, real track backgrounds are then esimated by:

- Applying all cuts except for $\not_{T}, E_{T}^{Z-f i t}$, and b-tagging to W Monte Carlo events. W samples were generated using the Alpgen event generator as discussed in Section 6.2 with 0,1,2,3,4, or 5 or more extra light parton jets. Events with two heavy c or b quarks are included as separate samples with 0 to 3 extra light parton jets. A factor of $1.17 \pm 0.15$ is needed to scale the heavy flavor samples relative to the light flavor samples [66].

- Normalizing the W Monte Carlo to the sum $N_{F L, R T}+N_{R L, F T}$.

- Apply the $E_{T}$ and $E_{T}^{Z-f i t}$ cuts.

- Apply the weighting factors for b-tagging to the jets.

Finally the background from fake lepton, fake track events is calculated by:

- The data events which were used in the matrix method calculation (and therefore have no $\not_{T}, E_{T}^{Z-f i t}$, and b-tagging cuts applied) with one loose but not tight lepton and one loose but not tight track are selected. This sample will consist primarily of QCD multijet events.

- This sample of events is normalized to $N_{F L, F T}$.

- The $\#_{T}, \#_{T}^{Z-f i t}$, and b-tagging cuts are applied.

The signal and fake rates, along with their errors, which are used in the matrix method calculation are shown in Table 16 and the number of events in the 
loose and tight samples are shown in Table 17. Table 18 shows the number of real and fake events which are expected in the tight lepton, tight track sample. The number of expected background events is less than zero for two of the entries, but is statistically close to zero.

\begin{tabular}{|c|c|c|c|c|c|c|c|}
\hline \multicolumn{4}{|l|}{ e+track } & \multicolumn{4}{|l|}{$\mu+$ track } \\
\hline & Eff. & Stat & Syst & & Eff. & Stat & Err. Syst \\
\hline 1 jet bin & & & & 1 jet bin & & & \\
\hline$\epsilon_{s i g}^{e}$ & 0.868 & 0.004 & 0.053 & $\epsilon_{s i g}^{\mu}$ & 0.936 & 0.005 & 0.051 \\
\hline$\epsilon_{s i g}^{t r k}$ & 0.904 & 0.006 & 0.082 & $\epsilon_{s i g}^{t r k}$ & 0.915 & 0.007 & 0.093 \\
\hline$\epsilon_{b k g}^{e}$ & 0.119 & $<0.001$ & $\begin{array}{l}+0.022 \\
{ }_{-0.010}\end{array}$ & $\epsilon_{b k g}^{\mu k g}$ & 0.063 & 0.002 & $\begin{array}{l}+0.028 \\
{ }_{-0.008}\end{array}$ \\
\hline$\epsilon_{b k g}^{t r k}$ & 0.287 & 0.001 & $\begin{array}{l}+0.056 \\
-0.057\end{array}$ & $\epsilon_{b k g}^{t r k}$ & 0.336 & 0.012 & $\begin{array}{l}+0.091 \\
{ }_{-0.059}\end{array}$ \\
\hline 2 jet bin & & & & 2 jet bin & & & \\
\hline$\epsilon_{s i q}^{e}$ & 0.859 & 0.005 & 0.021 & $\epsilon_{s i q}^{\mu}$ & 0.893 & 0.005 & 0.015 \\
\hline$\epsilon_{s i g}^{t r k}$ & 0.885 & 0.008 & 0.038 & $\epsilon_{s i g}^{t r k}$ & 0.866 & 0.007 & 0.019 \\
\hline$\epsilon_{b k g}^{e}$ & 0.145 & 0.001 & $\begin{array}{l}+0.024 \\
-0.015\end{array}$ & $\epsilon_{b k q}^{\mu}$ & 0.083 & 0.003 & $\begin{array}{l}+0.019 \\
-0.009\end{array}$ \\
\hline$\epsilon_{b k q}^{t r k g}$ & 0.314 & 0.003 & $\begin{array}{l}+0.058 \\
-0.059\end{array}$ & $\epsilon_{b k q}^{t r k}$ & 0.330 & 0.011 & $\begin{array}{l}+0.065 \\
-0.080\end{array}$ \\
\hline
\end{tabular}

Table 16: Table of signal and fake rates used in the matrix method background estimate.

\begin{tabular}{|c|c|c|c|}
\hline \multicolumn{2}{|l|}{ e+track } & \multicolumn{2}{|l|}{$\mu+$ track } \\
\hline & nber & & mber \\
\hline 1 jet bin & & 1 jet bin & \\
\hline $\mathrm{N}_{L L, L T}$ & 989 & $\mathrm{~N}_{L L, L T}$ & 717 \\
\hline $\mathrm{N}_{T L, L T}$ & 602 & $\mathrm{~N}_{T L, L T}$ & 605 \\
\hline $\mathrm{N}_{L L, T T}$ & 664 & $\mathrm{~N}_{L L, T T}$ & 611 \\
\hline $\mathrm{N}_{T L, T T}$ & 499 & $\mathrm{~N}_{T L, T T}$ & 542 \\
\hline 2 jet bin & & 2 jet bin & \\
\hline $\mathrm{N}_{L L, L T}$ & 376 & $\mathrm{~N}_{L L, L T}$ & 332 \\
\hline $\mathrm{N}_{T L, L T}$ & 241 & $\mathrm{~N}_{T L, L T}$ & 273 \\
\hline $\mathrm{N}_{L L, T T}$ & 224 & $\mathrm{~N}_{L L, T T}$ & 281 \\
\hline $\mathrm{N}_{T L, T T}$ & 175 & $\mathrm{~N}_{T L, T T}$ & 243 \\
\hline
\end{tabular}

Table 17: Number of events in the loose and tight samples used for background estimation in electron+track and muon+track channels. 


\begin{tabular}{|c|c|c|c|c|c|}
\hline \multicolumn{3}{|l|}{$\mathrm{e}+$ track } & \multicolumn{3}{|l|}{$\mu+$ track } \\
\hline & Number & Stat. Err. & & amber & Stat. Err. \\
\hline 1 jet bin & & & 1 jet bin & & \\
\hline $\mathrm{N}_{F L, F T}$ & 11.4 & 0.9 & $\mathrm{~N}_{F L, F T}$ & 1.3 & 0.2 \\
\hline $\mathrm{N}_{R L, F T}$ & 9.5 & 4.6 & $\mathrm{~N}_{R L, F T}$ & 5.3 & 4.4 \\
\hline $\mathrm{N}_{F L, R T}$ & 0.9 & 2.1 & $\mathrm{~N}_{F L, R T}$ & 0.9 & 0.6 \\
\hline $\mathrm{N}_{R L, R T}$ & 477.2 & 24.3 & $\mathrm{~N}_{R L, R T}$ & 534.5 & 24.9 \\
\hline 2 jet bin & & & 2 jet bin & & \\
\hline $\mathrm{N}_{F L, F T}$ & 6.1 & 0.8 & $\mathrm{~N}_{F L, F T}$ & 0.8 & 0.2 \\
\hline $\mathrm{N}_{R L, F T}$ & 14.8 & 4.2 & $\mathrm{~N}_{R L, F T}$ & -4.9 & 3.2 \\
\hline $\mathrm{N}_{F L, R T}$ & -2.6 & 1.6 & $\mathrm{~N}_{F L, R T}$ & 0.03 & 0.7 \\
\hline $\mathrm{N}_{R L, R T}$ & 156.6 & 15.1 & $\mathrm{~N}_{R L, R T}$ & 247.1 & 17.3 \\
\hline
\end{tabular}

Table 18: Number of real and fake events expected in the tight lepton, tight track sample with their statistical errors. 


\section{Efficiencies for the Matrix Method Calculation}

The efficiencies $\epsilon_{s i g}^{e}, \epsilon_{s i g}^{\mu}, \epsilon_{s i g}^{t r k}$ for real tracks and leptons to pass the tight identification cut are determined using $\mathrm{Z} \rightarrow$ ee and $\mathrm{Z} \rightarrow \mu \mu$ Monte Carlo. The Monte Carlo sample used to make this determination is the same as the sample used for the $\mathrm{Z}$ background determination (Section 6.4). The efficiency is taken as the number of loose leptons (or tracks) which pass the tight cut divided by the total number of loose leptons (or tracks), with the Monte Carlo to data scale factors for the tight cut applied after the tight cut is taken. A separate signal efficiency is determined for the electron+track and muon+track channels, using either $\mathrm{Z} \rightarrow$ ee events for electron+track or $\mathrm{Z} \rightarrow \mu \mu$ for muon+track. The efficiency for a real electron to pass the tight likelihood cut is shown in Figure 37. The efficiency for a real muon to pass the muon isolation cuts is shown in Figure 39. The efficiency for a track from a real lepton to pass the track isolation cut is shown in Figure 38 for electron tracks and Figure 40 for muon tracks. All results shown are after the scale factor for the tight cuts has been applied $([73],[74])$. A systematic error on the efficiency is determined by calculating the efficiency on a tét Monte Carlo sample and taking the difference between the $t \bar{t}$ results and the $\mathrm{Z} \rightarrow 1 \mathrm{l}$ results as the systematic error.

The efficiency for a fake electron, muon or track to pass the tight cut must be determined from fake events in data. To obtain a sample of nearly pure fake leptons, all electron+track or muon+track selection cuts are applied except

- No isolated track is required

- $E_{T}$ cut is changed to $\not_{T}<15 \mathrm{GeV}$

- No $\#_{T}^{Z-f i t}$ cut 


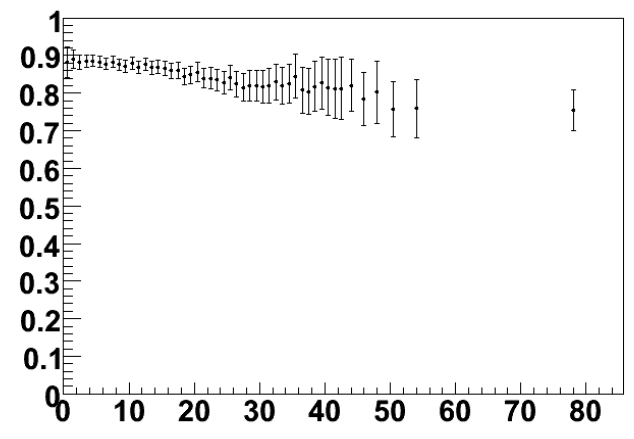

(a)

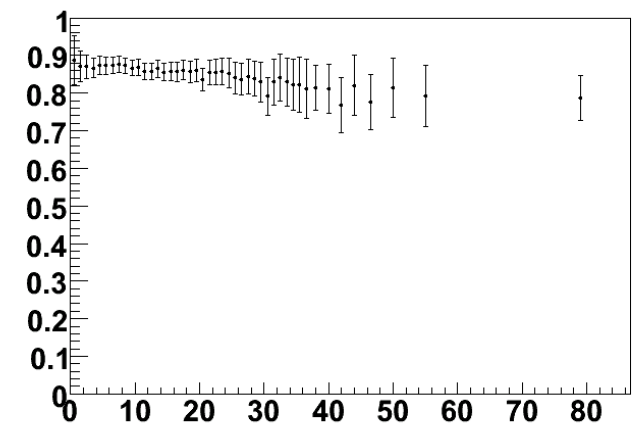

(b)

Figure 37: $\epsilon_{\text {sig }}^{e}$ in the electron+track channel vs $\not_{T}$ for (a) the 1 jet bin and (b) the 2 jet inclusive bin.

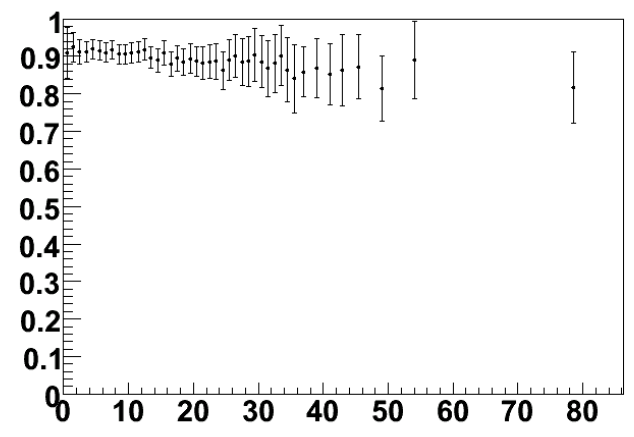

(a)

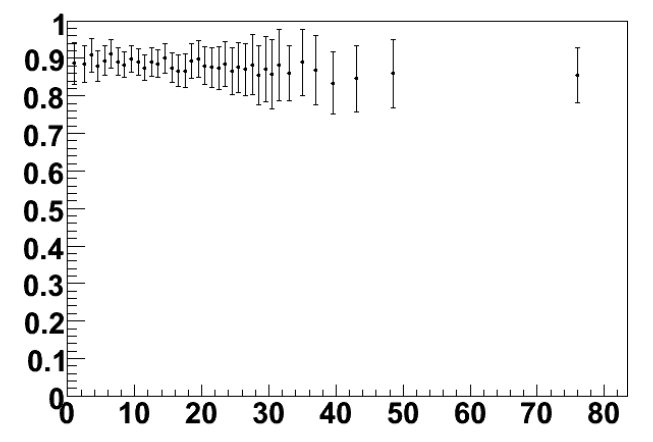

(b)

Figure 38: $\epsilon_{\text {sig }}^{t r k}$ in the electron+track channel vs $\not_{T}$ for (a) the 1 jet bin and (b) the 2 jet inclusive bin.

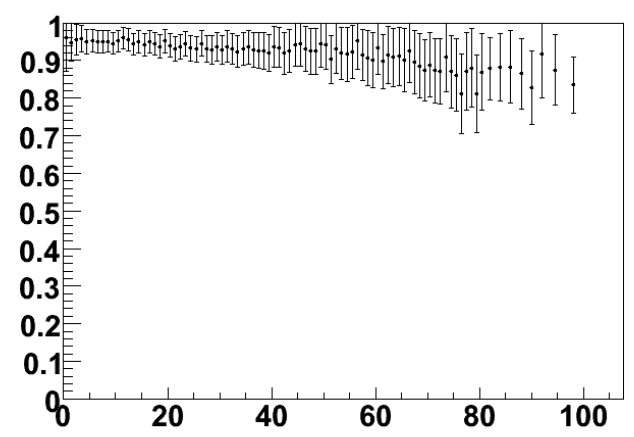

(a)

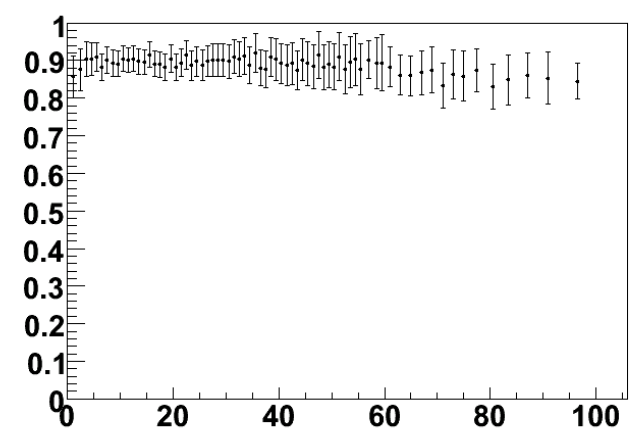

(b)

Figure 39: $\epsilon_{\text {sig }}^{\mu}$ in the muon+track channel vs $E_{T}$ for (a) the 1 jet bin and (b) the 2 jet inclusive bin. 


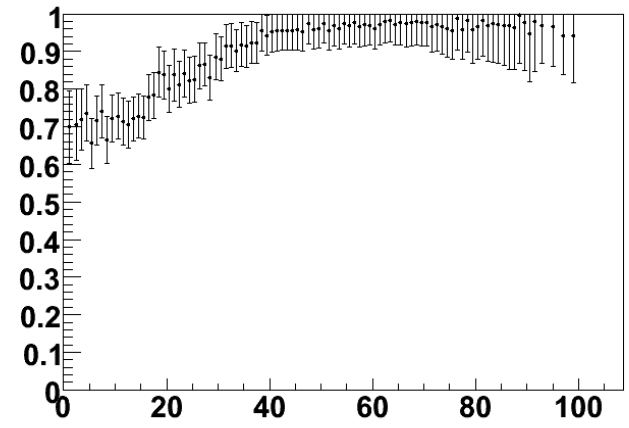

(a)

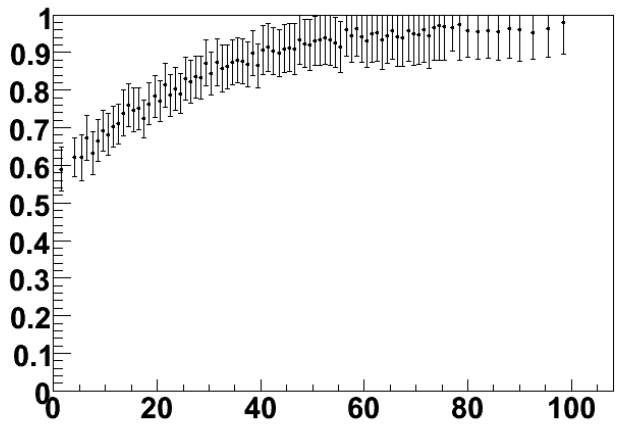

(b)

Figure 40: $\epsilon_{s i g}^{t r k}$ in the muon+track channel vs $E_{T}$ for (a) the 1 jet bin and (b) the 2 jet inclusive bin

- $\not_{T j e t}<25 \mathrm{GeV} . E_{T}$ jet is the missing transverse energy with only jet energy corrections and no muon or electron corrections.

- A Z veto cut is applied which requires that the lepton does not have a mass of 70-100 GeV with another lepton or a track.

The efficiency is calculated by dividing the number of tight leptons by the total number of loose leptons for events with $\not_{T}<15 \mathrm{GeV}$. The resulting efficiency for a fake electron is shown in Figure 41 and the fake muon results are shown in Figure 43. A systematic error on the efficiency is estimated by doing a quadratic fit to the efficiency vs $\not_{T}$ plot and taking the minimum and maximum of the fit in the range from 0 to $15 \mathrm{GeV}$.

The track samples are defined similarly: all selection cuts are applied except

- No identified lepton is required

- $E_{T}$ cut is changed to $\#_{T}<15 \mathrm{GeV}$

- No $B_{T}^{Z-f i t}$ cut

- $\mathbb{E}_{T \text { jet }}<25 \mathrm{GeV}$ 


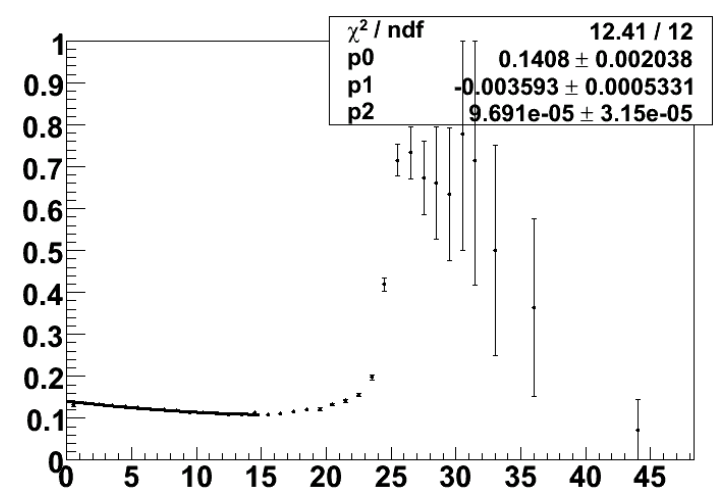

(a)

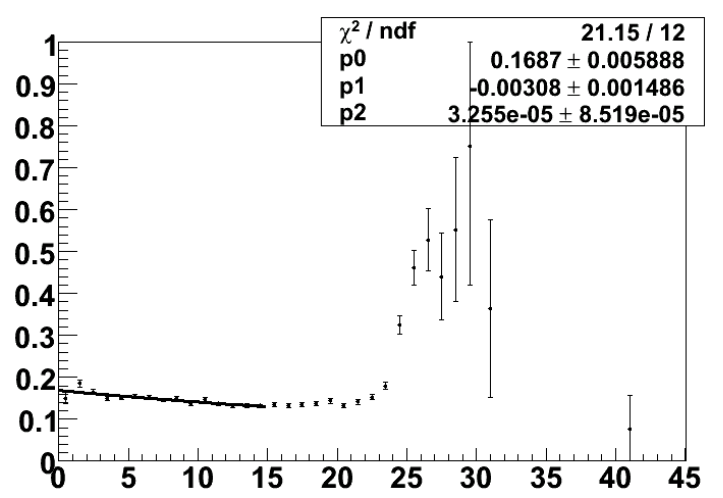

(b)

Figure 41: $\epsilon_{b k g}^{e}$ in the electron+track channel vs $\not_{T}$ for (a) the 1 jet bin and (b) the 2 jet inclusive bin. Only data points up to $E_{T}$ of $15 \mathrm{GeV}$ are used for the efficiency calculation. The quadratic fit is used for systematic error estimation.

- The requirement $\Delta z$ (track, lepton vertex $)<1 \mathrm{~cm}$ is replaced by $\Delta z($ track, jet vertex $)<1 \mathrm{~cm}$.

- A Z veto cut is applied which requires that the track does not have a mass of 70 to $100 \mathrm{GeV}$ with a lepton.

The efficiency is the number of tight tracks divided by the total number of loose tracks for events with $E_{T}<15 \mathrm{GeV}$. The efficiency for a fake track in electron+track is shown in Figure 42 and muon+track in Figure 44. A quadratic fit of the efficiency versus $Z_{T}$ is also used for the track efficiencies to estimate the systematic error.

The efficiency of the $\mathrm{D} \varnothing$ tracking system is strongly dependent upon occupancy, and therefore efficiency is luminosity dependent as well. The fake efficiency is plotted as a function of instantaneous luminosity in Figures 45 and 46. This depedence on luminosity is estimated from a linear fit, and half the difference between the values of this fit at luminosities of $0 \times 10^{30} \mathrm{~cm}^{-2} \mathrm{~s}^{-1}$ and $150 \times 10^{30} \mathrm{~cm}^{-2} \mathrm{~s}^{-1}$ is taken as an additional source of systematic error. 


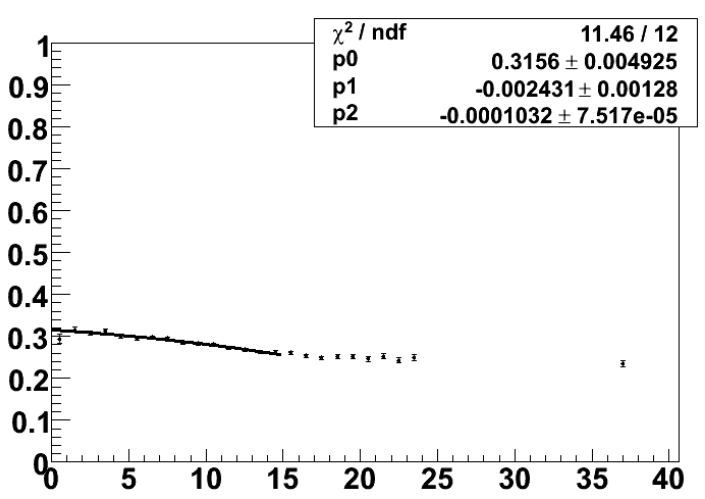

(a)

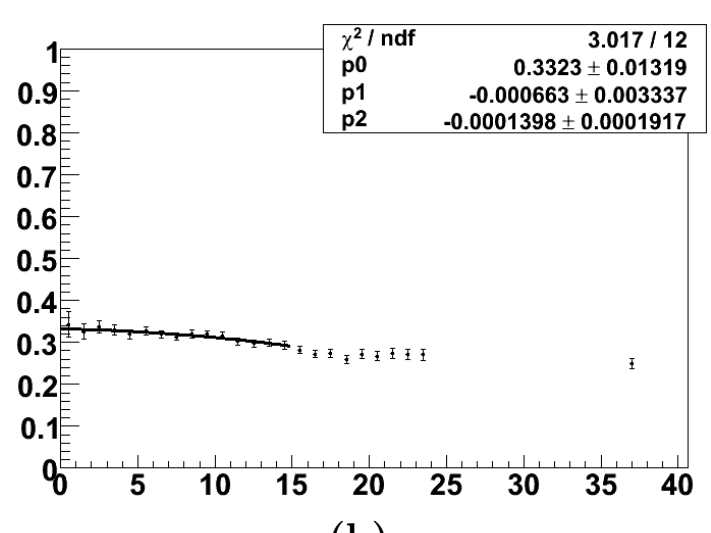

(b)

Figure 42: $\epsilon_{b k g}^{t r k}$ in the electron+track channel vs $E_{T}$ for (a) the 1 jet bin and (b) the 2 jet inclusive bin. Only data points up to $E_{T}$ of $15 \mathrm{GeV}$ are used for the efficiency calculation. The quadratic fit is used for systematic error estimation.

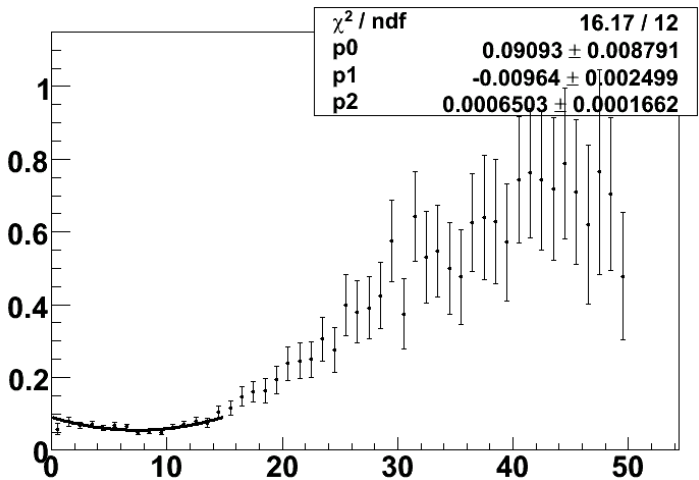

(a)

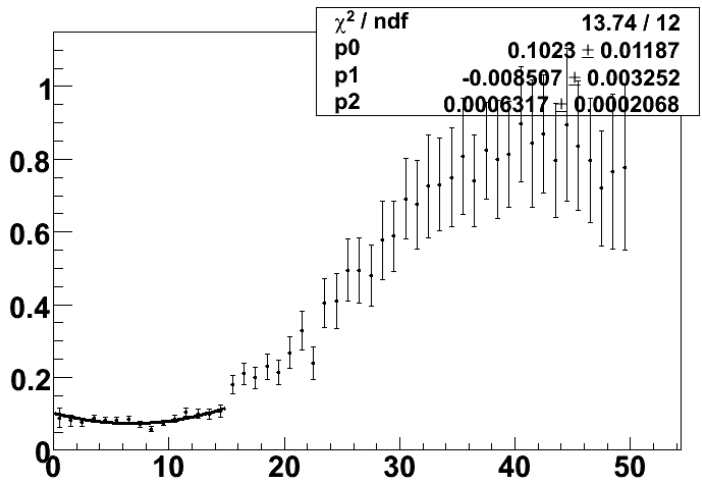

(b)

Figure 43: $\epsilon_{b k g}^{\mu}$ in the muon+track channel vs $E_{T}$ for (a) the 1 jet bin and (b) the 2 jet inclusive bin. Only data points up to $E_{T}$ of $15 \mathrm{GeV}$ are used for the efficiency calculation. The quadratic fit is used for systematic error estimation.

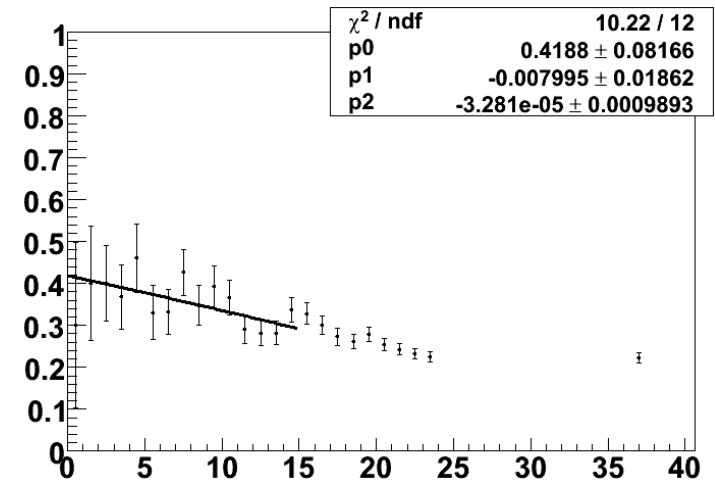

(a)

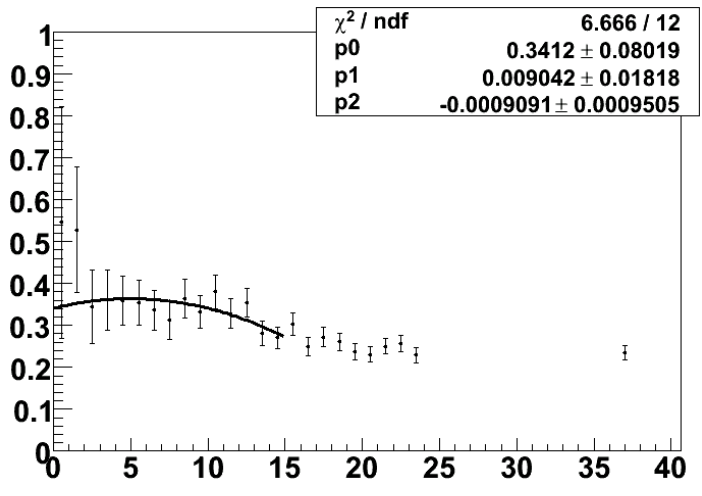

(b)

Figure 44: $\epsilon_{b k g}^{t r k}$ in the muon+track channel vs $E_{T}$ for (a) the 1 jet bin and (b) the 2 jet inclusive bin. Only data points up to $E_{T}$ of $15 \mathrm{GeV}$ are used for the efficiency calculation. The quadratic fit is used for systematic error estimation. 


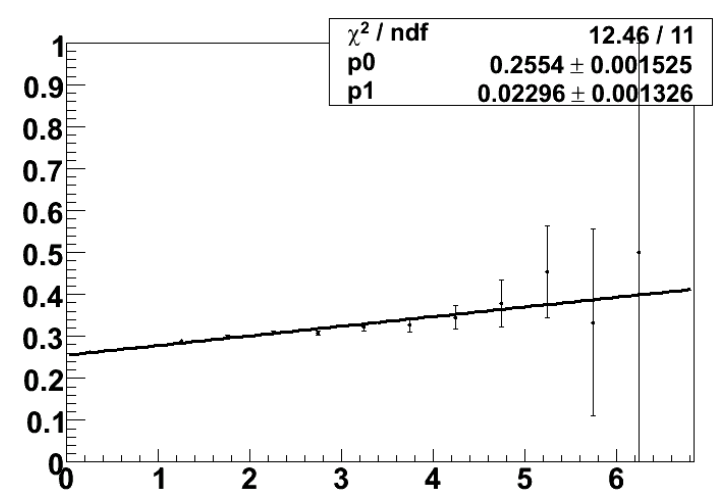

(a)

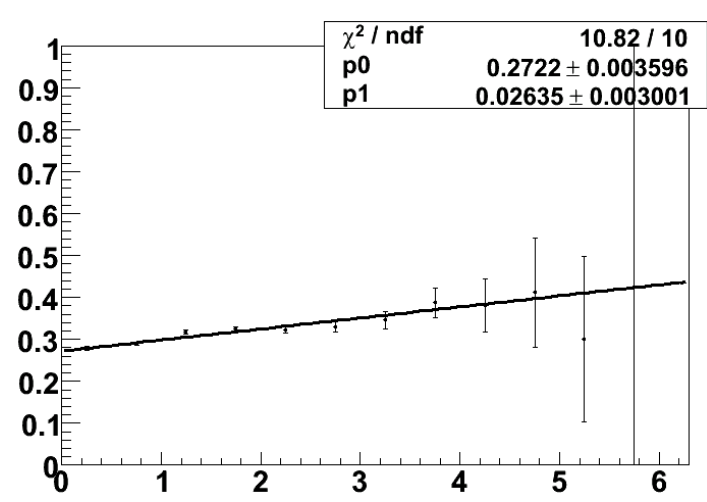

(b)

Figure 45: $\epsilon_{b k g}^{t r k}$ in the electron+track channel vs instantaneous luminosity for (a) the 1 jet bin and (b) the 2 jet inclusive bin. The linear fit is used for systematic error estimation.

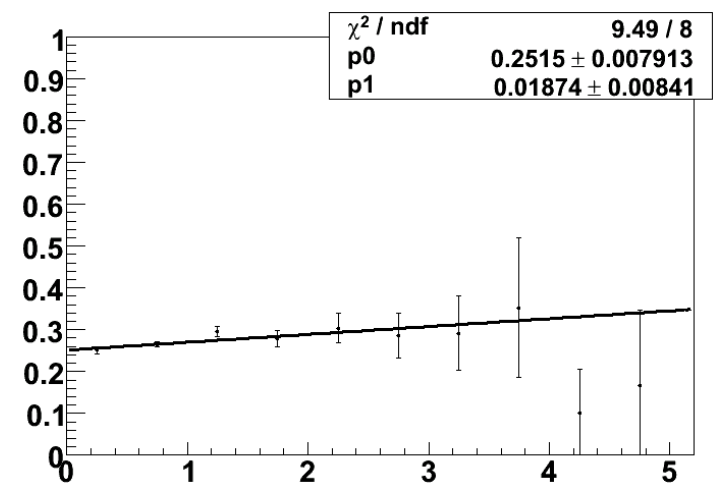

(a)

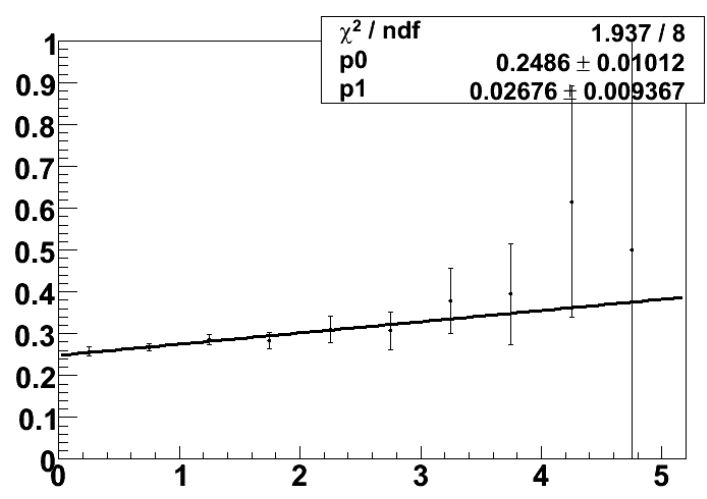

(b)

Figure 46: $\epsilon_{b k g}^{t r k}$ in the muon+track channel vs instantaneous luminosity for (a) the 1 jet bin and (b) the 2 jet inclusive bin. The linear fit is used for systematic error estimation. 


\section{Control Plots}

Figures 47-66 display a variety of kinematic distributions in the electron+track channel, and Figures 67-86 show the same distributions in the muon+track channel. In all figures the top row is the one jet bin, and the bottom row is the two (or more) jet bin (the only exceptions are the distributions for the second leading jet, where there of course is no one jet bin). Four samples with different selection cuts are displayed for each variable: no missing momentum or b-tagging cuts, just the missing momentum cut, just the b-tagging cut, and finally the sample with all cuts applied, which is used for the final cross section analysis. Monte Carlo to data agreement is quite good in all cases, indicating that the missing momentum and b-tagging cuts are well-modelled by the simulation.

No $\#_{T}$ or tagging $\#_{T}$ and $\#_{T}^{Z-f i t}$ only Tagging only $\#_{T}, E_{T}^{Z-f i t}$, and tagging
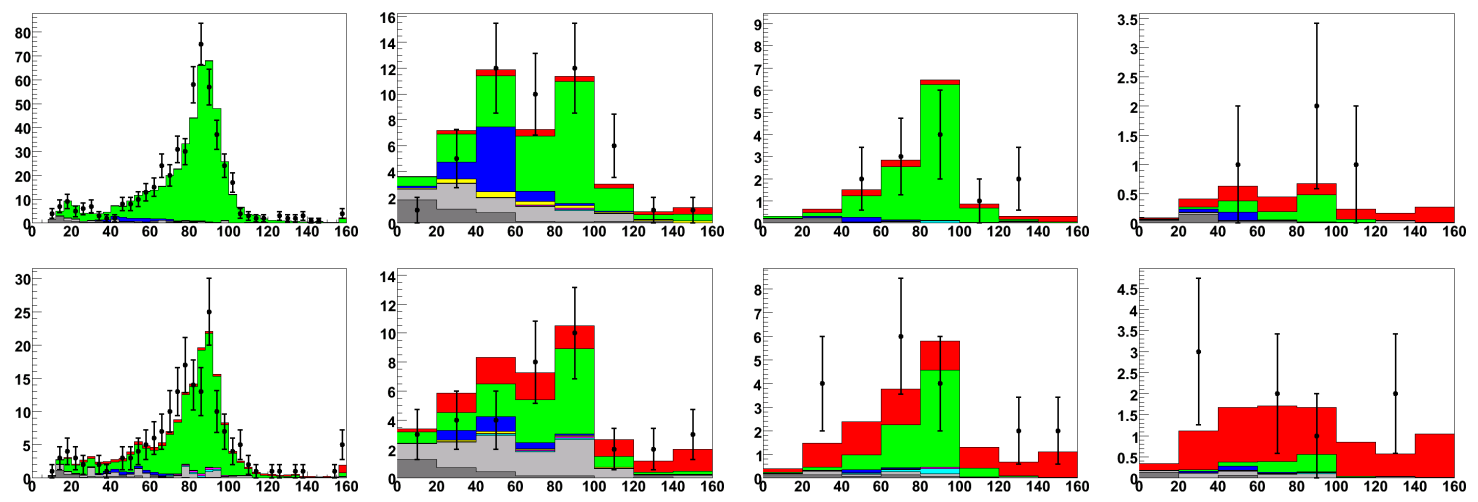

Figure 47: Mass (in $\mathrm{GeV}$ ) of electron-track pair in electron+track.

$\square$ tt $\quad \mathrm{Z} \rightarrow$ ee $\square \mathrm{Z} \rightarrow \tau \tau \quad$ WW $\quad$ WZ $\quad$ ZZ $\quad$ W+Fake $\quad$ Fake+Fake


No $Z_{T}$ or tagging $Z_{T}$ and $\#_{T}^{Z-f i t}$ only Tagging only $\#_{T}, Z_{T}^{Z-f i t}$, and tagging
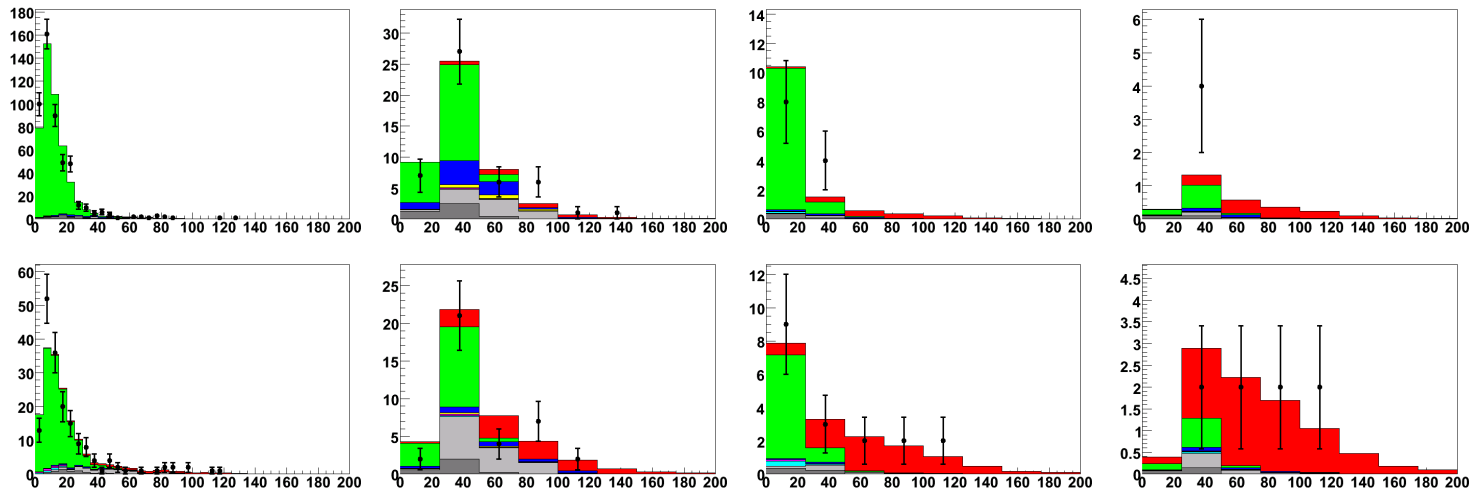

Figure 48: $\quad E_{T}$ (in $\mathrm{GeV}$ ) in electron+track.

No $\#_{T}$ or tagging $\#_{T}$ and $\#_{T}^{Z-f i t}$ only Tagging only $\#_{T}, \#_{T}^{Z-f i t}$, and tagging
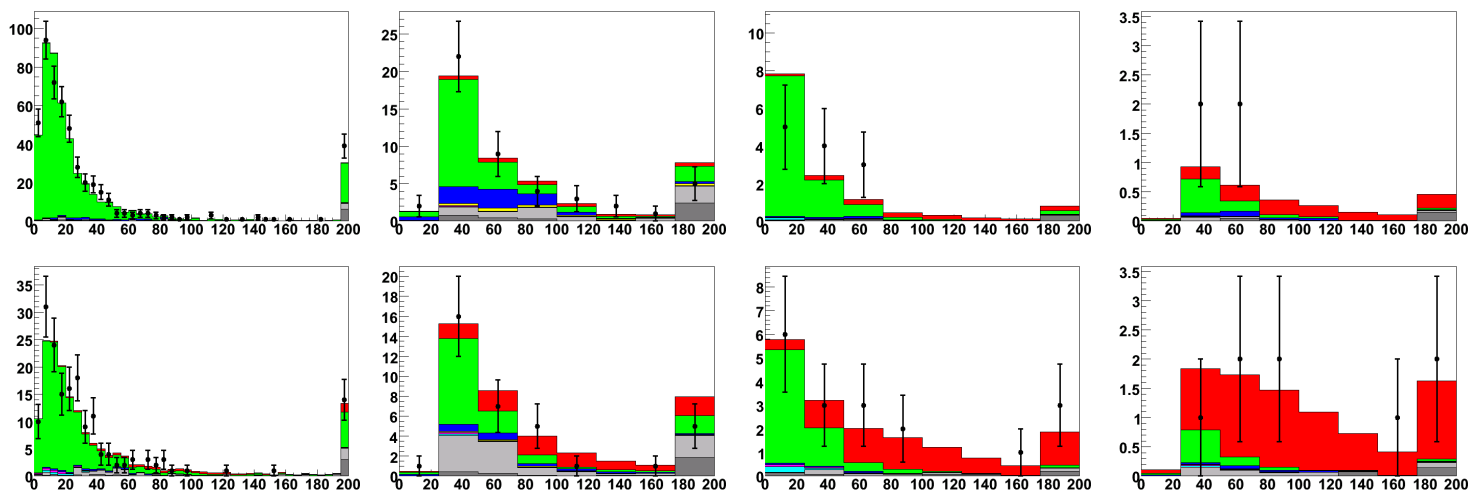

Figure 49: $\quad \not_{T}^{Z-f i t}($ in $\mathrm{GeV})$ in electron+track.
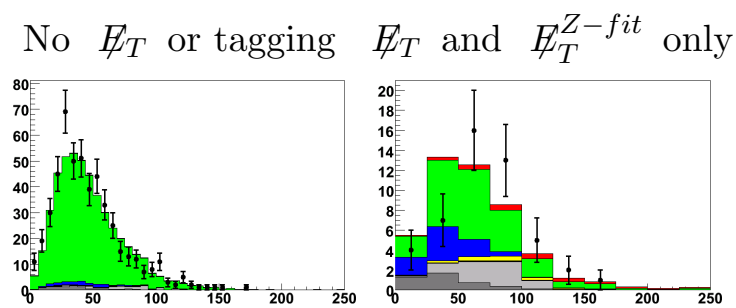

Tagging only

$E_{T}, E_{T}^{Z-f i t}$, and tagging
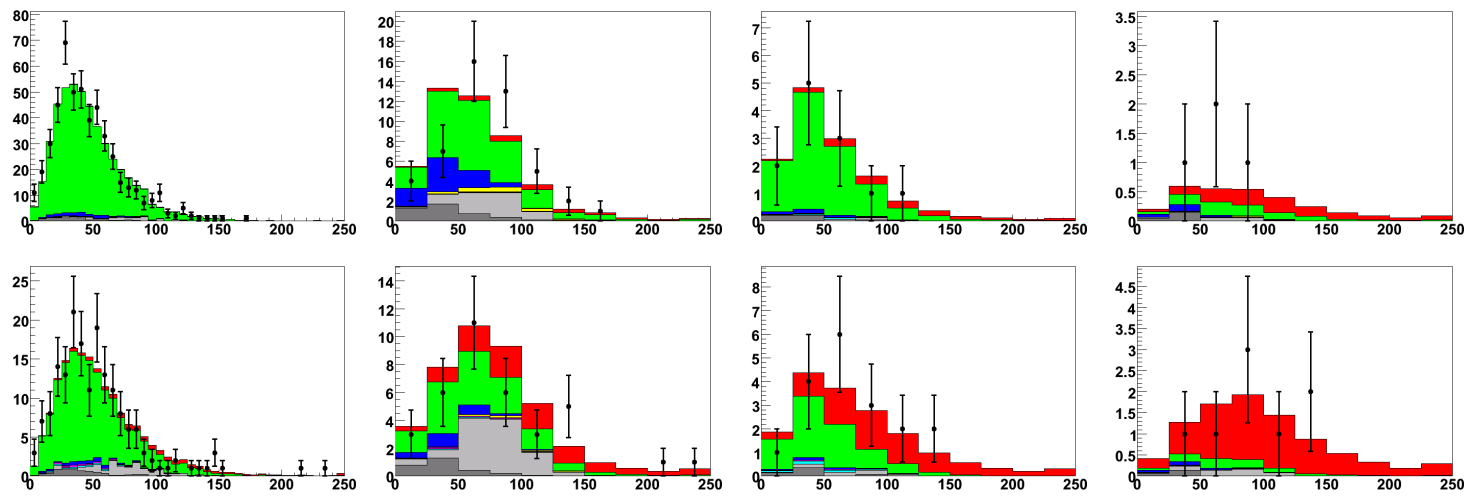

Figure 50: Transverse mass (in $\mathrm{GeV}$ ) of electron and $E_{T}$ in electron+track.

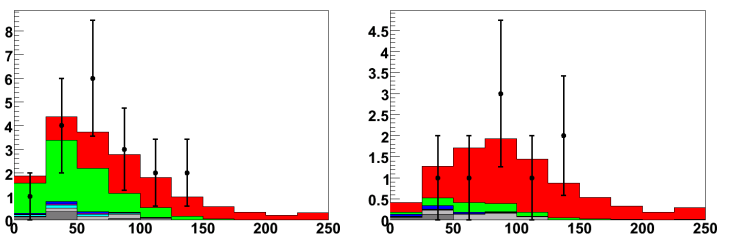

\section{$\square$ tt $\quad \mathrm{Z} \rightarrow \mathrm{ee} \square \mathrm{Z} \rightarrow \tau \tau \quad$ WW $\square$ WZ $\quad$ ZZ $\quad$ W+Fake $\square$ Fake+Fake}


No $\#_{T}$ or tagging $Z_{T}$ and $\#_{T}^{Z-f i t}$ only Tagging only $E_{T}, \#_{T}^{Z-f i t}$, and tagging
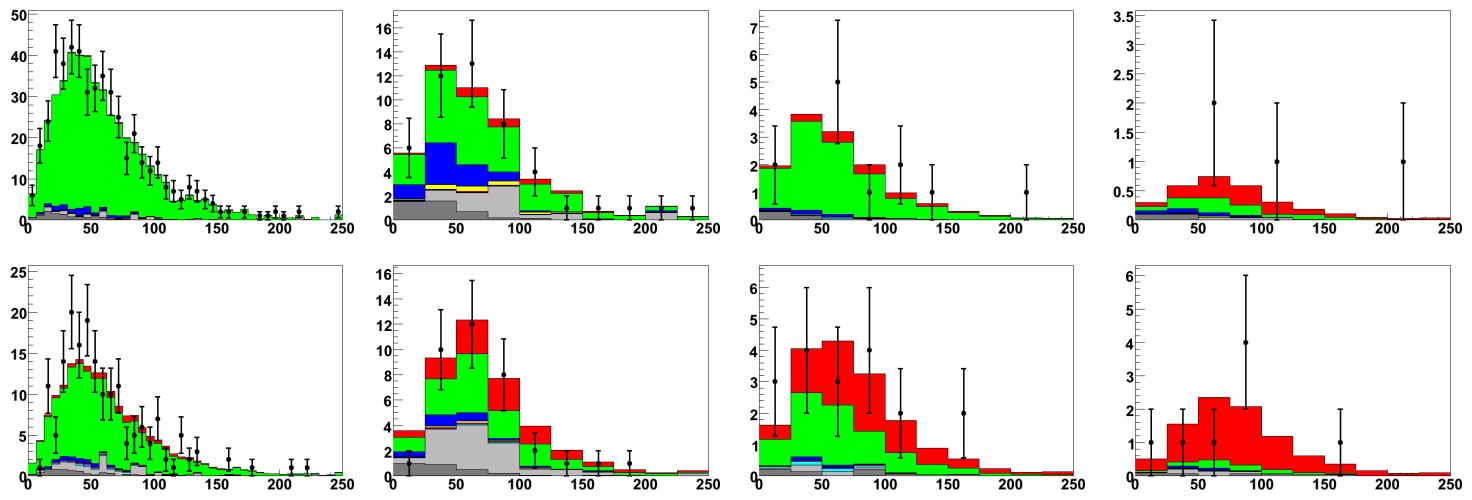

Figure 51: Transverse mass (in $\mathrm{GeV}$ ) of track and $Z_{T}$ in electron+track.

No $\#_{T}$ or tagging $Z_{T}$ and $Z_{T}^{Z-f i t}$ only Tagging only $\#_{T}, \#_{T}^{Z-f i t}$, and tagging
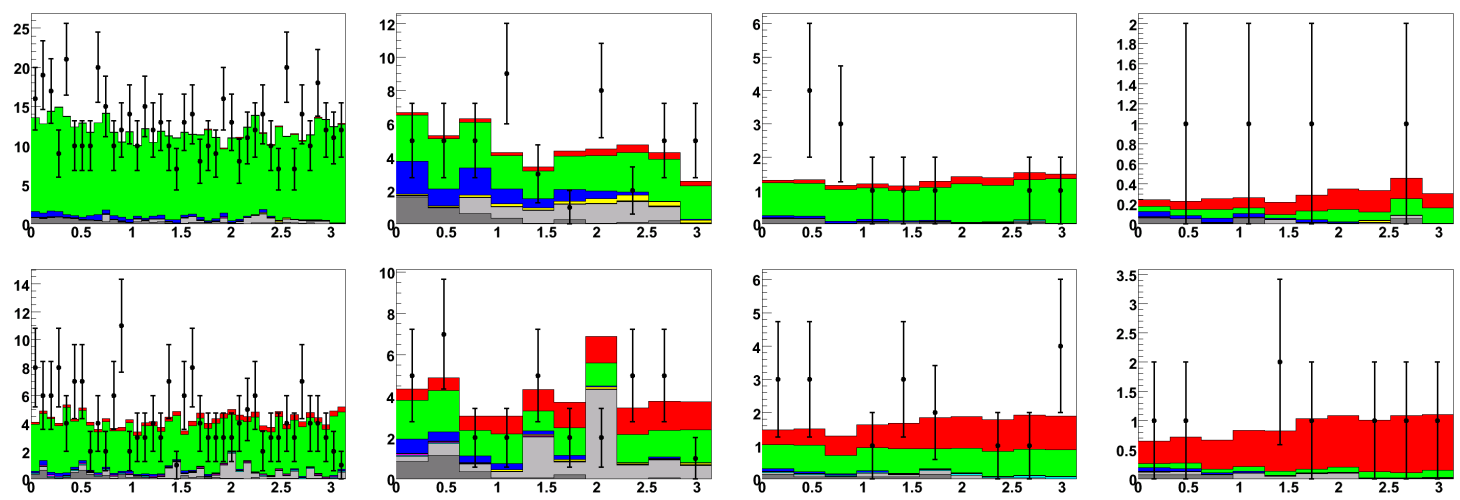

Figure 52: $\Delta \phi$ of electron and $Z_{T}$ in electron+track.

No $\#_{T}$ or tagging $\#_{T}$ and $E_{T}^{Z-f i t}$ only Tagging only $\#_{T}, \#_{T}^{Z-f i t}$, and tagging
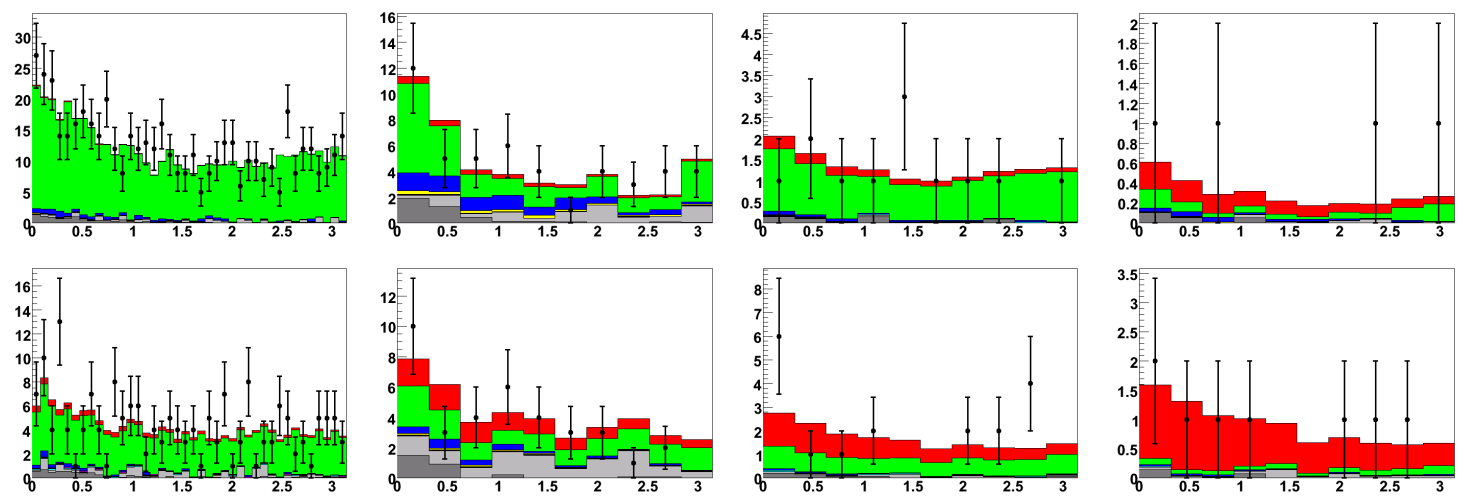

Figure 53: $\Delta \phi$ of track and $\#_{T}$ in electron+track.

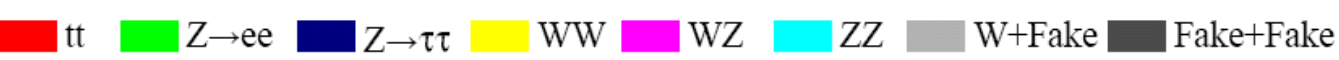


No $\#_{T}$ or tagging $Z_{T}$ and $\#_{T}^{Z-f i t}$ only Tagging only $Z_{T}, E_{T}^{Z-f i t}$, and tagging
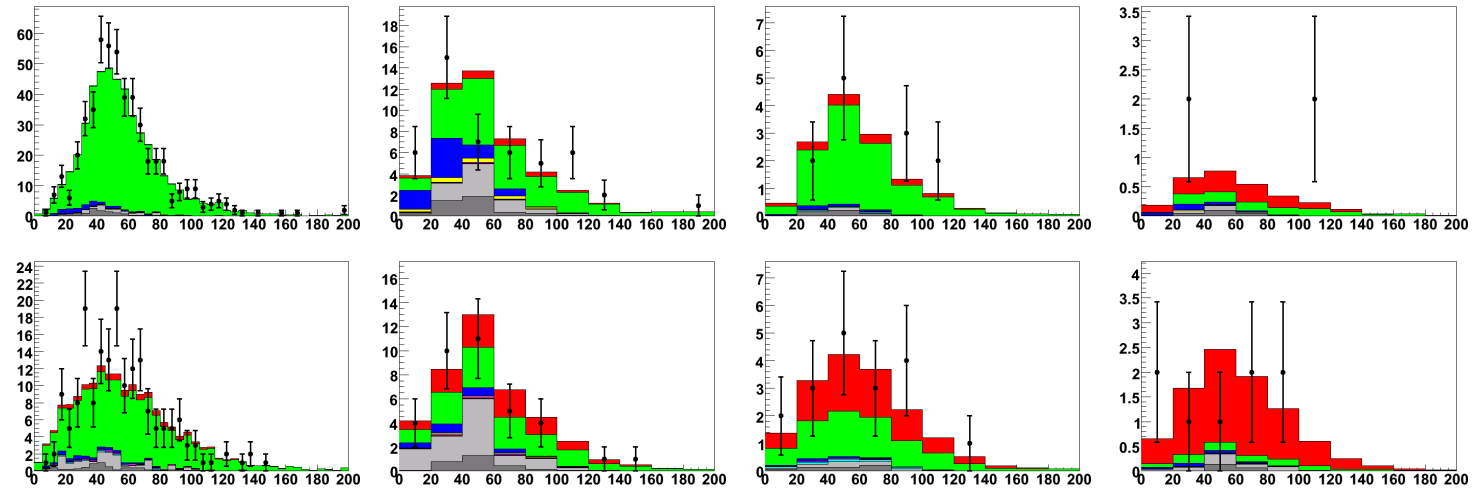

Figure 54: $\mathrm{Z} p_{T}$ (vector sum of electron $p_{T}$ and track $p_{T}$, in $\mathrm{GeV}$ ) in electron+track.

No $E_{T}$ or tagging $\#_{T}$ and $E_{T}^{Z-f i t}$ only Tagging only $E_{T}, E_{T}^{Z-f i t}$, and tagging
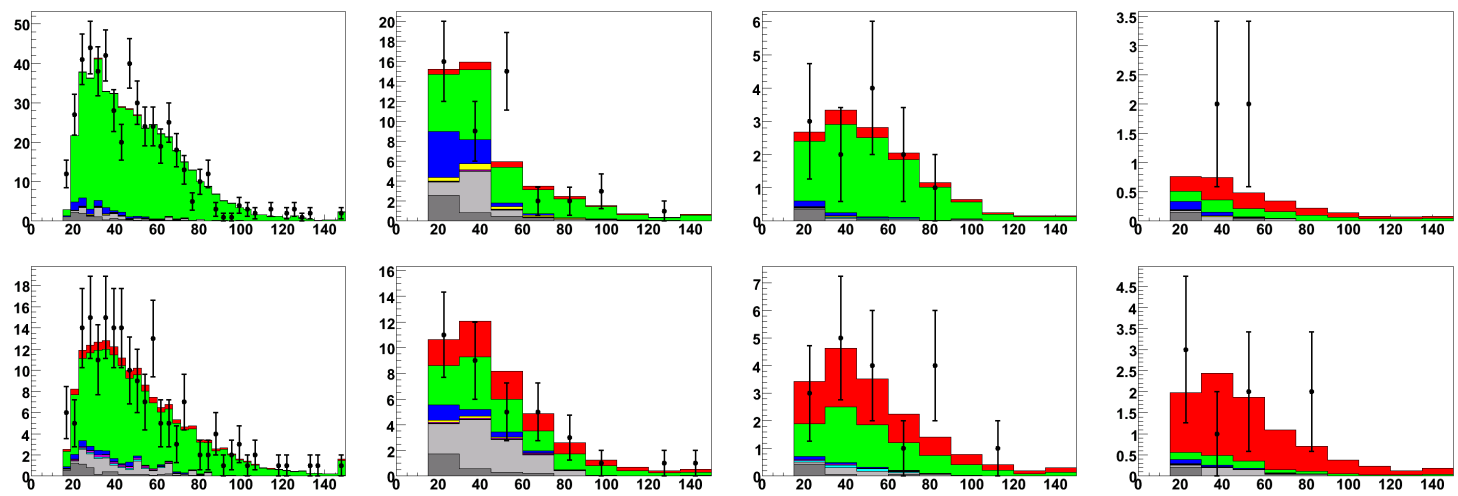

Figure 55: Electron $p_{T}$ (in $\mathrm{GeV}$ ) in electron+track.

No $\#_{T}$ or tagging $\#_{T}$ and $\#_{T}^{Z-f i t}$ only Tagging only $\#_{T}, \#_{T}^{Z-f i t}$, and tagging
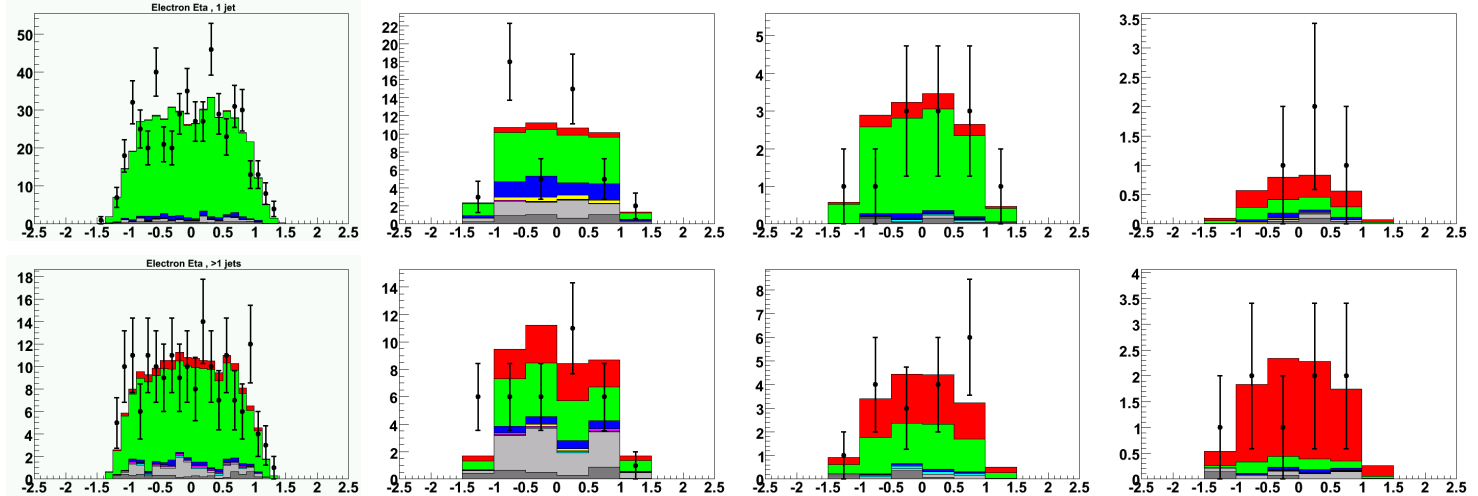

Figure 56: Electron $\eta$ in electron+track. 
No $E_{T}$ or tagging $E_{T}$ and $E_{T}^{Z-f i t}$ only
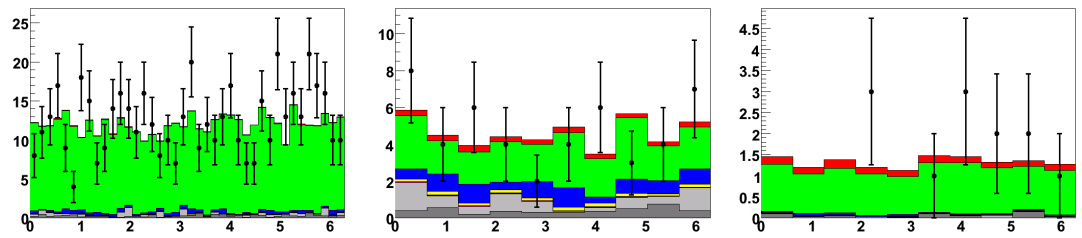

$\#_{T}, \#_{T}^{Z-f i t}$, and tagging
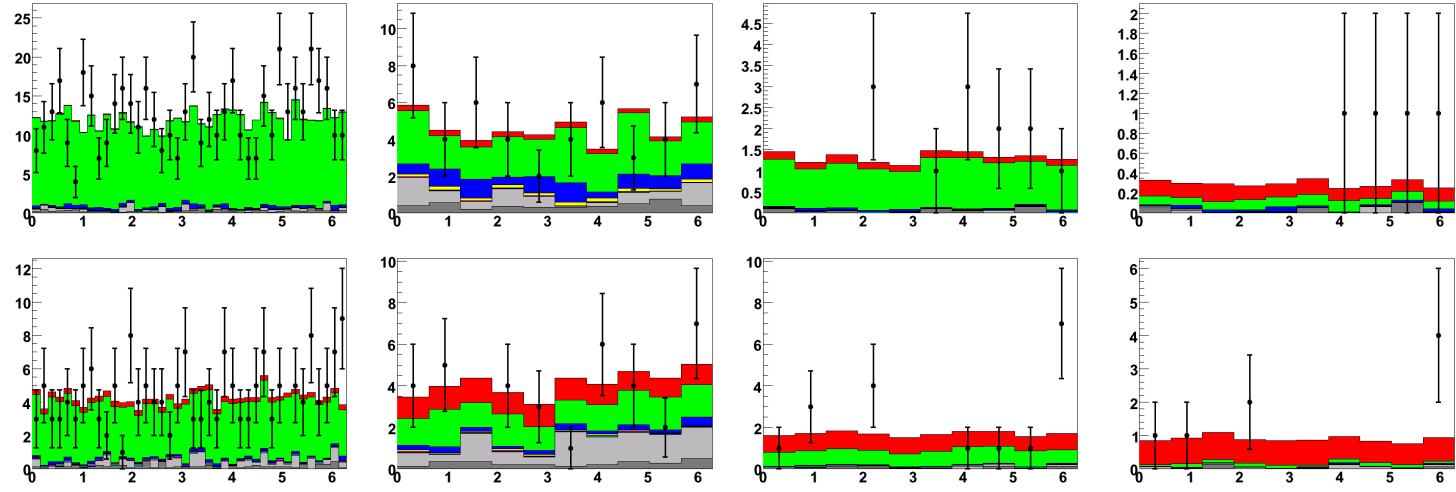

Figure 57: Electron $\phi$ in electron+track.

No $E_{T}$ or tagging $E_{T}$ and $E_{T}^{Z-f i t}$ only Tagging only $E_{T}, E_{T}^{Z-f i t}$, and tagging
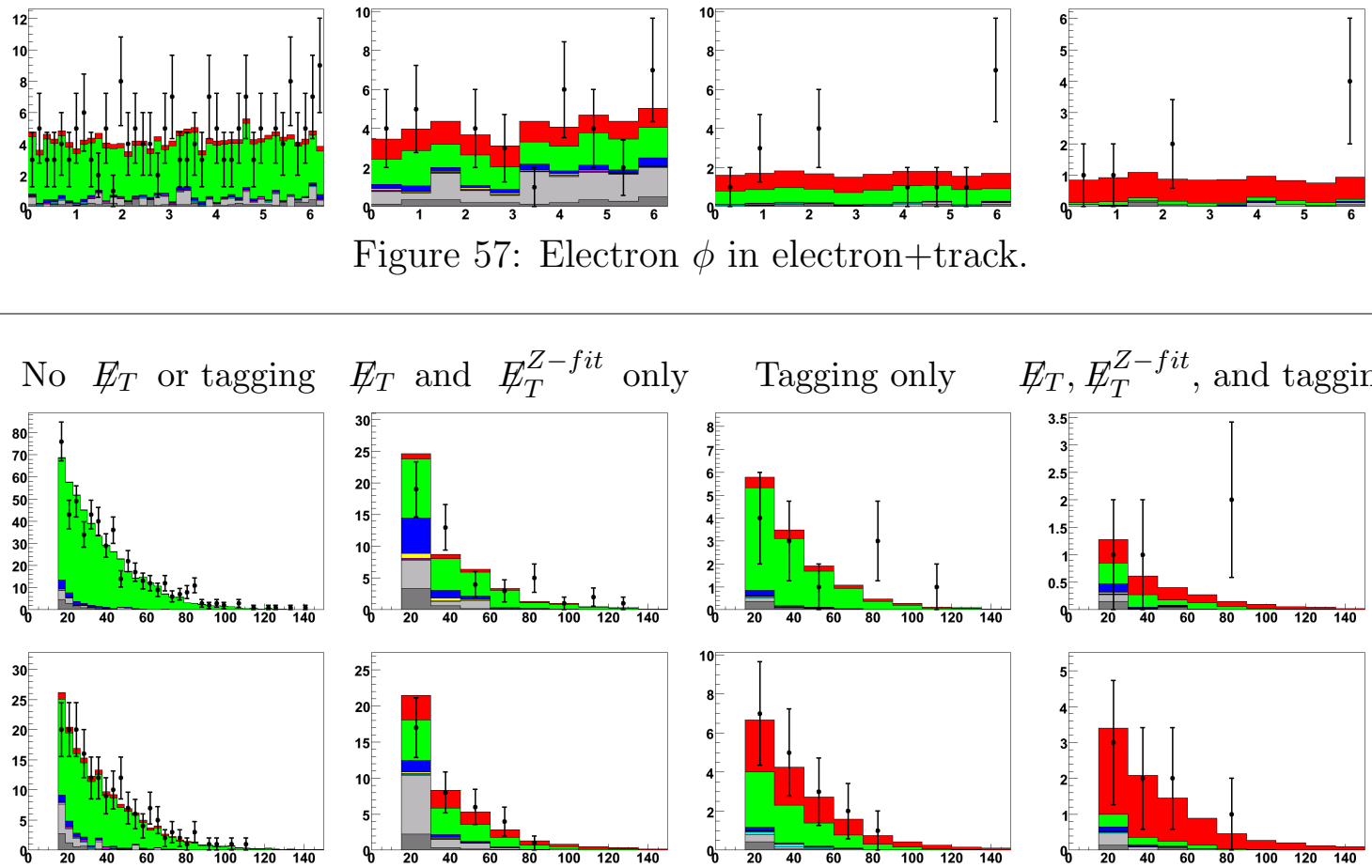

Figure 58: Track $p_{T}$ (in $\mathrm{GeV}$ ) in electron+track.

No $\#_{T}$ or tagging $\#_{T}$ and $E_{T}^{Z-f i t}$ only Tagging only $E_{T}, \#_{T}^{Z-f i t}$, and tagging
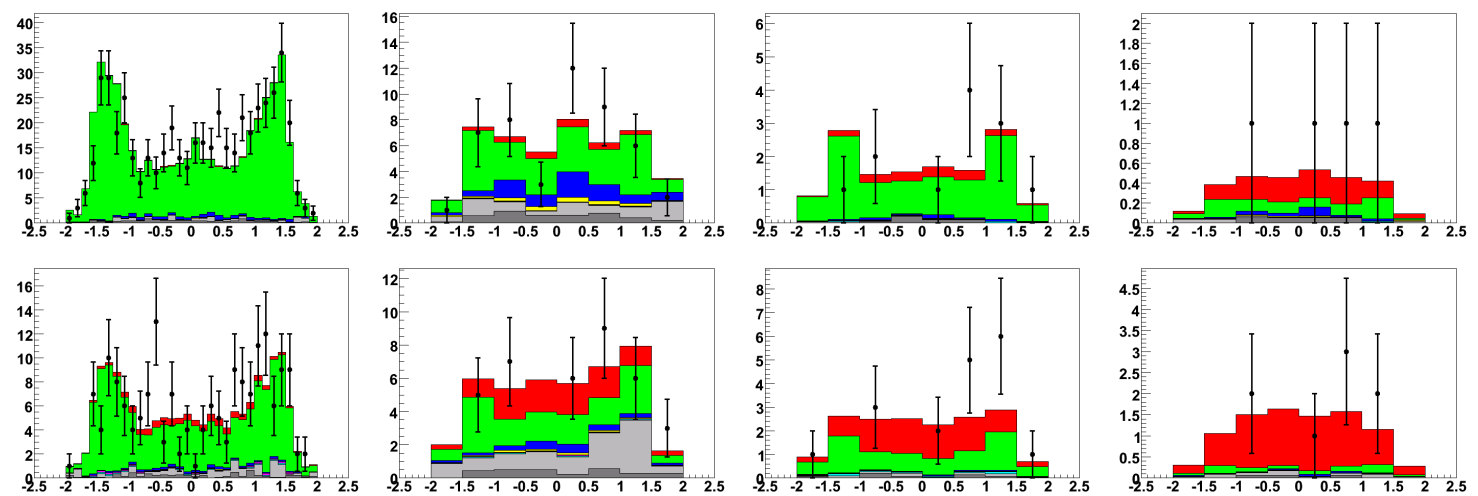

Figure 59: Track $\eta$ in electron+track. 
No $\#_{T}$ or tagging $Z_{T}$ and $\#_{T}^{Z-f i t}$ only Tagging only $E_{T}, \#_{T}^{Z-f i t}$, and tagging
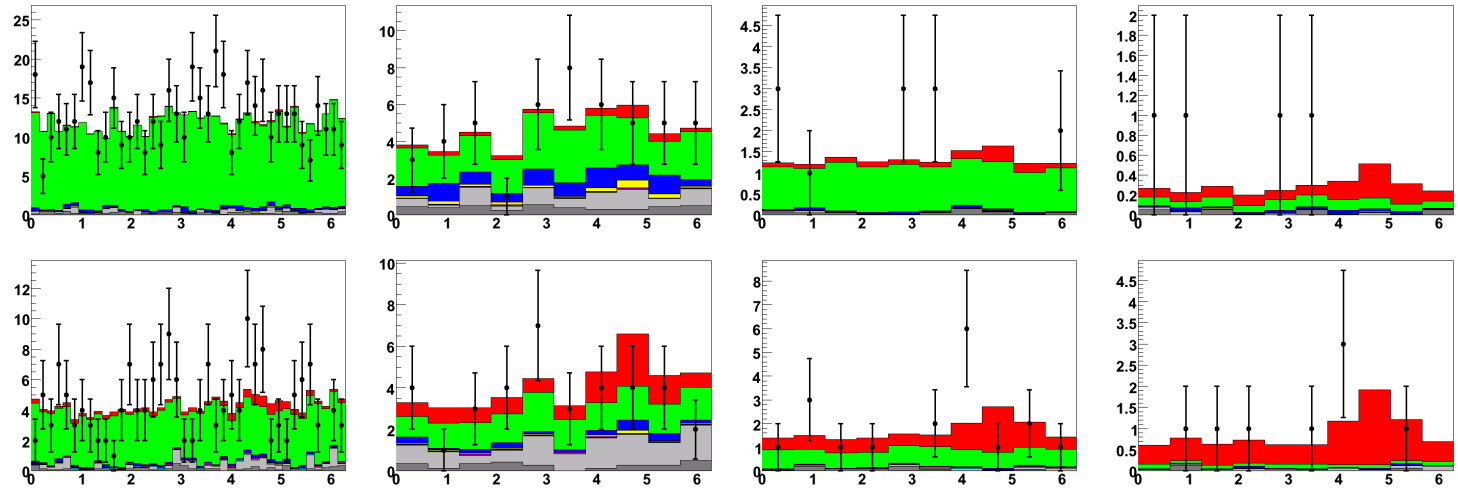

Figure 60: Track $\phi$ in electron+track.

No $Z_{T}$ or tagging $E_{T}$ and $B_{T}^{Z-f i t}$ only Tagging only $E_{T}, E_{T}^{Z-f i t}$, and tagging
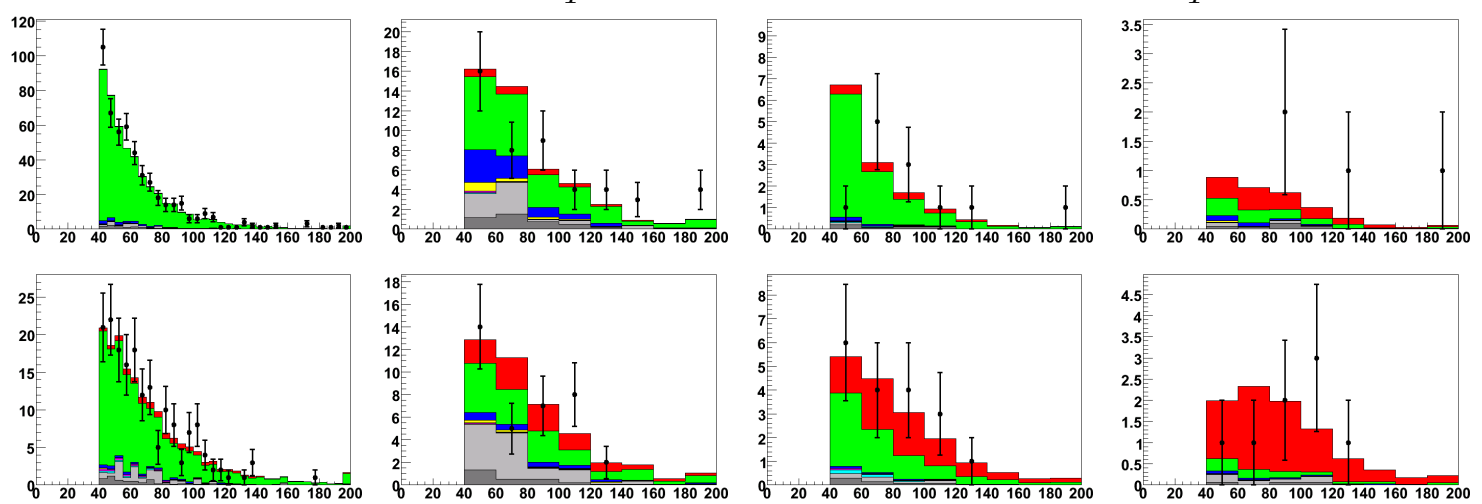

Figure 61: Leading jet $p_{T}$ (in $\mathrm{GeV}$ ) in electron+track.

No $\#_{T}$ or tagging $\#_{T}$ and $E_{T}^{Z-f i t}$ only Tagging only $\#_{T}, \#_{T}^{Z-f i t}$, and tagging
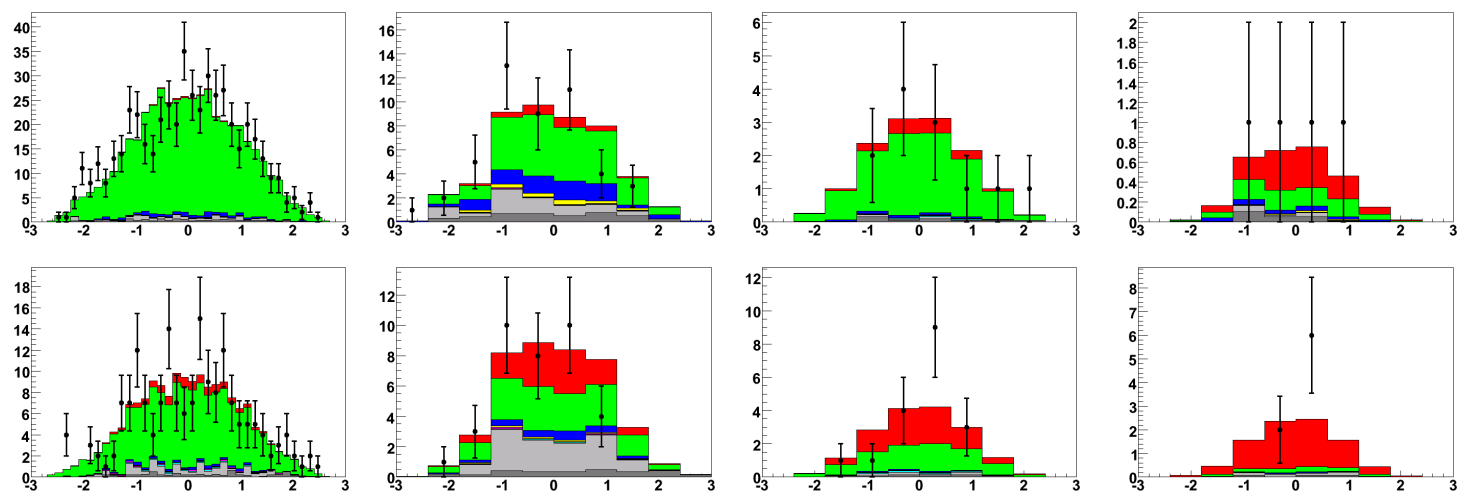

Figure 62: Leading jet $\eta$ in electron+track.

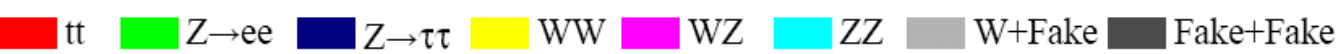


No $\#_{T}$ or tagging
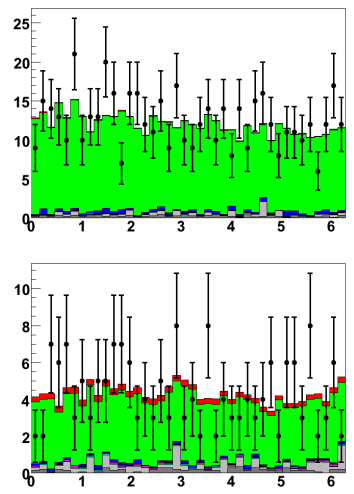

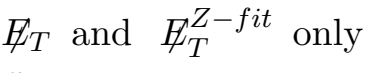
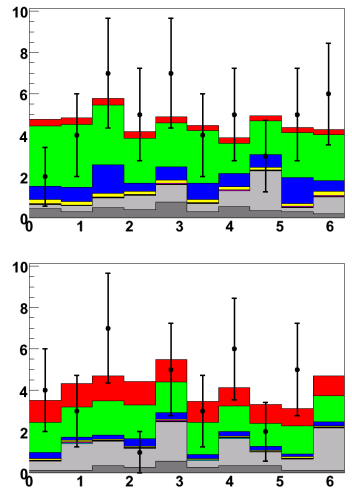

Tagging only
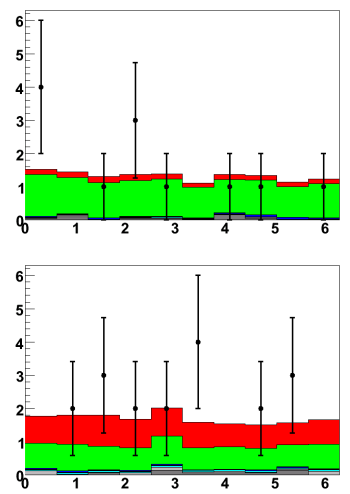

$\not_{T}, \not_{T}^{Z-f i t}$, and tagging
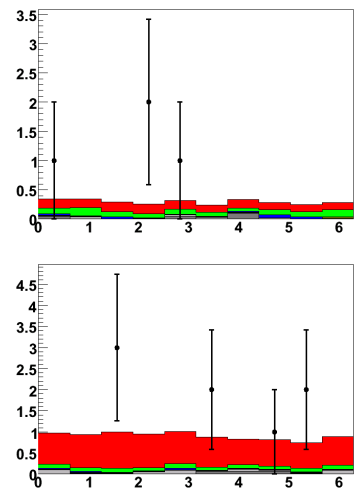

Figure 63: Leading jet $\phi$ in electron+track.

No $\not_{T}$ or tagging $\#_{T}$ and $\#_{T}^{Z-f i t}$ only Tagging only $\#_{T}, E_{T}^{Z-f i t}$, and tagging
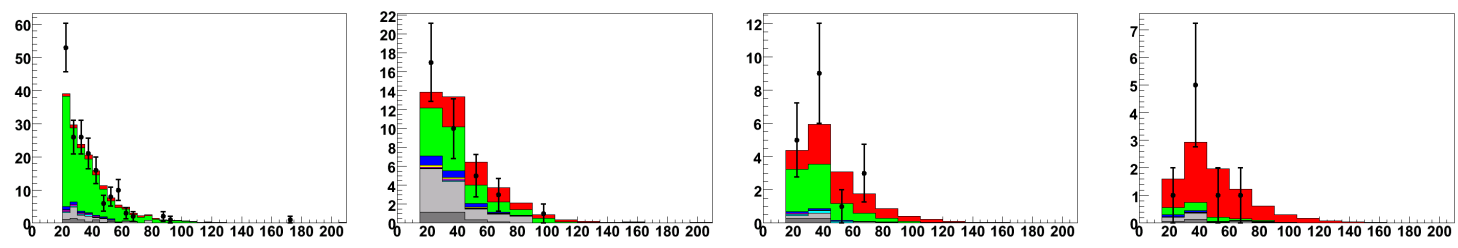

Figure 64: Second leading jet $p_{T}$ (in $\mathrm{GeV}$ ) in electron+track.

No $\#_{T}$ or tagging $\#_{T}$ and $\#_{T}^{Z-f i t}$ only Tagging only $\#_{T}, \#_{T}^{Z-f i t}$, and tagging
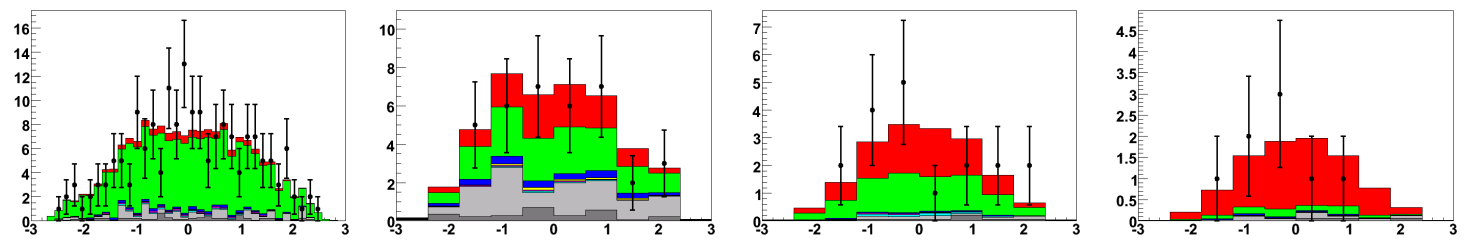

Figure 65: Second leading jet $\eta$ in electron+track. 
No $\#_{T}$ or tagging $\#_{T}$ and $\#_{T}^{Z-f i t}$ only Tagging only $\#_{T}, \#_{T}^{Z-f i t}$, and tagging
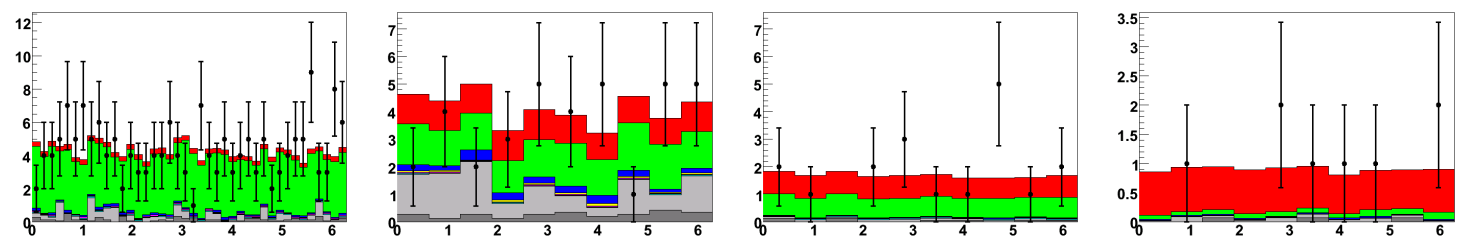

Figure 66: Second leading jet $\phi$ in electron+track.

No $\not_{T}$ or tagging $\not_{T}$ and $\#_{T}^{Z-f i t}$ only Tagging only $\not_{T}, \#_{T}^{Z-f i t}$, and tagging
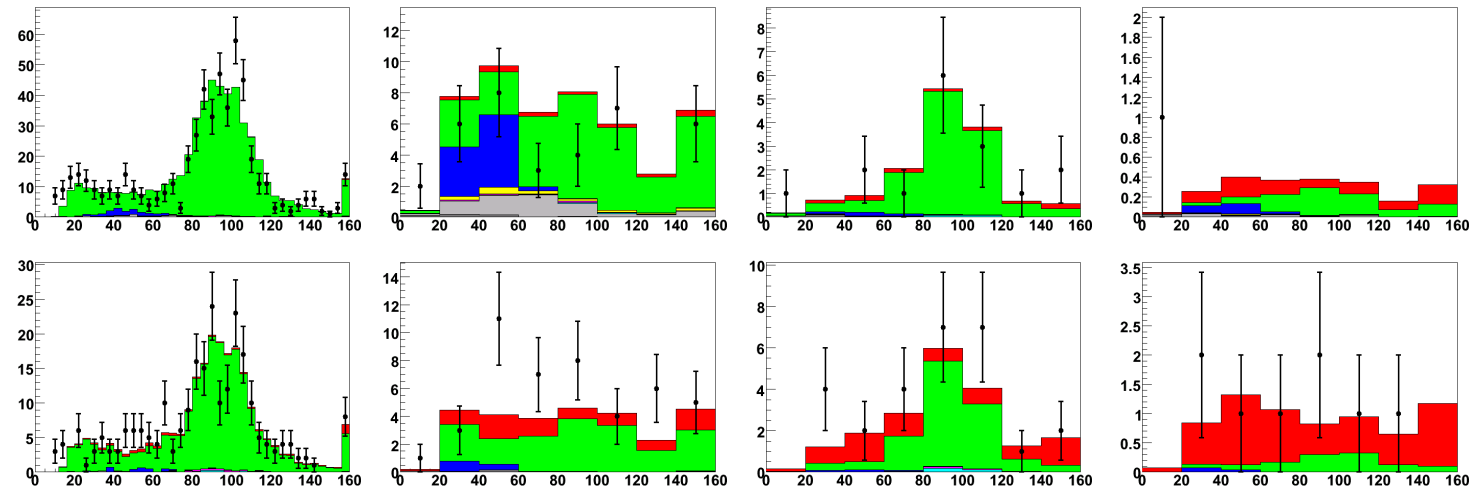

Figure 67: Mass (in GeV) of muon-track pair in muon+track.

No $\#_{T}$ or tagging $\#_{T}$ and $\#_{T}^{Z-f i t}$ only Tagging only $\#_{T}, \#_{T}^{Z-f i t}$, and tagging
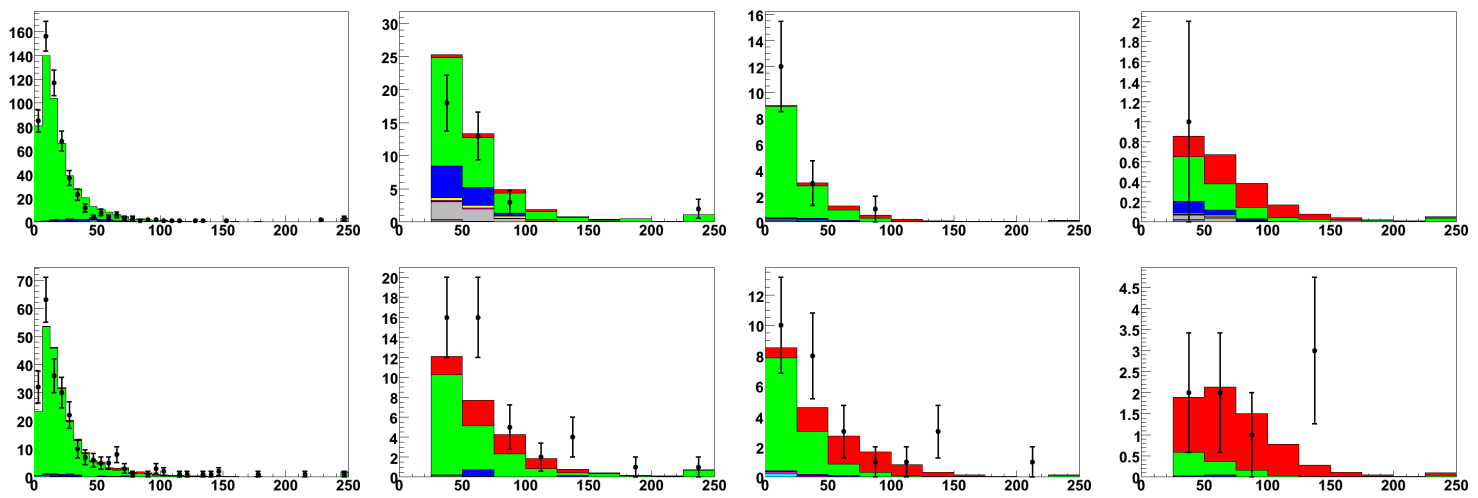

Figure 68: $\quad E_{T}($ in $\mathrm{GeV})$ in muon+track.

$\square$ tt $\quad \mathrm{Z} \rightarrow \mu \mu \square \mathrm{Z} \rightarrow \tau \tau \quad \mathrm{WW} \backsim \mathrm{WZ} \quad \mathrm{ZZ} \backsim \mathrm{W}+$ Fake $\square$ Fake+Fake 
No $\#_{T}$ or tagging $\#_{T}$ and $\#_{T}^{Z-f i t}$ only Tagging only $\#_{T}, \#_{T}^{Z-f i t}$, and tagging
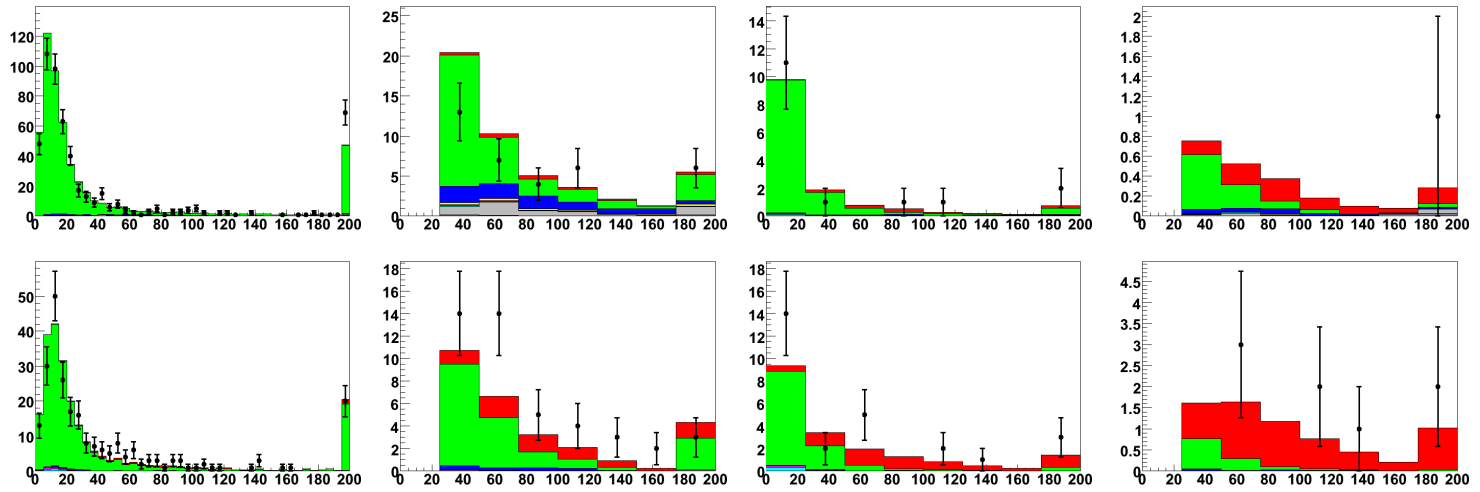

Figure 69: $\quad E_{T}^{Z-f i t}($ in $\mathrm{GeV})$ in muon+track.

No $\not_{T}$ or tagging $Z_{T}$ and $E_{T}^{Z-f i t}$ only Tagging only $Z_{T}, E_{T}^{Z-f i t}$, and tagging
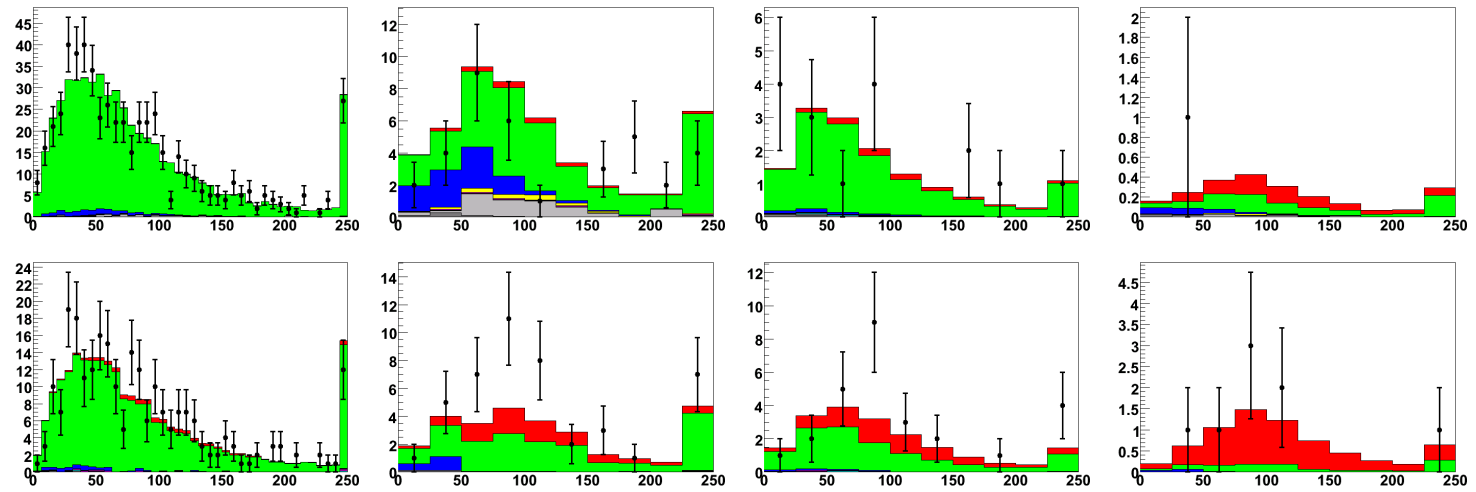

Figure 70: Transverse mass (in $\mathrm{GeV}$ ) of muon and $E_{T}$ in muon+track.

No $\#_{T}$ or tagging $\#_{T}$ and $E_{T}^{Z-f i t}$ only Tagging only $\#_{T}, \#_{T}^{Z-f i t}$, and tagging
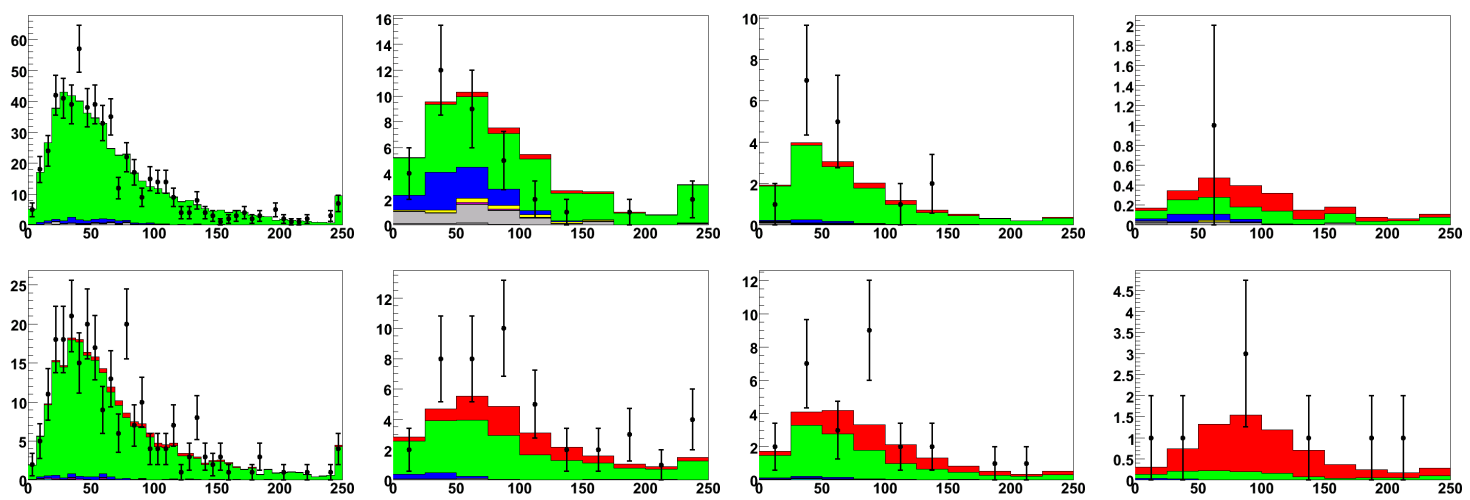

Figure 71: Transverse mass (in $\mathrm{GeV}$ ) of track and $\not_{T}$ in muon+track.

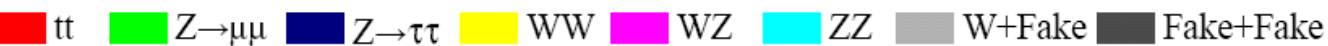


No $\#_{T}$ or tagging $Z_{T}$ and $E_{T}^{Z-f i t}$ only Tagging only $E_{T}, \#_{T}^{Z-f i t}$, and tagging
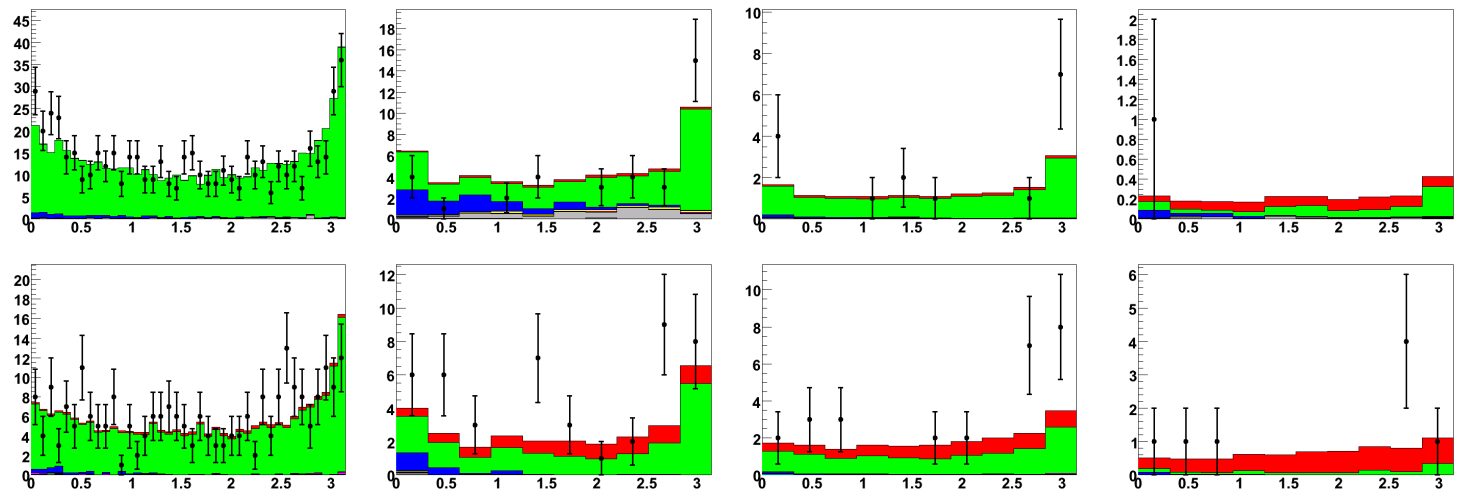

Figure 72: $\Delta \phi$ of muon and $E_{T}$ in muon+track.

No $E_{T}$ or tagging $E_{T}$ and $E_{T}^{Z-f i t}$ only Tagging only $E_{T}, E_{T}^{Z-f i t}$, and tagging
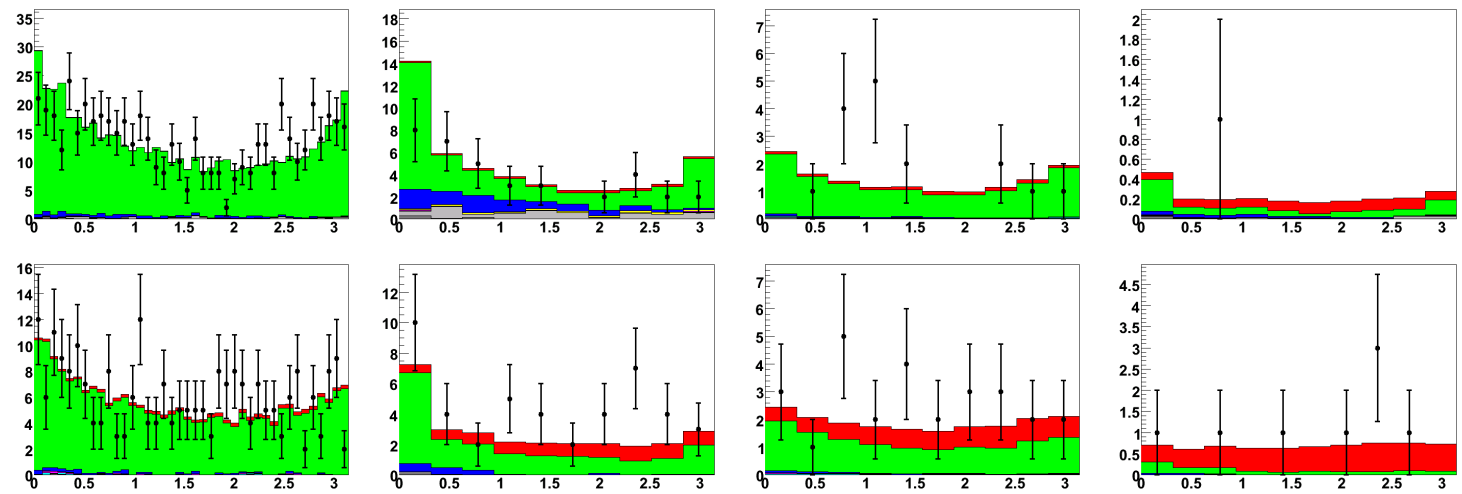

Figure 73: $\Delta \phi$ of track and $\not_{T}$ in muon+track.

No $\#_{T}$ or tagging $\#_{T}$ and $E_{T}^{Z-f i t}$ only Tagging only $E_{T}, \#_{T}^{Z-f i t}$, and tagging
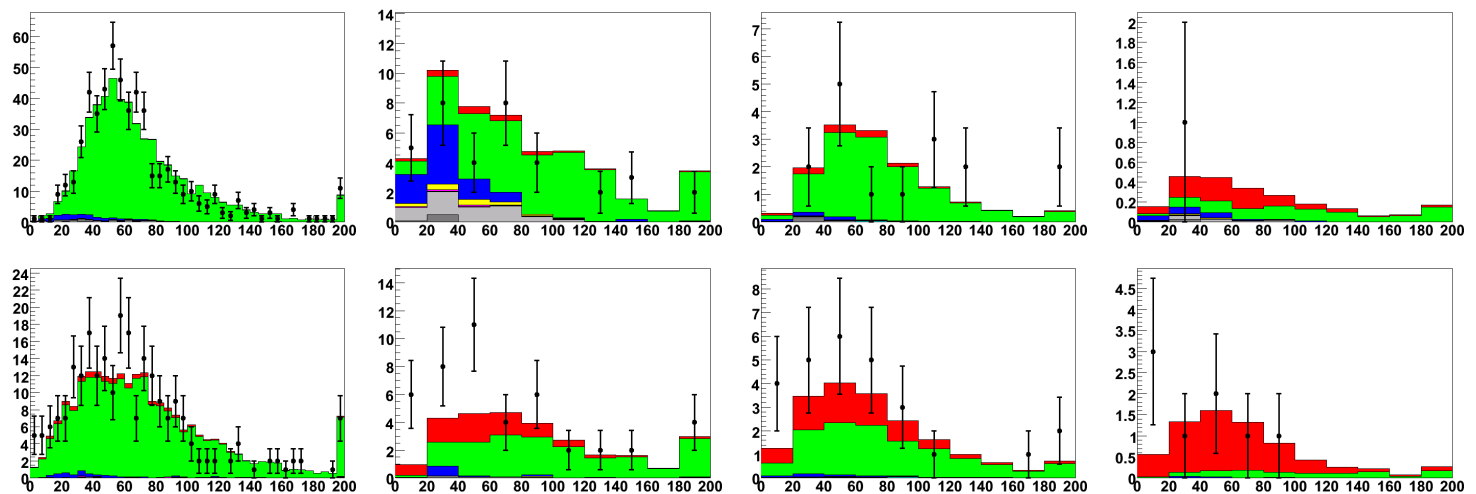

Figure 74: $\mathrm{Z} p_{T}$ (vector sum of muon $p_{T}$ and track $p_{T}$, in $\mathrm{GeV}$ ) in muon+track.

\section{$\square$ tt $\quad \mathrm{Z} \rightarrow \mu \mu \square \mathrm{Z} \rightarrow \tau \tau \quad$ WW $\square$ WZ $\quad$ ZZ $\quad$ W+Fake $\square$ Fake+Fake}


No $\#_{T}$ or tagging $Z_{T}$ and $\#_{T}^{Z-f i t}$ only Tagging only $E_{T}, \#_{T}^{Z-f i t}$, and tagging
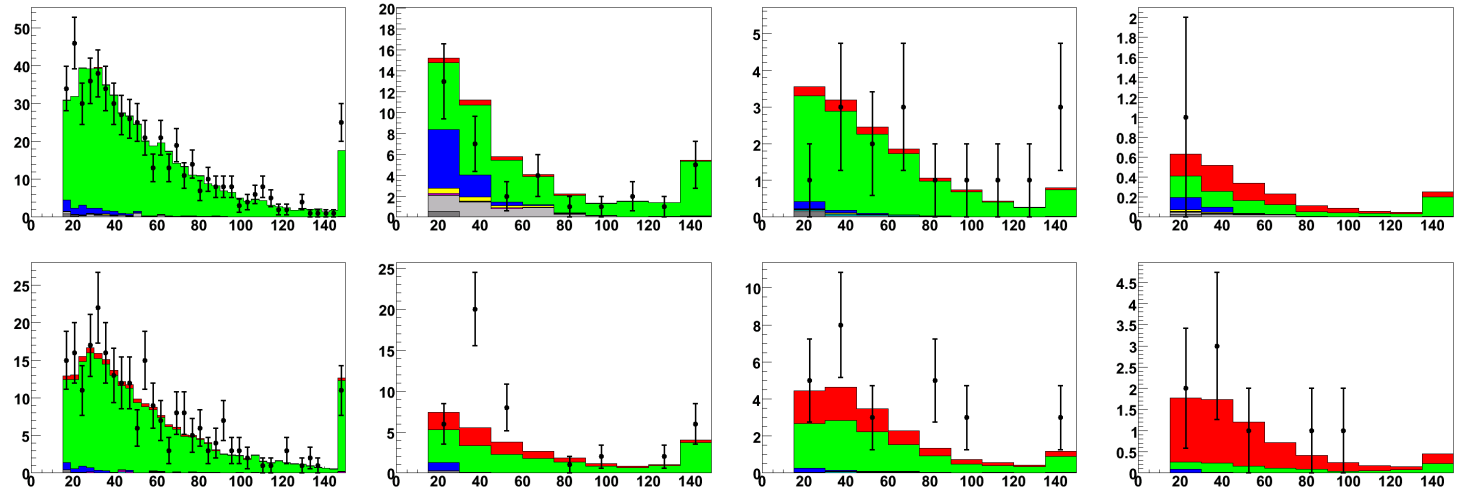

Figure 75: Muon $p_{T}$ (in $\mathrm{GeV}$ ) in muon+track.

No $E_{T}$ or tagging $\#_{T}$ and $E_{T}^{Z-f i t}$ only Tagging only $E_{T}, E_{T}^{Z-f i t}$, and tagring
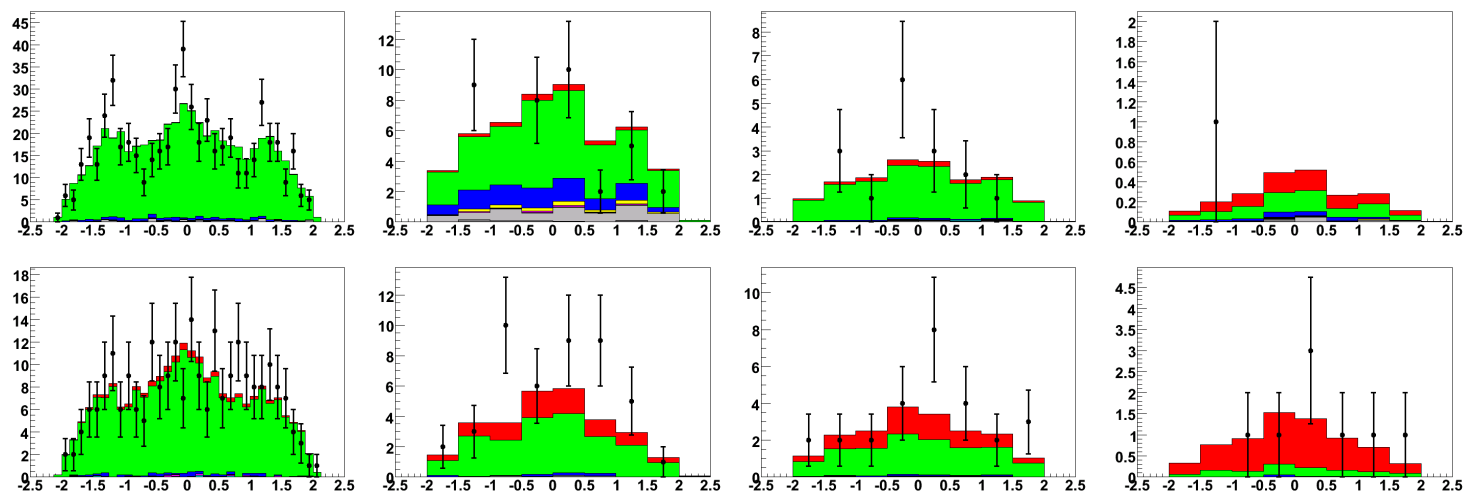

Figure 76: Muon $\eta$ in muon+track.

No $\#_{T}$ or tagging $\#_{T}$ and $E_{T}^{Z-f i t}$ only Tagging only $\#_{T}, Z_{T}^{Z-f i t}$, and tagging
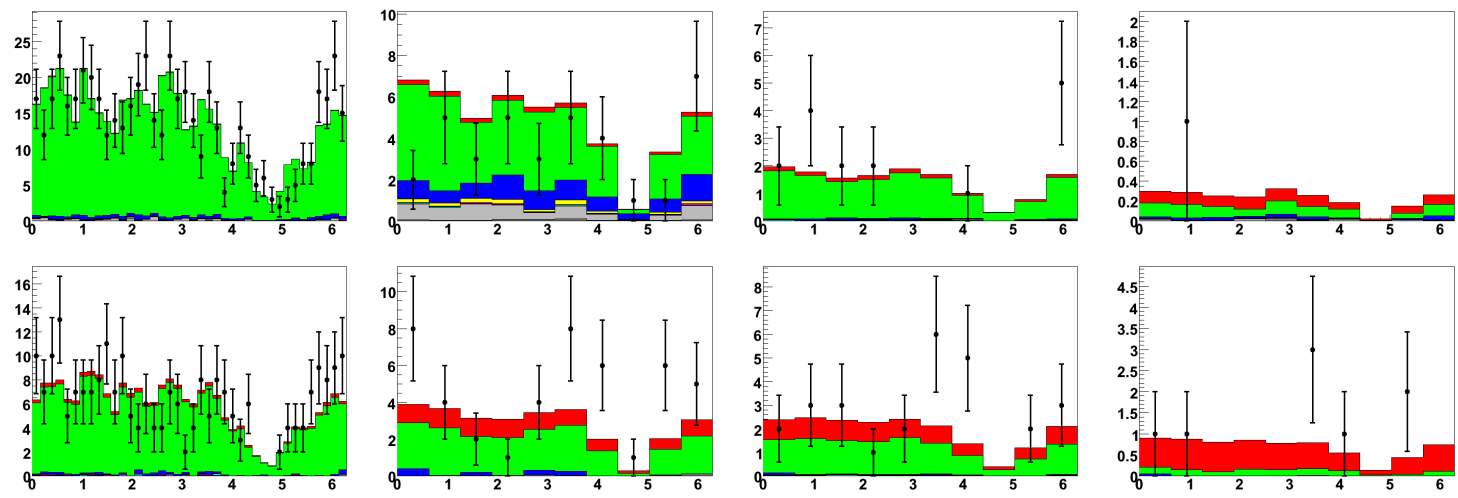

Figure 77: Muon $\phi$ in muon+track.

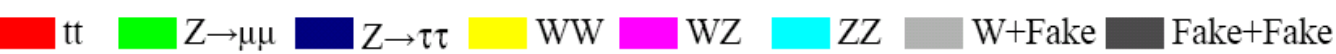


No $\#_{T}$ or tagging $Z_{T}$ and $\#_{T}^{Z-f i t}$ only Tagging only $E_{T}, \#_{T}^{Z-f i t}$, and tagging
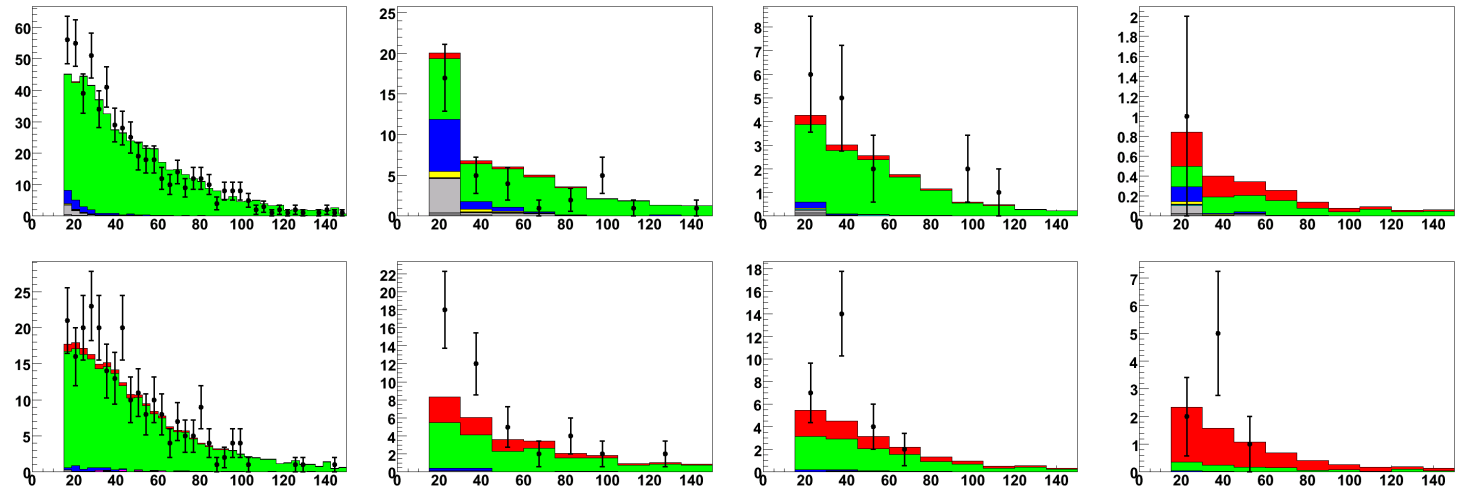

Figure 78: Track $p_{T}$ (in $\mathrm{GeV}$ ) in muon+track.

No $E_{T}$ or tagging $\not_{T}$ and $E_{T}^{Z-f i t}$ only Tagging only $E_{T}, E_{T}^{Z-f i t}$, and tagoing
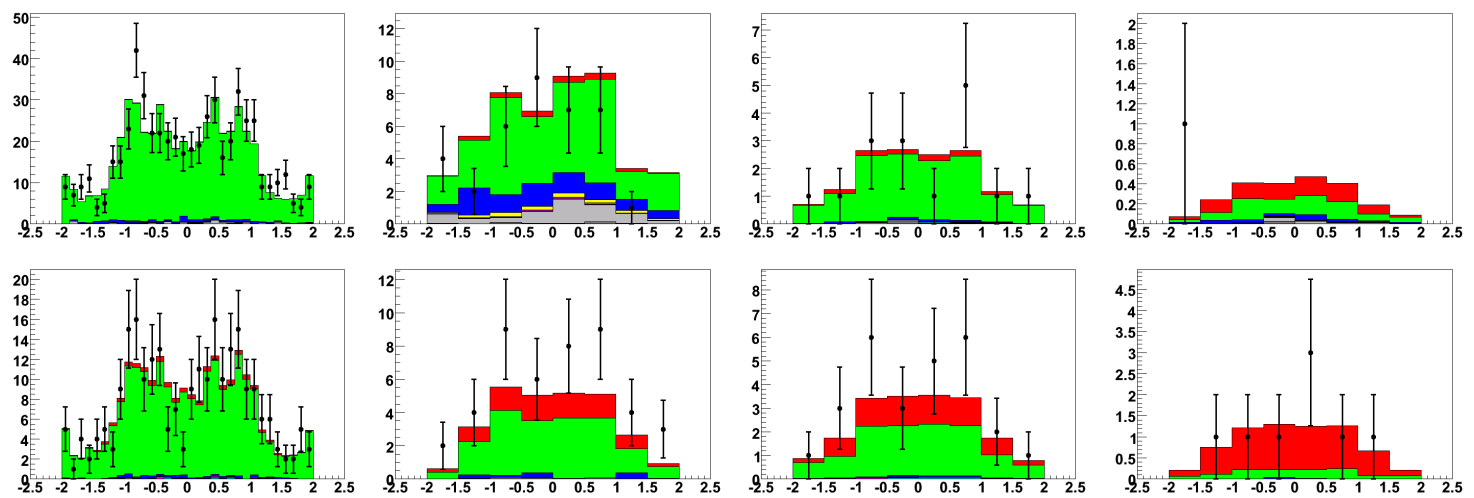

Figure 79: Track $\eta$ in muon+track.

No $\#_{T}$ or tagging $\#_{T}$ and $E_{T}^{Z-f i t}$ only Tagging only $\#_{T}, E_{T}^{Z-f i t}$, and tagging
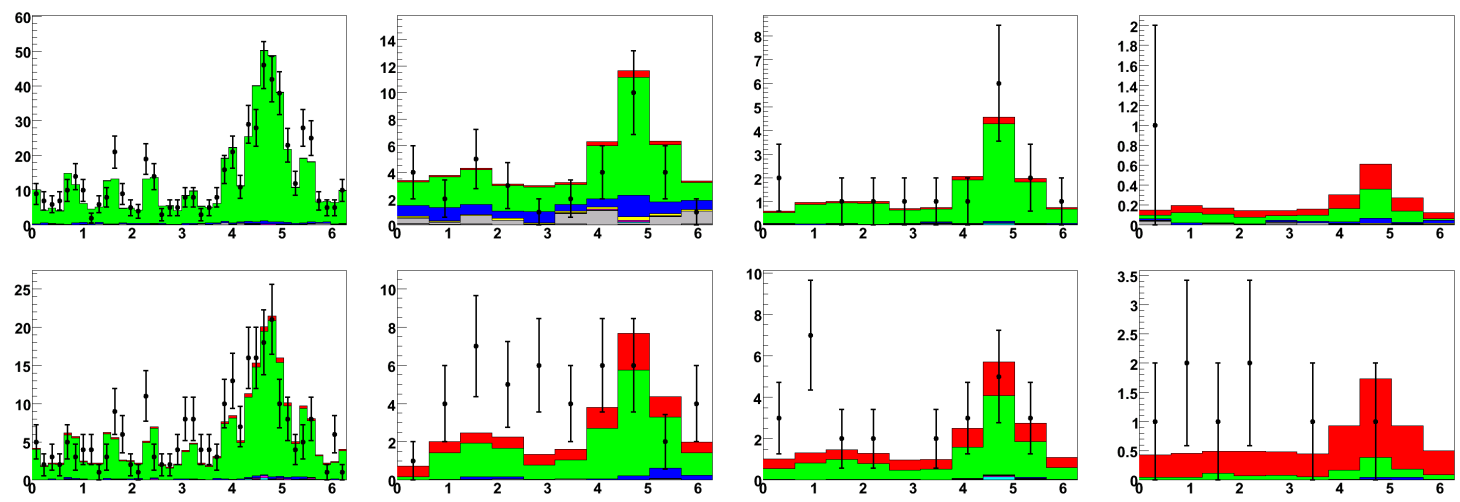

Figure 80: Track $\phi$ in muon+track.

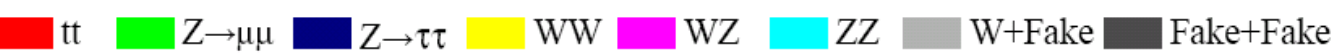


No $\#_{T}$ or tagging $\#_{T}$ and $E_{T}^{Z-f i t}$ only Tagging only $\#_{T}, \#_{T}^{Z-f i t}$, and tagging
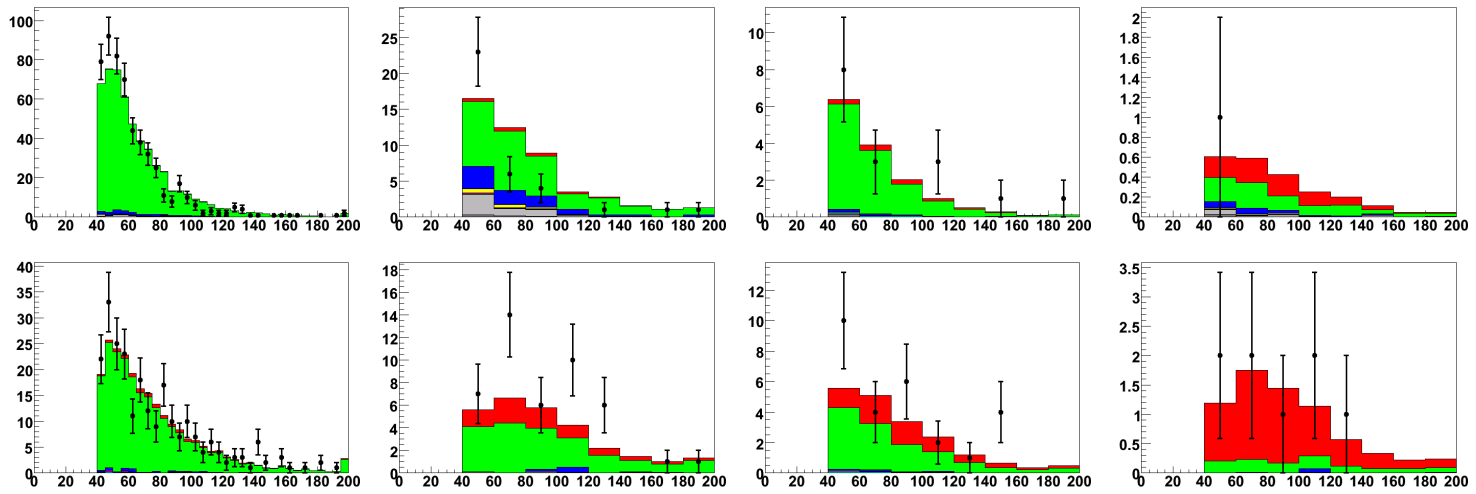

Figure 81: Leading jet $p_{T}$ (in $\mathrm{GeV}$ ) in muon+track.

No $\#_{T}$ or tagging $\not_{T}$ and $\not_{T}^{Z-f i t}$ only Tagging only $\#_{T}, E_{T}^{Z-f i t}$, and tagging
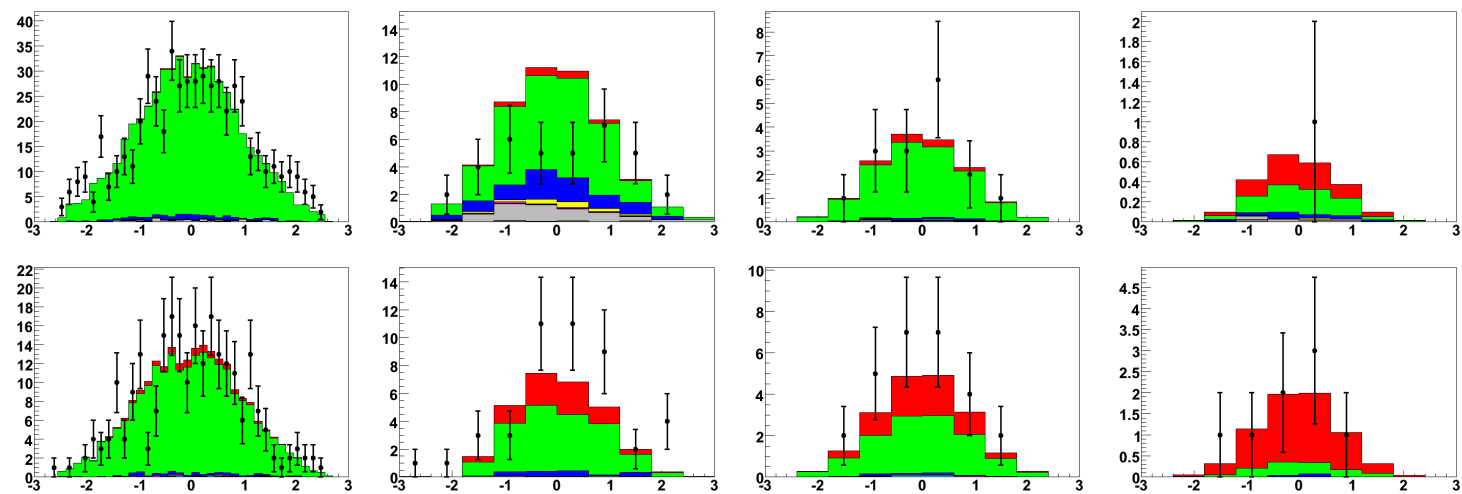

Figure 82: Leading jet $\eta$ in muon+track.

No $\#_{T}$ or tagging $E_{T}$ and $E_{T}^{Z-f i t}$ only Tagging only $E_{T}, E_{T}^{Z-f i t}$, and tagging
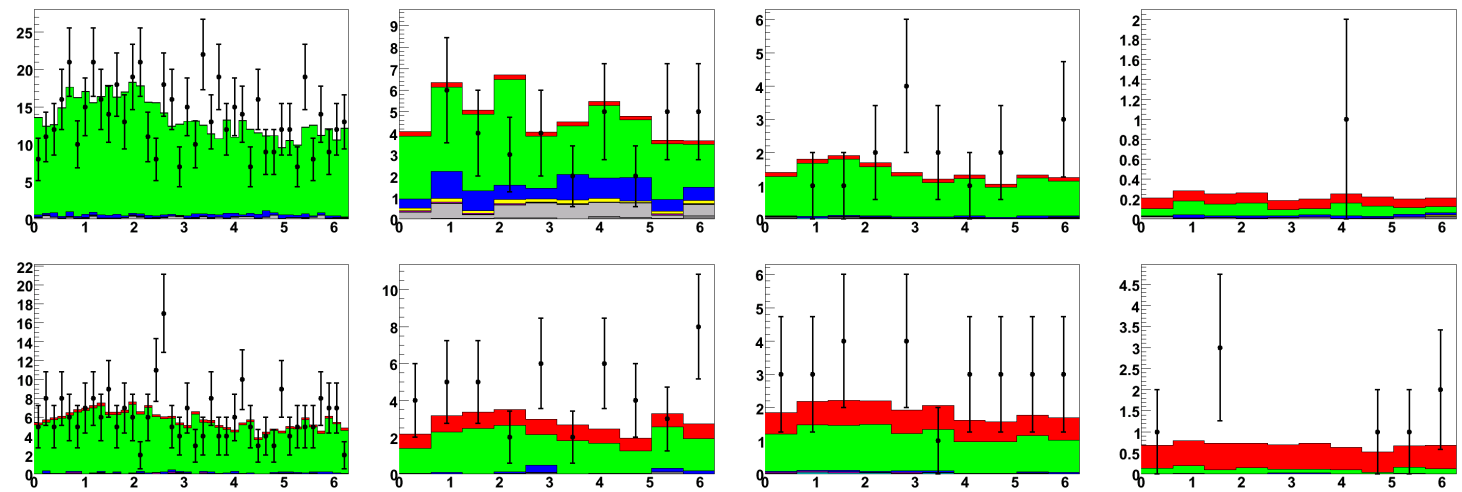

Figure 83: Leading jet $\phi$ in muon+track.

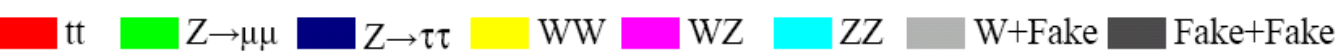


No $Z_{T}$ or tagging $\not_{T}$ and $B_{T}^{Z-f i t}$ only Tagging only $Z_{T}, E_{T}^{Z-f i t}$, and tagging
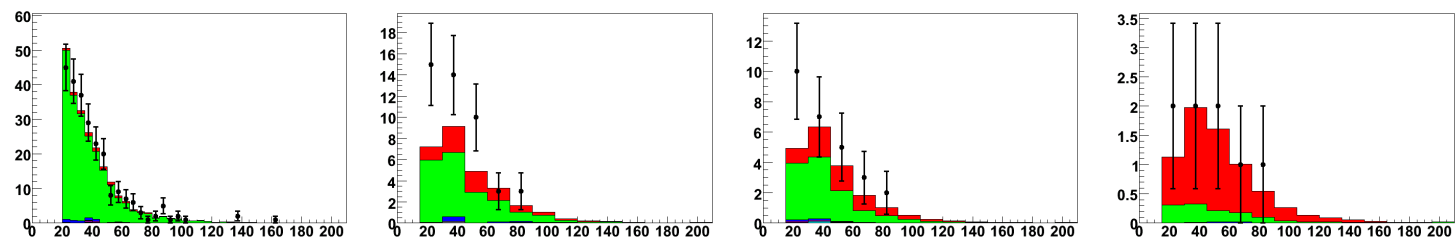

Figure 84: Second leading jet $p_{T}$ (in $\mathrm{GeV}$ ) in muon+track.

No $\#_{T}$ or tagging $\#_{T}$ and $\#_{T}^{Z-f i t}$ only Tagging only $\#_{T}, \#_{T}^{Z-f i t}$, and tagging
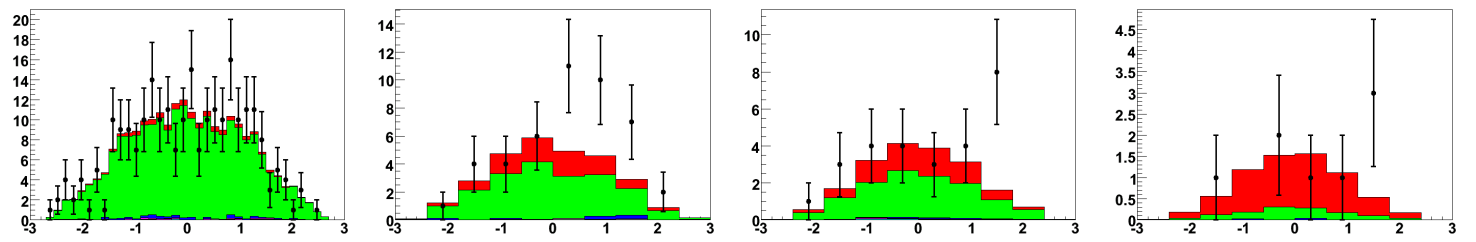

Figure 85: Second leading jet $\eta$ in muon+track.

No $\not_{T}$ or tagging $\not_{T}$ and $Z_{T}^{Z-f i t}$ only Tagging only

$\#_{T}, B_{T}^{Z-f i t}$, and tagging
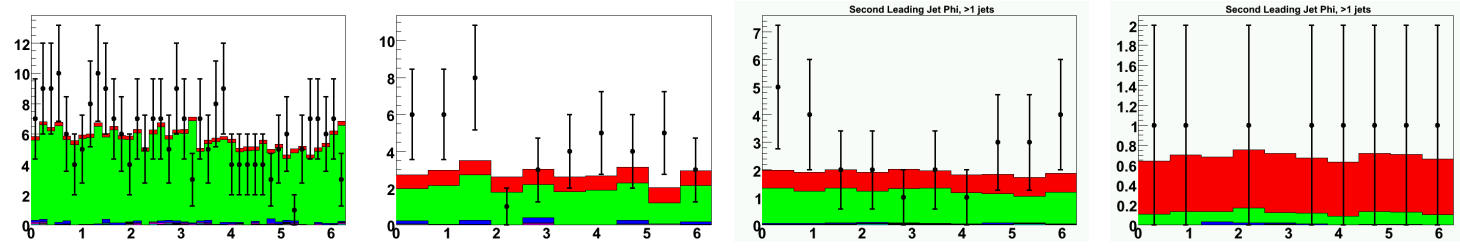

Figure 86: Second leading jet $\phi$ in muon+track. 


\section{References}

[1] The DØ Collaboration, Measurement of the $\bar{t}$ Production Cross-Section at $\sqrt{s}$ = 1.96 TeV in Dilepton Final States, DØ Note 5371-CONF, March 2007.

[2] The CDF Collaboration, Phys. Rev. Lett. 74, 2626 (1995).

[3] The DØ Collaboration, Phys. Rev. Lett. 74, 2632 (1995).

[4] C. Hill and S. Parke, Phys. Rev. D 49, 4454 (1994).

[5] N. Kidonakis and R. Vogt, Phys. Rev. D 68, 114014 (2003).

[6] T. Stelzer, Z. Sullivan and S. Willenbrock, Phys Rev D 56, 5919 (1997).

[7] B.W. Harris, E. Laenen, L. Phaf, Z. Sullivan and S. Weinzierl, Phys. Rev. D 66, 054024 (2002).

[8] S. Abachi et al., Nucl. Inst. Meth. A 338, 2422 (1995).

[9] Leon M. Lederman, Scientific American, 264(3), 48 (1991).

[10] Helen T. Edwards, Ann. Rev. Nucl. Part. Sci., 35, 605 (1985).

[11] FNAL, Design Report Tevatron I projects, Fermilab Technical Report FERMILAB-DESIGN-1984-01 (1984).

[12] FNAL, A Report of the Design of the Fermi National Accelerator Laboratory Superconducting Accelerator, Fermilab Technical (1979).

[13] Joey Thompson, Introduction to Colliding Beams at Fermilab, Fermilab Technical report Fermilab TM-1909 (1994).

[14] C.W. Schmidt and C.D. Curtis, A 50 mA Negative Hydrogen-Ion Source, IEEE Transactions on Nuclear Science, NS-26, 4120 (1979). 
[15] C.D. Curtis et al., Linac H-Beam Operation and Uses at FermiLab, IEEE Transactions on Nuclear Science, NS-26, 3760 (1979).

[16] E.L. Hubbard et al., Booster synchrotron, Fermilab Technical report Fermilab TM-405 (1973).

[17] C.S. Mishra, The Fermilab Main Injector, Fermilab Technical report Fermilab Conf-92/372 (1992).

[18] S. Nagaitsev et al., Antiproton Cooling in the Fermilab Recycler Ring, Fermilab Technical report Fermilab-Conf-05-550-AD (2005).

[19] The DØ Collaboration, The Upgraded DØ Detector, Nucl. Inst. Meth. A 565, 463-537 (2006).

[20] The DØ Collaboration, The DØ Upgrade: The Detector and its Physics Fermilab Technical Report Fermilab-Pub-96/357-E.

[21] M. Roco, The Silicon Microstrip Tracker for the D0 Upgrade, DØ Note 3553.

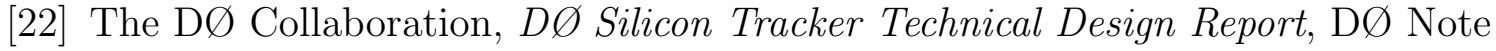
2169 (1994).

[23] The DØ Collaboration, The DØ Upgrade Central Fiber Tracker, Fermilab Technical report (1999).

[24] The DØ Collaboration, Conceptual Design of a 2 Tesla Superconducting Solenoid for the Fermilab DØ Detector Upgrade, Fermilab Technical report FERMILAB-TM-1886 (1994).

[25] Particle Data Group, Review of Particle Physics, Particle Physics B, 592, 246 (2004). 
[26] Paul Telford, An Indirect Measurement of the Width of the W Boson at the DØ Experiment, Ph.D. Thesis, University of Manchester (2006).

[27] S. Abachi et al., The DØ Detector, Nucl. Inst. Meth. A 338, 185 (1994).

[28] S. J. Wimpenny et al., The Hadron And Electron Response Of The Uranium / Liquid Argon Caorimeter Modules For The Do Detector, Nucl. Inst. Meth. A 279, 107 (1989).

[29] M. Abolins et al., Hadron and Electron Response of Uranium Liquid Argon Calorimeter Modules for the D0 Detector, Nucl. Inst. Meth. A 280, 36 (1989).

[30] The DØ Collaboration, Beam Tests of the DØ Uranium Liquid Argon End Cap Calorimeters, Nucl. Inst. and Meth. A 324, 53 (1993).

[31] B. Abbott et al., Studies of $W W$ and $W Z$ production and limits on anomalous $W W \gamma$ and $W W Z$ couplings, Phys. Rev. D 60, 072002 (1999).

[32] G.C. Blazey for the DØ Collaboration, The DØ Run II Trigger, Fermilab-Conf-97-395-E, Nov 1997.

[33] S. Youssef, Comp. Phys. Comm., 45:423-426 (1991).

[34] J. Kozminski et al., Electron Likelihood in P14, DØ Note 4449 (2004).

[35] C. Gerber et al, Muon Momentum Determination, DØ note 2140 (1994).

[36] G. Steinbruk, Measurement of the Angular Distribution of Electrons from $W$ boson decays at DØ, Ph.D. Thesis, University of Oklahoma (1999).

[37] G. Alvarez, Beauty at DØ, Ph.D. Thesis, Indiana University (1996).

[38] C. Luo, Muon Reconstruction and B Physics Studies at the Tevatron Collider Experiment DØ, Ph.D. Thesis, Indiana University (2003). 
[39] O. Peters, Muon Segment Reconstruction: A Linked List Algorithm, DØ Note 3901 (2001).

[40] Joseph G. Haley and Reinhard Schwienhorst, Level 2 EM Trigger Update for Run 2b: L2 EM Likelihood, DØ Note 5163.

[41] Particle Data Group, pdg.lbl.gov.

[42] S. Abachi et al, Top Quark Search with the DØ 1992-1993 Data Sample, Phys. Rev. D 52, 4877-4919 (1995).

[43] L. Chevalier et. al., Track Parameter Error Matrix Propagation in Matter and Magnetic Fields, Error Matricies Combination, DØ Preliminary (2001).

[44] R. Illingsworth, Development of Trigger Software for the Silicon and Fiber Trackers and a Study of B Meson Lifetimes for the DØ Experiment, Ph.D Thesis, Imperial College, London (2002).

[45] O. Boeriu, M. Hildreth, S. Jabeen, CFT Light Yield Studies, DØ Note 4602, (2004).

[46] A. Khanov, HTF: Histograming Method for Finding Tracks: The algorithm Description, DØ Note 3778 (2000).

[47] G. Borrisov, Technical Details of AA Tracking, technical talk, http://www-d0.fnal.gov/atwork/adm/d0_private/2003-02-28/adm_talk.ps (2003).

[48] G. Blazey et. al., Run II Jet Physics, DØ Note 3750 (2000).

[49] G. Davis, First Measurement of the Differential Inclusive Cross Section for Jet Production at DØ Run II, Ph.D Thesis, University of Rochester (2004). 
[50] DØ Jet Energy Scale Group, Jet Energy Scale (CAFIX 5.1), DØ note 3139 (1996).

[51] Elvira, D., Jet Energy Scale at DØ, DØ Note 3287 (2001).

[52] U. Basssler and G. Bernardi, Towards a Coherent Treatment of Calorimetric Energies: Missing Transverse Energy, Jets, E.M. Objects and the T42 Algorithm, DØ Note 4124 (2003).

[53] M. Angelou, DØ Top Analysis and Data Sample for the Winter Conference 2004, DØ Note 4419 (2004).

[54] B. Abbott et al, Determination of the Absolute Jet Energy Scale in the DØ Calorimeters, Nucl. Inst. Meth. A 424, 352-394 (1999).

[55] F. Hsieh et al, Jet Energy Scale Uncertainty for Top Cross Section PRL, DØ note 3200 (1997).

[56] F. Hsieh and H. Lan, Post-CAFIX Jet Corrections For Top Mass Analyses, DØ Note 3055, (1996).

[57] F. Hsieh, Study of the Jet Energy Scale Using Gamma + 1 jet Events, DØ Note 3130, (1996).

[58] B. Abbott et al., Direct Measurement of the Top Quark Mass by the DØ Collaboration, Phys. Rev. D 58, 52001 (1998).

[59] A. Schwartzman and M. Narain, Probabilistic Primary Vertex Reconstruction, DØ Note 4025 (2004).

[60] M. Anastasoaie, S. Robinson and T. Scanlon, Performance of the NN b-tagging Tool on p17 Data, DØ Note 5213. 
[61] http://www-clued0.fnal.gov/\%7Enunne/cross-sections/mcfm_crosssections.html

[62] V. Abazov et al., Measurement of the tt Production Cross Section in pp Collisions at $\sqrt{(s)}=1.96$ TeV Using Secondary Vertex b Tagging, Phys. Rev. D 74, $112004(2006)$.

[63] M.L. Mangano et al., ALPGEN, a Generator for Hard Multiparton Processes in Hadronic Collisions, JHEP 307, 001 (2003), hep-ph/0206293.

[64] T. Sjojstrand et al., High-Energy-Physics Event Generation with PYTHIA 6.1, Comp. Phys. Commun. 135, 238 (2001), hep-ph/0010017;

[65] M. Arthaud et al., Measurement of the tt Production Cross-section at $\sqrt{s}=$ $1.96 \mathrm{TeV}$ in Electron Muon Final States Using p17 Data Set, DØ Note 5360.

[66] Y. Peters et al., Study of the W+Jets Heavy Flavor Scale Factor in p17, DØ Note 5406.

[67] S.J. Park and M. Begel, Efficiency of the Data Quality Calorimeter Flags, DØ Note 5324.

[68] B. Martin dit Latour, Y. Arnoud, G. Sajot, Measurement of the tt Cross-section at $\sqrt{s}=1.96 \mathrm{TeV}$ in ee Final States Using 17 Data Set, draft of $\mathrm{D} \varnothing$ Note available at http://www-clued0.fnal.gov/ ${ }^{\sim}$ sajot/top_ee_cross_section_note_v1.pdf

[69] T. Gadfort et al., Muon Identification Certification for p17 Data, D $\varnothing$ Note 5157.

[70] T. Andeen et al., The DØ Experiment's Integrated Luminosity for Tevatron Run IIa, Fermilab Technical Report FERMILAB-TM-2365 (2007). 
[71] The D $\varnothing$ Collaboration, Combined tt Production Cross Section in the Lepton+Track and Dilepton Final States using $1 \mathrm{fb}^{-1}$, DØ Note 5477-CONF, August 2007.

[72] E.W. Varnes, Track Reconstruction Efficiency Measurement with Single Muons, DØ Note 4317.

[73] J. Hays et al., Single Electron Efficiencies in 177 Data and Monte-Carlo Using p18.05.00 d0correct, DØ Note 5105.

[74] P. Calfayan et al., Muon Identification Certification for p17 Data, D $\varnothing$ Note 5157.

[75] The DØ Collaboration, Phys. Rev. Lett. 100, 192004 (2008). 